\title{
Desenvolvimento e Análise de Estruturas de Dependência via Cópulas
}

\author{
ULISSES UMBELINO DOS ANJOS
}

Tese apresentada

ao

Instituto de Matemática e Estatística

da

Universidade de São Paulo

para

a obtenção do grau

de

Doutor em Estatística.

Área de Concentração: Probabilidade e Estatística Aplicada

Orientador: Nikolai Valtchev Kolev

São Paulo - Junho de 2005

${ }^{\dagger}$ Este trabalho contou com apoio financeiro da CNPq - Proc. $\mathrm{N}^{\mathrm{O}} 141503 / 2002-5$ 


\section{Desenvolvimento e Análise de Estruturas de Dependência via Cópulas}

Este exemplar corresponde à redação final da tese devidamente corrigida e defendida por Ulisses Umbelino dos Anjos e aprovada pela comissão julgadora.

São Paulo, 13 de Junho de 2005.

Banco Examinadora:

- Prof. Dr. Nikolai Valtchev Kolev (Orientador) - IME - USP

- Prof. Dr. Nelson Ithiro Tanaka - IME - USP

- Profa. Dra. Beatriz Vaz de Melo Mendes - Docente externo

- Prof. Dr. Renato Martins Assunção - Docente externo

- Prof. Dr. Álvaro de Lima Veiga Filho - Docente externo 
A Deus pela vida e oportunidade de fazer este doutorado aqui no IME-USP; Aos meus pais pelo amor, carinho e apoio dedicados todos estes anos; À minha esposa e filho pelo carinho, amor e compreensão;

Aos meus irmãos que embora distantes sempre torceram por mim; Que Deus abençoe muito as vidas de todos vocês.

Bem aventurado aquele que teme ao Senhor e anda nos seus caminhos. Sl 128:1 


\section{Agradecimentos}

Ao meu orientador Prof. Nikolai que participou ativamente deste trabalho proporcionando-me muitas oportunidades de aprendizagem e troca de experièncias com pesquisadores de nível internacional, além ter sido um grande companheiro nesta jornada.

Ao Prof. Nelson Tanaka pelos seus preciosos comentários e contribuições.

Ao Prof. Roger Nelsen que gentilmente leu parte deste trabalho e fez valiosas observaçòes .

Aos meus amigos e colegas aqui do IME, em particular ao Alberto, Elmo, Iran e William que foram e são grandes companheiros.

Ao CNPq pelo apoio financeiro, sem a qual este projeto não seria possível. 


\section{Resumo}

Neste trabalho apresentamos vários resultados relacionados com a teoria de cópulas. É feita uma representação para distribuições bivariadas que utiliza uma nova medida de dependència local que denominamos função Spearman e estudamos suas propriedades. Apresentamos a cópula associada a estrutura de dependência de estatísticas de ordem bivariadas, mostramos uma relação de recorrência assim como os limites de Fréchet associados. Finalmente, mostramos alguns resultados relacionados com a análise da dependência de vetores aleatórios não sobrepostos, apresentando uma adaptação do método de Cohen para cópulas em que deste modo pode-se construir uma $\left(n_{1}+n_{2}\right)$-dimensional cópula $C$ consistente com as cópulas $n_{1}$-dimensional cópula $C_{1}$ e $n_{2}$-dimensional cópula $C_{2}$ associadas com as marginais multivariadas dadas. Apresentamos também outra ferramenta que utiliza cópulas para estudar a estrutura de dependência de vetores aleatórios não sobrepostos em que as marginais são as distribuições de Kendall associadas aos vetores aleatórios. 


\section{Abstract}

In this work we present several developments concerned with copula theory. We propose a representation of bivariate distributions through a new local measure of dependence that we call Spearman function and study its properties. Also, we exhibit the copula associated with bivariate order statistics, give a recurrence relationship between the copulas of the order statistics as well as theirs related Fréchet bounds. Finally, in order to describe the dependence structure between nonoverlapping random vectors, we present a adaptation of Cohen method for constructing a $\left(n_{1}+n_{2}\right)$-dimensional copula $C$ consistent with given $n_{1}$-dimensional copula $C_{1}$ and $n_{2}$-dimensional copula $C_{2}$ associated with the given multivariate marginals. Also, we provide a method to use copulas to describe the dependence structure between nonoverlapping random vectors, where the marginals are Kendall distributions associated to these nonoverlapping random vectors. 


\section{Sumário}

1 Introdução

2 Preliminares 4

2.1 Transformada integral da probabilidade . . . . . . . . . . 4

2.2 Cópulas . . . . . . . . . . . . . . . . . 7

2.3 Transformada de nível . . . . . . . . . . . . . 11

$2.4 \mathrm{O}$ fenômeno da dependência $\ldots \ldots \ldots \ldots \ldots$

2.4.1 Comonotonicidade e contra-monotonicidade . . . . . . . 13

2.4 .2 Medidas de dependência . . . . . . . . . . . . 15

2.4.3 Concordância e Medidas de Concordância . . . . . . . . . . 17

3 Representação para cópulas bivariadas 21

3.1 Uma nova representação para cópulas . . . . . . . . . . . . . . . 24

3.2 Caracterização e propriedades da função Spearman . . . . . . . . . 30

3.2 .1 Caracterização da função Spearman . . . . . . . . . . . . 30

3.2 .2 Propriedades da função Spearman . . . . . . . . . . . . . . 34

3.3 Representação para cópulas via cópula gaussiana local . . . . . . . . 35

3.3 .1 Propriedades . . . . . . . . . . . . . . 37

4 Cópula para estatísticas de ordem $\quad 39$

4.1 Cópula para estatísticas de ordem . . . . . . . . . . . . 41 
4.2 Cópula Assintótica . . . . . . . . . . . . . . . . . . . 44

4.3 Relação de recorrència . . . . . . . . . . . . . . . . . 48

4.4 Fronteiras de Fréchet $\ldots \ldots \ldots \ldots$. . . . . . . . . . 50

5 Cópulas com marginais multivariadas 54

5.1 O método de Cohen para cópulas . . . . . . . . . . 58

5.2 Cópulas para vetores aleatórios não sobrepostos . . . . . . . . . 64

Conclusões

Bibliografia

$\begin{array}{lr}\text { A Programas } & 79\end{array}$

A.1 Programa Seção $4.2 \ldots \ldots$. . . . . . . . . . . . . . 79

A.2 Programa Seção $4.4 \ldots \ldots$. . . . . . . . . . . . 81 


\section{Capítulo 1}

\section{Introdução}

Nos últimos anos tem crescido na área de Finanças e Atuária o interesse por modelos que agreguem mais informações e por conseqüência aumentou também o interesse por modelos multivariados. Entretanto, alguns problemas surgem quando utilizamos tais modelos. Um deles é a escolha da estrutura de dependência mais adequada. Entender as relações entre variáveis aleatórias é um problema central na ciência Estatística. Por esse motivo a teoria de cópulas tem despertado tanto interesse pois ela fornece ferramentas que auxiliam a escolha de estruturas de dependência adequadas e métodos para a construção de novas estruturas de dependência. É crescente o número de artigos que tratam de aplicações de cópulas nos mais variados segmentos de finanças quantitativas, como por exemplo análise de risco de mercado, cálculo de risco de uma carteira de seguros, apreçamento de derivativos, veja por exemplo Cherubini et al. (2004), Embrechts et al. (2003a) e Embrechts et al. (2003b). As cópulas representam uma abordagem útil para modelar e entender o fenòmeno da dependência entre as variáveis aleatórias pois elas permitem estudar a dependência entre variáveis aleatórias sem a interferència da estrutura probabilística das marginais, visto que as cópulas são funções distri- 
buição multivariadas cujas marginais são sempre uniformes. Portanto, toda cópula é uma função distribuição conjunta, mas nem toda função distribuição conjunta é uma cópula.

O objetivo deste trabalho é desenvolver ferramentas utilizando cópulas que possam auxiliar na tarefa de escolher estruturas dependència adequadas assim como métodos que possibilitem a construção de novas estruturas de dependência.

Este trabalho está organizado da seguinte maneira:

No Capítulo 2 é feita uma exposição dos principais resultados e definições da teoria de cópulas que serão pertinentes neste trabalho.

No Capítulo 3 apresentamos uma representação para cópulas bivariadas utilizando uma medida de dependência local que denotamos por $\rho_{C}$ e chamamos de função Spearman associada à cópula $C$. Como veremos, $\rho_{C}$ é o coeficiente de correlação linear entre as variáveis indicadoras $I\{U \leq u\}$ e $I\{V \leq v\}$ para cada par $(u, v) \in[0,1]^{2}$ fixo.

Na Seção 3.3, Capítulo 3, mostramos uma outra forma de representação para cópulas que faz uso de uma medida de dependência local $r(u, v)$ e a cópula gaussiana $\Phi_{r}$ com coeficiente $r=r(u, v)$.

No Capítulo 4 exibimos a cópula $C_{X_{r: n}, Y_{s: n}}$ relacionada às estatísticas de ordem $X_{r: n}$ e $Y_{s: n}$ do vetor aleatório $(X, Y)$ com cópula associada $C$. Na Seção 4.2, apresentamos uma aproximação para a distribuição conjunta de $\left(X_{r: n}, Y_{s: n}\right)$ utilizando a estrutura de dependência assintótica das estatísticas de ordem capturada pela cópula assintótica. Na Seção 4.3 fornecemos uma relação de recorrència para as cópulas de estatística de ordem e na Seção 4.4 apresentamos suas respectivas fronteiras de Fréchet. 
No Capítulo 5 é feita uma revisão sobre os resultados que envolvem o estudo da dependência entre vetores aleatórios e em seguida na Seção 5.1 apresentamos uma adaptação do método de Cohen, veja Cohen (1984), para cópulas. Na Seção 5.2 mostramos uma nova metodologia para estudar a dependència entre vetores aleatórios não sobrepostos que utiliza a cópula das distribuições de Kendall associadas aos vetores aleatórios. A distribuição de Kendall associada ao vetor $(X, Y)$ com cópula correspondente $C$ por exemplo, é a função distribuição associada a variável aleatória $C(U, V)$. 


\section{Capítulo 2}

\section{Preliminares}

Um resultado básico na teoria de cópulas é a transformada integral da probabilidade. É esse resultado que faz o elo de ligação entre o espaço das variáveis aleatórias uniformes multivariadas e o espaço das variáveis aleatórias de interesse.

\subsection{Transformada integral da probabilidade}

Na Matemática o conceito das transformadas é bem conhecido, e.g. transformada de Laplace, de Fourier, etc. A transformada integral da probabilidade será definida de maneira similar ao das transformadas em geral, pois deste modo será mais fácil introduzir o conceito da transformada de nível que pode ser interpretada como uma extensão da mesma, veja Szczesny (1991).

Definição 2.1. A transformada integral da probabilidade da função distribuição $F$ da variável aleatória $X$ é dada por

$$
\mathcal{I}(F)(u)=\int_{-\infty}^{+\infty} I(F(x), u) d F(x)=E[I(F(X), u)], \text { para } u \in[0,1],
$$

em que

$$
I(F(x), u)= \begin{cases}0 & \text { se } F(x)>u \\ 1 & \text { se } F(x) \leq u\end{cases}
$$


Observação 2.1. A transformação $\mathcal{I}(F)(u)$ fornece a função distribuição da variável aleatória $U=F(X)$, i.e.,

$$
\mathcal{I}(F)(u)=P(U \leq u), \text { para } u \in[0,1]
$$

Observação 2.2. Para toda função distribuição $F$ contínua tem-se:

$$
\mathcal{I}(F)(u)=P(U \leq u)=u, \text { para } u \in[0,1] .
$$

Portanto se $F(x)$ for contínua, a transformação $\mathcal{I}(F)(u)$ retornará sempre a função distribuição uniforme em $[0,1]$ a ser denotada por $U(0,1)$. De fato, seja $X$ uma variável aleatória qualquer e F sua função distribuição contínua. Então, para todo $u \in[0,1]$,

$$
\begin{aligned}
\mathcal{I}(F)(u) & =\int_{-\infty}^{+\infty} I(F(x), u) d F(x) \\
& =\int_{\{x: F(x) \leq u\}} I(F(x), u) d F(x)+\underbrace{\int_{\{x: F(x)>u\}} I(F(x), u) d F(x)}_{=0} \\
& =\int_{\{x: F(x) \leq u\}} 1 d F(x) \\
& =\int_{-\infty}^{F^{-1}(u)} d F(x)=F\left(F^{-1}(u)\right)-F(-\infty)=u .
\end{aligned}
$$

Portanto, desde que a função distribuição $F$ da variável aleatória $X$ seja contínua, a transformada integral da probabilidade de $F$ sempre resultará em uma função distribuição $U(0,1)$.

Exemplo 2.1. Seja $X$ uma variável aleatória, tal que

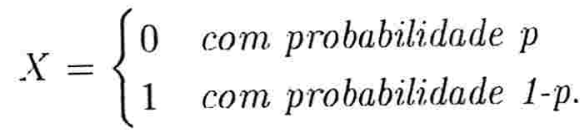


portanto,

$$
F(x)= \begin{cases}0 & \text { para } x \in(-\infty, 0) \\ p & \text { para } x \in[0,1) \\ 1 & \text { para } x \in[1, \infty)\end{cases}
$$

A transformada integral da probabilidade $\mathcal{I}(F)(u)$ é dada por,

$$
\begin{aligned}
\mathcal{I}(F)(u) & =\int_{-\infty}^{+\infty} I(F(x), u) d F(x) \\
& =p I(F(0), u)+(1-p) I(F(1), u) \\
& =p I(p, u)+(1-p) I(1, u),
\end{aligned}
$$

assim,

$$
\mathcal{I}(F)(u)=P(U \leq u)= \begin{cases}0 & \text { para } u \in[0, p) \\ p & \text { para } u \in[p, 1) \\ 1 & \text { para } u=1\end{cases}
$$

Portanto, temos uma função distribuição no intervalo $[0,1]$ mas que não é a distribuição uniforme. Desse modo, pode-se dizer que a transformada integral da probabilidade não é "fechada" no espaço das variáveis uniformes no intervalo $[0,1]$. Por outro lado, se definirmos a função inversa como segue,

$$
F^{-1}(u)=\inf \{x: F(x) \geq u\}
$$

pode-se verificar facilmente o seguinte resultado.

Lema 2.1. Para qualquer variável aleatória $X$ com função distribuição $F$ e $U \sim$ $U(0,1)$ tem-se que $X$ e $F^{-1}(U)$ têm a mesma função distribuiçào.

Prova. De fato, uma vez que $F^{-1}(u)$ é não-decrescente segue que,

$$
P\left(F^{-1}(U) \leq x\right)=P(U \leq F(x))=F(x) .
$$


O tradicional método de simulação de Monte Carlo é baseado exatamente neste fato, que uma realização $x$ da variável aleatória $X$ com função distribuição $F$ pode ser obtida a partir de uma quantidade aleatória $u$ gerada de $U \sim U(0,1)$, e então fazer $x=F^{-1}(u)$.

\subsection{Cópulas}

É fato bem conhecido que a dependència entre as variáveis aleatórias $X_{1}, \ldots, X_{d}$ é completamente descrita pela função de distribuição conjunta $H$. Suponha que se transforme o vetor aleatório $\left(X_{1}, \ldots, X_{d}\right)$ componente a componente para que ele tenha distribuições marginais uniformes no intervalo $[0,1]$. Desse modo, o Teorema de Sklar, veja Sklar (1959), afirma que existe uma função $C$, denominada cópula $d$-dimensional que relaciona as funções distribuição marginais com a função distribuição conjunta.

Existem duas maneiras básicas de se caracterizar uma cópula. A primeira delas como uma função distribuição conjunta e outra como uma função que deve satisfazer a certas condições.

Definição 2.2. Uma cópula é uma função de distribuição conjunta

$$
C\left(u_{1}, \ldots, u_{d}\right)=P\left(U_{1} \leq u_{1}, \ldots, U_{d} \leq u_{d}\right),
$$

para todo $\left(u_{1}, \ldots, u_{d}\right) \in[0,1]^{d}$, cujas marginais são todas uniformes no intervalo $[0,1]$.

Portanto a condição que caracteriza uma função distribuição conjunta como cópula é que as marginais devem ser uniformes. Isto implica que toda cópula é uma função distribuição conjunta mas nem toda função distribuição conjunta é uma cópula. 
Definição 2.3. Uma função $C:[0,1]^{d} \mapsto[0,1]$ é uma cópula, se e somente se, ela satisfaz as seguintes condições:

(i) $C\left(u_{1}, \ldots, u_{i-1}, 0, u_{i+1}, \ldots, u_{d}\right)=0$, para todo $i \in\{1, \ldots, d\}$;

(ii) $C\left(1, \ldots, u_{i}, \ldots, 1\right)=u_{i}$, para todo $i \in\{1, \ldots, d\}$;

(iii) Para todo $\left(a_{1}, \ldots, a_{d}\right),\left(b_{1}, \ldots, b_{d}\right) \in[0,1]^{d}$ para o qual $a_{i} \leq b_{i}$ tem-se:

$$
\sum_{i_{1}=1}^{2} \cdots \sum_{i_{d}=1}^{2}(-1)^{i_{1}+\cdots+i_{d}} C\left(u_{1 i_{1}}, \ldots, u_{d i_{d}}\right) \geq 0
$$

com $u_{j 1}=a_{j}$ e $u_{j 2}=b_{j}, j \in\{1, \ldots, d\}$.

\section{Algumas propriedades das cópulas:}

(p1) Invariância sobre transformações estritamente crescentes, i.e., se $\alpha_{1}, \ldots, \alpha_{d}$ são funções estritamente crescentes, então

$$
C_{\alpha_{1}\left(X_{1}\right), \ldots, \alpha_{d}\left(X_{d}\right)}\left(u_{1}, \ldots, u_{d}\right)=C_{X_{1}, \ldots, \mathrm{X}_{d}}\left(u_{1}, \ldots, u_{d}\right)
$$

Este resultado também é válido para funções estritamente crescentes quase em toda parte;

(p2) Para todo $\left(u_{1}, \ldots, u_{d}\right) \in[0,1]^{d}$ tem-se:

$$
0 \leq \frac{\partial C\left(u_{1}, \ldots, u_{d}\right)}{\partial u_{i}} \leq 1
$$

para $i=1, \ldots, d$, veja Nelsen $(1999)$;

(p3) Para toda cópula $d$-dimensional $C$ e para todo $\left(u_{1}, \ldots, u_{d}\right) \in\left[0,1^{d}{ }^{d}\right.$ tem-se:

$$
\max \left(\sum_{i=1}^{d} u_{i}-d+1,0\right) \leq C\left(u_{1}, \ldots, u_{d}\right) \leq \min \left(u_{1}, \ldots, u_{d}\right) .
$$

Estes limites são chamados de limites inferior e superior de Fréchet e denotados por $W\left(u_{1}, \ldots, u_{d}\right)$ e $M\left(u_{1}, \ldots, u_{d}\right)$ respectivamente. 
Observação 2.3. Não é difícil verificar que $M\left(u_{1}, \ldots, u_{d}\right)$ é uma cópula para todo $d \geq 2$, enquanto que $W\left(u_{1}, \ldots, u_{d}\right)$ é uma cópula apenas para $d=2$.

Observação 2.4. Pode-se verificar também, veja Nelsen (1999, Seção 2.5), que se $U$ e $V$ são v.a.'s uniformes em $[0,1]$ então:

- Se a função distribuição conjunta de $(U, V)$ é $M(u, v)$ então $P(U=V)=1$, neste caso o vetor $(U, V)$ é chamado comonotônico;

- Se a função distribuição conjunta de $(U, V)$ é $W(u, v)$ então $P(U=1-V)=1$, e neste cas̀o o vetor $(U, V)$ é chamado contra-comonotônico.

Teorema 2.1 (Teorema de Sklar). Seja H uma função distribuição conjunta com marginais $F_{X_{1}}, \ldots, F_{X_{d}}$. Então existe uma cópula d-dimensional $C$ tal que,

$$
H\left(x_{1}, \ldots, x_{d}\right)=C\left(F_{X_{1}}\left(x_{1}\right), \ldots, F_{X_{d}}\left(x_{d}\right)\right)
$$

para todo $\left(x_{1}, \ldots, x_{d}\right) \in[-\infty, \infty]^{d}$. Se $F_{X_{1}}, \ldots, F_{X_{d}}$ forem contínuas, então $C$ é única, caso contrário $C$ é unicamente determinada no conjunto $\operatorname{Im}\left(F_{X_{1}}\right) \times \ldots \times$ $\operatorname{Im}\left(F_{X_{d}}\right)$, onde $\operatorname{Im}\left(F_{X_{i}}\right)$ representa o conjunto imagem da função $F_{X_{i}}$. Portanto a cópula d-dimensional $C$ associada a $H$ é dada por

$$
C\left(u_{1}, \ldots, u_{d}\right)=H\left(F_{X_{1}}^{-1}\left(u_{1}\right), \ldots, F_{X_{d}}^{-1}\left(u_{d}\right)\right)
$$

para todo $\left(u_{1}, \ldots, u_{d}\right) \in[0,1]^{d}$, em que $F_{X_{i}}^{-1}$ é a inversa de $F_{X_{i}}$ como definido em (2.1).

A recíproca do Teorema de Sklar é a seguinte: Seja $C$ uma função satisfazendo as condições da Definição 2.3 e $F_{X_{1}}, \ldots, F_{X_{d}}$ funções distribuição, então a função $H$ definida pela relação (2.2) é uma função distribuição conjunta com marginais $F_{X_{1}}, \ldots, F_{X_{d}}$, veja Nelsen (1999). 
A recíproca do Teorema de Sklar implica que podemos acoplar distribuições univariadas, de qualquer tipo, com qualquer cópula, e teremos definido uma distribuição multivariada válida. Este resultado é útil porque usualmente temos alguma idéia sobre as distribuições univariadas, mas nossa intuição raramente indica a escolha de distribuições multivariadas disponíveis. Outro fato importante é que podemos estudar o fenòmeno de dependência entre as variáveis sem fixar as distribuições marginais.

A classe de cópulas Arquimedianas provavelmente seja a mais utilizada na prática. Essa classe de cópulas surgiu inicialmente no estudo de espaços métricos probabilísticos como $t$-normas ( $t$-norma vem de norma triangular) que satisfaziam a propriedade $T(u, u)<u$ para todo $u \in(0,1)$. No entanto cabe ressaltar que nem toda $t$-norma é uma cópula e nem toda cópula é uma $t$-norma. Para mais detalhes veja Schweizer e Sklar (1983).

A classe de cópulas Arquimedianas pode ser definida da seguinte forma:

$$
C\left(u_{1}, \ldots, u_{d}\right)= \begin{cases}\varphi^{-1}\left(\varphi\left(u_{1}\right)+\cdots+\varphi\left(u_{d}\right)\right) & \text { se } \sum_{i=1}^{d} \varphi\left(u_{i}\right) \leq \varphi(0) \\ 0 & \text { caso contrário, }\end{cases}
$$

onde a função $\varphi$ é chamada de geradora da respectiva família e deve satisfazer as seguintes condições: $\varphi(1)=0$ e $\varphi(t)$ é decrescente e convexa para todo $t \in(0,1)$, i.e., $\varphi^{\prime}(t)<0$ e $\varphi^{\prime \prime}(t) \geq 0$.

Esta classe de cópulas foi exaustivamente estudada em Genest e Mackay (1986) e Genest e Rivest (1993). Como foi demonstrado nestes artigos, a família de cópulas Arquimedianas possui propriedades interessantes que as tornam atraentes para a inferència estatística e para a simulação.

As seguintes propriedades para ıma cópula Arquimediana bivariada $C$, também são válidas para o caso multivariado: 
(p1) $C$ é simétrica (permutável), isto é $C(u, v)=C(v, u)$ para qualquer $(u, v) \in$ $[0,1]^{2}$

(p2) $C$ é associativa, isto é, $C(C(u, v), w)=C(u, C(v, w))$ para todo $u, v, w \mathrm{em}$ $[0,1]$

(p3) Se $\varphi$ é a geradora de $C$, então para alguma constante $k>0$ tem-se que $k \varphi$ é também uma geradora de $C$.

\subsection{Transformada de nível}

O Teorema de Sklar só garante a unicidade da cópula se as funções distribuição marginais forem contínuas. Recentemente em Kowalczyk (2000) e Niewiadomska-Bugaj e Kowalczyk (2005), a transformada de nível é utilizada para se obter a unicidade mesmo no caso em que não temos funções distribuição marginais contínuas. A transformada de nível pode ser interpretada como uma extensão da transformada integral da probabilidade, e por isso utilizaremos a mesma notação $\mathcal{I}(F)(u)$.

Definição 2.4. A transformada de nível da função distribuição $F$ da variável aleatória $X$ é dada por

$$
\mathcal{I}(F)(u)=\int_{-\infty}^{+\infty} I^{*}(F(x), u) d F(x)=E\left[I^{*}(F(X), u)\right], \text { para } u \in[0,1],
$$

em que

$$
I^{*}(F(x), u)= \begin{cases}0 & \text { se } F(x-)>u \\ \frac{u-F(x-)}{F(x)-F(x-)} & \text { se } F(x-) \leq u<F(x) \\ 1 & \text { se } F(x) \leq u\end{cases}
$$

Note que se $F$ for contínua então $F(x-)=F(x)$ e portanto

$$
I^{*}(F(x), u)=I(F(x), u)= \begin{cases}0 & \text { se } F(x)>u \\ 1 & \text { se } F(x) \leq u\end{cases}
$$


Exemplo 2.2 (continuação do exemplo 2.1). A função distribuição $F$ da variável aleatória $X$ contém dois pontos de descontinuidade, um em $X=0$ e outro em $X=1$. Assim,

$$
I^{*}(F(0), u)=\left\{\begin{array}{ll}
0 & \text { se } 0>u \\
\frac{u}{p} & \text { se } 0 \leq u<p \\
1 & \text { se } p \leq u
\end{array} \quad \text { e } \quad I^{*}(F(1), u)= \begin{cases}0 & \text { se } p>u \\
\frac{u-p}{1-p} & \text { se } p \leq u<1 \\
1 & \text { se } 1 \leq u\end{cases}\right.
$$

Deste modo,

$$
\begin{aligned}
\mathcal{I}(F)(u) & =\int_{-\infty}^{+\infty} I^{*}(F(x), u) d F(x) \\
& =p I^{*}(F(0), u)+(1-p) I^{*}(F(1), u) \\
& =p I^{*}(p, u)+(1-p) I^{*}(1, u) .
\end{aligned}
$$

Portanto,

$$
\mathcal{I}(F)(u)= \begin{cases}\frac{p(u-0)}{p-0}=u & \text { para } u \in[0, p) \\ p+(1-p) \frac{u-p}{1-p}=u & \text { para } u \in[p, 1) \\ 1 & \text { para } u=1\end{cases}
$$

Nestas condições, $\mathcal{I}(F)(u)=P(U \leq u)=u$ para todo $u \in[0,1]$.

Fazendo uso da transformada de nível, a relação (2.3) pode ser reescrita como

$$
C\left(u_{1}, \ldots, u_{d}\right)=\int_{[-\infty, \infty]^{d}} \prod_{i=1}^{d} I_{i}^{*}\left(F_{X_{i}}\left(x_{i}\right), u_{i}\right) d H\left(x_{1}, \ldots, x_{d}\right),
$$

em que

$$
I_{i}^{*}\left(F_{X_{i}}\left(x_{i}\right), u_{i}\right)= \begin{cases}0 & \text { se } F_{Y_{i}}\left(x_{i}-\right)>u_{i} \\ \frac{u_{i}-F_{X_{i}}\left(x_{i}-\right)}{F_{X_{i}}\left(x_{i}\right)-F_{X_{i}}\left(x_{i}-\right)} & \text { se } F_{X_{i}}\left(x_{i}-\right) \leq u_{i}<F_{Y_{i}}\left(x_{i}\right) \\ 1 & \text { se } F_{X_{i}}\left(x_{i}\right) \leq u_{i}\end{cases}
$$

para todo $i=1, \ldots, d$. 


\subsection{O fenômeno da dependência}

Quando estudamos o fenòmeno da dependência é importante tratarmos de dois casos extremos: comonotonicidade e contra-monotonicidade e sobre vetores concordantes e discordante. Faremos também uma exposição sobre as medidas de dependência global: coeficientes de Kendall, Spearman e Blest.

\subsubsection{Comonotonicidade e contra-monotonicidade}

Definição 2.5 (Comonotonicidade - caso bivariado). As variáveis aleatórias $X$ e Y são ditas comonotônicas se são positivamente perfeitamente dependentes, i.e., $P(X=Y)=1$ e contra-monotônicas se são negativamente perfeitamente dependentes, i.e., $P(X=-Y)=1$.

A dependència perfeita está fortemente relacionada com a noção de comonotonicidade e contra-monotonicidade, introduzidas na Definição 2.5 no caso bivariado.

Nas observações 2.3 e 2.4, ressaltamos que os limites inferior $W(u, v)=\max (u+$ $v-1,0)$ e superior $M(u, v)=\min (u, v)$ de Fréchet são cópulas e configuram as funções distribuições conjuntas bivariadas dos vetores aleatórios $(U, 1-U)$ e $(U, U)$ respectivamente, em que $U \sim U(0,1)$. Neste caso $W$ e $M$ descrevem as dependencias perfeitas negativa e positiva.

Teorema 2.2 (Embrechts et al. (2002)). Seja $(X, Y)$ com cópula $W$ ou $M$. Então existem duas funções monótonas $\alpha, \beta:[-\infty, \infty] \mapsto[-\infty, \infty]$ e uma variável aleatória $Z$ tal que,

$$
(X, Y) \stackrel{i}{=}(\alpha(Z), \beta(Z))
$$

onde $\stackrel{d}{=}$ indica igualdade em distribuição e com $\alpha$ crescente e $\beta$ decrescente no caso 
de $(X, Y)$ ter cópula $W$ e com $\alpha$ e $\beta$ crescentes no caso de $(X, Y)$ ter cópula $M$. A volta do teorema também é válida.

Para estender a noção de comonotonicidade dada na Definição 2.5 para $n \geq 2$, precisaremos da seguinte definição de conjunto comonotônico.

Definição 2.6 (conjunto comonotônico). Um conjunto em $[-\infty, \infty]^{d}$ é comonotônico se para todos $\left(x_{1}, \ldots, x_{d}\right) e\left(y_{1}, \ldots, y_{d}\right)$ neste conjunto, $x_{i}<y_{i}$ implica $x_{j} \leq y_{j}$ para todo $j \in\{1, \ldots, d\}$. Quando a imagem de um vetor aleatório é um. conjunto comonotônico, também o vetor aleatório e sua distribuição são chamados comonotônicos.

Pode-se concluir da definição acima que a comonotonicidade é uma estrutura de dependência positiva muito forte. A comonotonicidade pode ser interpretada como a extensão do conceito de correlação positiva perfeita.

No seguinte teorema várias caracterizações equivalentes para comonotonicidade são dadas.

Teorema 2.3 (Dhaene et al. (2002)). O vetor aleatório $\left(X_{1}, \ldots, X_{d}\right)$ é comonotônico, se e somente se, uma das seguintes condições equivalentes são satisfeitas:

(c1) O vetor aleatório $\left(X_{1}, \ldots, X_{d}\right)$ é comonotônico;

(c2) Para todos $\left(x_{1}, \ldots, x_{d}\right) \in[-\infty, \infty]^{d}$ temos

$$
H\left(x_{1}, \ldots, x_{d}\right)=\min \left\{F_{X_{1}}\left(x_{1}\right), \ldots, F_{X_{d}}\left(x_{d}\right)\right\} ;
$$

(c3) $\left(X_{1}, \ldots, X_{d}\right) \stackrel{d}{=}\left(F_{X_{1}}^{-1}(U), \ldots, F_{X_{d}}^{-1}(U)\right)$ para $U \sim U(0,1)$; 
(c4) Existem funções não decrescentes $\alpha_{i}:[-\infty, \infty] \mapsto[-\infty, \infty], i=1, \ldots, d$ e uma variável aleatória $Z$ tal que,

$$
\left(X_{1}, \ldots, X_{d}\right) \stackrel{d}{=}\left(\alpha_{1}(Z), \ldots, \alpha_{d}(Z)\right)
$$

Portanto, o vetor aleatório $\mathbf{X}=\left(X_{1}, \ldots, X_{n}\right)$ é comonotônico se e somente se sua distribuição conjunta coincide com o limite superior de Fréchet.

Note que de maneira similar pode-se definir um conjunto contra-monotônico e então fazer sua correspondente caracterização.

\subsubsection{Medidas de dependência}

Embora a dependência entre variáveis aleatórias seja completamente descrita pela sua função distribuição conjunta $H$, a distribuição conjunta não informa especificamente como é essa dependência, nem a mensura, i.e., a distribuição conjunta não mostra explicitamente se as variáveis são por exemplo positivamente ou negativamente relacionadas. Para podermos compreender melhor estas características e outras mais, é que foram desenvolvidas as medidas de dependência, que são instrumentos que quantificam essas relações. Essa quantificação tem como base um intervalo entre -1 e +1 , valores que representam respectivamente a dependência perfeita negativa e a dependencia perfeita positiva e entre estes dois extremos a associação entre as variáveis aleatórias tem uma infinidade de outras formas, por exemplo dependència linear, quadrática, simétrica, caudal, etc.

As medidas de dependència entretanto tèm uma natureza bastante específica e resumida, i.e., cada medida se destina a captar determinados aspectos da estru- 
tura dependência em detrimento de outros que as variáveis aleatórias em particular possam apresentar, como por exemplo: o coeficiente de correlação linear que mede unicamente o grau de correlação linear entre as variáveis, o coeficiente de Kendall e Spearman que medem o grau de concordância entre as variáveis. Além disso, uma medida de dependência fornece unicamente uma média global. Portanto, muito cuidado deve ser tomado na utilização de medidas de dependência desta natureza, pois as mesmas podem levar a conclusões e interpretações errôneas. No Capítulo 3 vamos voltar a falar sobre estes problemas das medidas globais e mostrar alternativas a estas medidas que são as medidas de dependência locais. A seguir apresentamos algumas propriedades das medidas globais de dependência.

\section{Propriedades das medidas de dependência:}

Toda medida de dependência, possui um conjunto de propriedades desejáveis. Seja $\delta$ uma medida de dependência, então:

(p1) $\delta$ é simétrica se $\delta(X, Y)=\delta(Y, X)$;

(p2) $\delta$ é "normalizada"se $-1 \leq \delta(X, Y) \leq 1$;

(p3) $\delta(X, Y)=1 \Leftrightarrow(X, Y)$ é comonotônico e $\delta(X, Y)=-1 \Leftrightarrow(X, Y)$ é contramonotònico;

(p4) $\delta(T(X), Y)=\delta(X, Y)$, para $T$ crescente, $\delta(T(X), Y)=-\delta(X, Y)$, para $T$ decrescente;

(p5) $\delta(X, Y)=0 \Leftrightarrow(X, Y)$ são independentes. 


\subsubsection{Concordância e Medidas de Concordância}

Sejam $(x, y)$ e $\left(x^{\prime}, y^{\prime}\right)$ duas observações do vetor $(X, Y)$ de variáveis aleatórias contínuas. Então $(x, y)$ e $\left(x^{\prime}, y^{\prime}\right)$ são concordantes quando $\left(x-x^{\prime}\right)\left(y-y^{\prime}\right)>0$ e discordantes quando $\left(x-x^{\prime}\right)\left(y-y^{\prime}\right)<0$.

Teorema 2.4 (Nelsen $\left(1999\right.$, p. 127)). Sejam $\left(X_{1}, Y_{1}\right)$ e $\left(X_{2}, Y_{2}\right)$ vetores independentes de variáveis aleatórias contínuas, com funções distribuição conjuntas $H_{1}$ e $H_{2}$, respectivamente, com marginais comuns $F$ (de $X_{1}$ e $X_{2}$ ) e $G$ (de $Y_{1}$ e $\left.Y_{2}\right)$. Sejam $C_{1}$ e $C_{2}$ as cópulas de $\left(X_{1}, Y_{1}\right)$ e $\left(X_{2}, Y_{2}\right)$ respectivamente de modo que $H_{1}\left(x_{1}, y_{1}\right)=C_{1}\left(F\left(x_{1}\right), G\left(y_{1}\right)\right)$ e $H_{2}\left(x_{2}, y_{2}\right)=C_{2}\left(F\left(x_{2}\right), G\left(y_{2}\right)\right)$. Seja $Q$ a diferença entre as probabilidades de concordância e discordância entre $\left(X_{1}, Y_{1}\right)$ e $\left(X_{2}, Y_{2}\right)$, isto é, seja,

$$
Q=P\left[\left(X_{1}-X_{2}\right)\left(Y_{1}-Y_{2}\right)>0\right]-P\left[\left(X_{1}-X_{2}\right)\left(Y_{1}-Y_{2}\right)<0\right]
$$

então,

$$
Q=Q\left(C_{1}, C_{2}\right)=4 \int_{0}^{1} \int_{0}^{1} C_{2}(u, v) d C_{1}(u, v)-1
$$

Corolário 2.1 (Nelsen $\left(1999\right.$, p. 128)). Sejam $C_{1}, C_{2}$ e $Q$ como definidos no Teorema 2.4 , então,

(i) $Q$ é simétrica em seus argumentos se $Q\left(C_{1}, C_{2}\right)=Q\left(C_{2}, C_{1}\right)$;

(ii) $Q$ é não decrescente em cada argumento, i.e., se $C_{1} \prec C_{1}^{\prime}$ e $C_{2} \prec C_{2}^{\prime}$, então $Q\left(C_{1}, C_{2}\right) \leq Q\left(C_{1}^{\prime}, C_{2}^{\prime}\right)$. Em que, $C_{i}^{\prime} \prec C_{i}$ se $C_{i}^{\prime}(u, v) \leq C_{i}(u, v)$ para todo $(u, v) \in[0,1]^{2}$. 
Definição 2.7 (Nelsen (1999, p. 136)). Uma medida de dependência $\kappa$ entre duas variáveis aleatórias contínuas $X$ e $Y$ com cópula associada $C$ é uma medida de concordancia se ela satisfaz as seguintes propriedades:

(p1) k está definida para todo par $(X, Y)$ de variáveis aleatórias contínuas;

(p2) $-1 \leq \kappa_{X, Y} \leq 1, \kappa_{X, X}=1$ e $\kappa_{X,-X}=-1$;

(p3) $\kappa_{X, Y}=\kappa_{Y, X}$;

(p4) Se $X$ e $Y$ são independentes, então $\kappa_{X, Y}=\kappa_{\Pi}=0$, em que $\Pi(u, v)=u v$ é a cópula da dependência também chamada de cópula produto;

(p5) $\kappa_{-X, Y}=\kappa_{X,-Y}=-\kappa_{X, Y}$;

(p6) $S e C$ e $C^{\prime}$ são cópulas tais que $C \prec C^{\prime}$, então $\kappa_{C} \leq \kappa_{C^{\prime}}$;

(p7) Se $\left\{\left(X_{n}, Y_{n}\right)\right\}$ é uma seqüência de variáveis aleatórias contínuas com cópulas $C_{n}$, e se $\left\{C_{n}\right\}$ converge ponto a ponto para $C$, então $\lim _{n \rightarrow \infty} \kappa_{C_{n}}=\kappa_{C}$.

Como conseqüència da Definição 2.7, se $Y$ é quase certamente uma função crescente de $X$, então $\kappa_{X, Y}=\kappa_{M}=1$, e se $Y$ é quase certamente uma função decrescente de $X$. então $\kappa_{X, Y}=\kappa_{W}=-1$. Além disso, se $\alpha$ e $\beta$ são quase certamente funções crescentes na $\operatorname{Im}(X)$ e $\operatorname{Im}(Y)$ respectivamente, então $\kappa_{\alpha(X), \beta(Y)}=\kappa_{X, Y}$.

Definição 2.8 (Coeficiente de Kendall). Seja $(X, Y)$ um vetor aleatório com cópula associada $C$. Então o coeficiente de Kendall $\tau(X, Y)$ do vetor aleatório $(X, Y)$ em termos da cópula correspondente $C$ é dado por

$$
\tau(X, Y)=4 \int_{0}^{1} \int_{0}^{1} C(u, v) d C(u, v)-1 .
$$


A versão amostral $\tau_{n}$ de $\tau(X, Y)$ para uma amostra aleatória $\left\{\left(X_{1}, Y_{1}\right), \ldots,\left(X_{n}, Y_{n}\right)\right\}$ é dada por

$$
\tau_{n}=\frac{c-d}{\left(\begin{array}{c}
n \\
2
\end{array}\right)}
$$

em que $c$ é o número de pares concordantes, i.e., o número de pares para o qual $\left\{X_{i} \leq X_{j}, Y_{i} \leq Y_{j}\right\}$ ou $\left\{X_{i}>X_{j}, Y_{i}>Y_{j}\right\}$ e $d$ é o número de pares discordantes, i.e., o número de pares para o qual $\left\{X_{i} \leq X_{j}, Y_{i}>Y_{j}\right\}$ ou $\left\{X_{i}>X_{j}, Y_{i} \leq Y_{j}\right\}$.

Definição 2.9 (Coeficiente de Spearman). Seja $(X, Y)$ um vetor aleatório com cópula associada $C$. Então o coeficiente de Spearman $\rho(X, Y)$ do vetor aleatório $(X, Y)$ em termos da cópula correspondente $C$ é dado por

$$
\rho(X, Y)=12 \int_{0}^{1} \int_{0}^{1} C(u, v) d u d v-3
$$

A versão amostral $\rho_{n}$ de $\rho(X, Y)$ e dada por

$$
\rho_{n}=\frac{12}{n^{3}-n} \sum_{i=1}^{n} R_{X_{i}} R_{Y_{i}}-\frac{3(n+1)}{n-1} .
$$

em que $R_{X_{i}}$ e $R_{Y_{i}}$ são os postos de $X_{i}$ e $Y_{i}$ respectivamente.

As medidas de dependência não-paramétricas clássicas de Kendall e de Spearman são melhores que o coeficiente de correlação de Pearson $r(X, Y)=\operatorname{Corr}(X, Y)$ no caso geral quando não temos uma correlação linear entre as variáveis, pois estas medidas sempre existem e assumem seus valores extremos \pm 1 quando $X$ e $Y$ estão em perfeita dependência funcional monótona. Assim, quando consideramos uma amostra aleatória $\left\{\left(X_{1}, Y_{1}\right), \ldots,\left(X_{n}, Y_{n}\right)\right\}$ provenientes de uma distribuição desconhecida $H$ cujo suporte é $[0, \infty)^{2}$, os valores dos coeficientes de $\rho(X, Y)$ e de $\tau(X, Y)$ não tèm restrições, ao passo que o coeficiente de correlação $r(X, Y)$ só pode 
cobrir um intervalo $\left[r_{\min }, r_{\max }\right] \in[-1,1]$ cujos limites dependem da escolha das marginais $F$ e $G$. Para um discussão mais detalhada sobre as vantagens das medidas de dependência baseadas em postos ver Embrechts et al. (2002).

Notando que os coeficientes de Kendall e Spearman amostrais, $\rho_{n}$ e $\tau_{n}$, colocam o mesmo peso nas diferenças dos postos $R_{X_{i}}-R_{Y_{i}}, \quad 1 \leq i \leq n$, Blest (2000) propôs uma medida alternativa de correlação de postos não paramétrica, em que as diferenças nos postos extremos recebem um peso maior, pois pareceriam ser mais críticos, e portanto essas discrepâncias deveriam ser ressaltadas.

Definição 2.10 (Blest (2000)). Seja $(X, Y)$ um vetor aleatório com cópula $C$ associada. Então o coeficiente de Blest, $\nu(X, Y)$, do vetor aleatório $(X, Y) \mathrm{em}$ termos da cópula correspondente $C$ é dado por

$$
\nu(X, Y)=2-12\left(\int_{0}^{1} \int_{0}^{1}(1-u)^{2} v d C(u, v)\right),
$$

A versão amostral do coeficiente de Blest $\nu_{n}$, é dado por,

$$
\nu_{n}=\frac{2 n+1}{n-1}-\frac{12}{n^{2}-n} \sum_{i=1}^{n} R_{Y_{i}}\left(1-\frac{R_{X_{i}}}{n+1}\right)^{2} \text {. }
$$

O coeficiente $\nu_{n}$ é mais extremo quando os postos coincidem $\left(R_{X_{i}}=R_{Y_{i}}\right)$ ou quando $\left(R_{X_{i}}=n+1-R_{Y_{i}}\right)$. Blest (2000) mostra que $\nu_{n}$ pode discriminar mais facilmente do que os coeficientes $\rho_{n}$ ou $\tau_{n}$, ainda que seja altamente correlacionado com ambos.

A medida $\nu(X, Y)$ não é uma medida de concordância, uma vez que em geral $\nu(X, Y) \neq \nu(Y, X)$. Note entretanto que $\nu(X,-Y)=-\nu(X, Y)$ (por envolver a cópula $u-C(u, 1-v)$ do par $(X,-Y))$, veja Genest e Plante (2003). 


\section{Capítulo 3}

\section{Representação para cópulas bivariadas via medida de dependência local}

Nos últimos anos tem-se observado um grande avanço na teoria de cópulas motivados pelas várias aplicações em probabilidade, estatística, finanças e atuária. Entretanto, a escolha de uma estrutura de dependência, representada pela cópula, não é uma tarefa fácil. Um dos motivos é que uma cópula não nos informa de maneira explícita como é a forma da estrutura de dependência.

O objetivo deste capítulo é formular uma nova representação em que a função que representa a dependência mostre explicitamente a estrutura de dependência escolhida.

A idéia básica é representar uma cópula utilizando uma medida de dependência local. Em geral, as medidas de dependência locais são atrativas porque elas fornecem ıma radiografia detalhada da estrutura local de dependência, o que não ocorre com as medidas globais. As medidas de dependência locais são usualmente definidas como uma correlação entre $X$ e $Y$ dado $X=x$ e $Y=y$ (ou dado $X \leq x$ e 
$Y \leq y$ ), veja Bjerve e Doksum (1993), Doksum et al. (1994). Por exemplo, Kotz e Nadarajah (2003) propuseram uma nova medida local derivada do coeficiente de correlação linear como segue,

$$
\gamma(x, y)=\frac{E([X-E(X \mid Y=y)][Y-E(Y \mid X=x)])}{\sqrt{E[X-E(X \mid Y=y)]^{2} E[Y-E(Y \mid X=x)]^{2}}}, \quad(x, y) \in[-\infty, \infty]^{2} .
$$

A medida $\gamma(x, y)$ é uma generalização radical do usual coeficiente de correlação linear de Pearson e caracteriza o efeito de $X$ sobre $Y$ dado $Y=y$ e o efeito de $Y$ sobre $X$ dado $X=x$. Kotz e Nadarajah (2003) mostram que este coeficiente de correlação "condicional" contorna algumas fragilidades que outras medidas locais de dependência conhecidas possuem, como por exemplo, serem constantes para certas famílias de distribuição veja Jones $(1996,1998)$. Em Arnold et al. (1999) são expostos outros problemas das medidas de dependência locais.

Entretanto, a medida de dependência local $\gamma(x, y)$ é influenciada pela distribuições marginais de $X$ e $Y$. Isto significa que para uma mesma estrutura de dependência uma mudança nas distribuições marginais poderá implicar numa mudança no valor da medida. Todavia, pelo Teorema de Sklar sabemos que uma estrutura de dependência não é influenciada pelas marginais. Portanto, a medida $\gamma(x, y)$ não é adequada sob o ponto de vista do Teorema de Sklar para descrever uma estrutura de dependência.

Com o objetivo de contornar este problema, consideremos uma simples modificação, baseado no fato que o coeficiente de Spearman $\rho_{S}$ de $(X, Y)$ é igual ao coeficiente de correlação de Pearson entre $F(X)$ e $G(Y)$, i.e.

$$
\rho_{S}=\frac{E([U-E(U)][V-E(V)])}{\sqrt{\operatorname{Var}(U) \operatorname{Var}(V)}}=12 \int_{0}^{1} \int_{0}^{1}[C(u, v)-u v] d u d v,
$$


veja Nelsen (1999, p. 138). Portanto, uma versão alternativa de $\gamma(x, y)$ pode ser dado por

$$
\gamma_{S}(u, v)=\frac{E([U-E(U \mid V=v)][V-E(V \mid U=u)])}{\sqrt{E[U-E(U \mid V=v)]^{2} E[V-E(V \mid U=u)]^{2}}}, \quad(u, v) \in[0,1]^{2},
$$

A medida $\gamma_{S}(u, v)$ pode ser interpretada como um coeficiente de Spearman condicional. Entretanto, apesar desta medida ser interessante, não conseguimos uma representação explícita para cópulas baseada nesta medida, isto é, não conseguimos de um modo simples caracterizar uma cópula utilizando esta medida.

Em face do exposto, na Seção 3.1 apresentamos uma medida de dependência local que tem um representação explícita para cópulas. A importância desta representação é que podemos decompor qualquer cópula em duas partes: as marginais uniformes e a estrutura de dependência resumida pela medida de dependência local que pode ser interpretada com um coeficiente de Spearman "local" (mas nào condicional como $\gamma_{S}$ ). Chamamos esta medida de função Spearman e denotamos por $\rho_{C}$. Demonstramos que para toda cópula $C$ sempre existe uma função Spearman $\rho_{C}$ associada. Deste modo, a partir deste resultado podemos caracterizar uma cópula pela caracterização da sua função Spearman associada e vice-versa. A vantagem é que $\rho_{C}$ fornece uma informação precisa e explícita da estrutura de dependencia, o que não ocorre com a cópula. Com isto pode-se construir critérios baseados nesta nova medida que poderão auxiliar na difícil tarefa de escolha da função que melhor representa a estrutura de dependência correspondente.

Na Seção 3.2, exibimos algumas propriedades da função Spearman $\rho_{C}$ e na Seçào 3.3. apresentamos uma outra medida de dependencia local baseada no coeficiente de correlação linear que pode ser utilizada em conjunto com a cópula gaussiana e exibimos algumas propriedades. 


\subsection{Uma nova representação para cópulas}

A seguir apresentamos a definição da medida de dependència local que denominaremos Função Spearman e denotamos $\rho_{C}$.

Definição 3.1 (Função Spearman). Seja $(U, V)$ um vetor aleatório bivariado com marginais uniformes $U \sim U(0,1)$ e $V \sim U(0,1)$ e função distribuição conjunta $P(U \leq u, V \leq v)=C(u, v)$. Seja,

$$
Z_{u}=\mathbf{I}\{U \leq u\} \quad \text { e } \quad Z_{v}=\mathbf{I}\{V \leq v\}
$$

definidas para todo par $(u, v) \in[0,1]^{2}$, em que $\mathbf{I}\{A\}$ é a função indicadora do conjunto A. Então, a função Spearman é o coeficiente de correlação entre $Z_{u}$ e $Z_{v}$ que é dado por,

$$
\rho_{C}(u, v)=\rho_{Z_{u}, Z_{v}}=\frac{C(u, v)-u v}{\sqrt{u v(1-u)(1-v)}}
$$

A seguinte proposição é válida para toda cópula bivariada $C$ e toda função distribuição conjunta $H$ com marginais $F$ e $G$.

Proposição 3.1. Seja $C$ uma cópula. Então existe uma única função $\rho_{C}:[0,1]^{2} \mapsto$ $[-1,1]$ contínua, tal que para todo $(u, v) \in[0,1]^{2}$, tem-se

$$
C(u, v)=u v+\rho_{C}(u, v) \sqrt{u v(1-u)(1-v)} .
$$

Equivalentemente tem-se que, dada uma função distribuição conjunta $H$ com marginais $F$ e $G$, existe uma função $\rho_{H}:[-\infty, \infty]^{2} \mapsto[-1,1]$ contínua, tal que para todo $(x, y) \in[-\infty, \infty]^{2}$,

$$
H(x, y)=F(x) G(y)+\rho_{H}(F(x), G(y)) \sqrt{F(x) G(y)(1-F(x))(1-G(y))} .
$$


Se as marginais $F$ e $G$ forem contínuas então $\rho_{H}$ é única, caso contrário será unicamente determinada sobre o conjunto imagem $\operatorname{Im} F \times \operatorname{ImG}$. Além do mais, se $H$ tem cópula associada $C$ então

$$
\rho_{H}(F(x), G(y))=\rho_{C}(u, v)
$$

para todo $(x, y) \in[-\infty, \infty]^{2}$ tal que $u=F(x)$ e $v=G(y)$

Prova. A prova desta proposição decorre diretamente da Definição 3.1. Deste. modo, da relação (3.1) obtemos a representação para cópulas em termos da função Spearman $\rho_{C}$,

$$
C(u, v)=u v+\rho_{C}(u, v) \sqrt{u v(1-u)(1-v)}
$$

Note que, a função Spearman $\rho_{C}$ é função somente do par $(u, v)$ e da cópula associada $C(u, v)$. Assim, como $C$ é contínua, segue da relação (3.1) que $\rho_{C}$ também é contínua.

A relação (3.3) para funções distribuição bivariadas $H$ é conseqüência direta do Teorema de Sklar.

Para provar a unicidade de $\rho_{C}$, suponha que $\rho_{C}$ e $\rho_{C}^{\prime}$ sejam duas funções Spearman associadas a mesma cópula $C$. Então de (3.2) tem-se que isto só é possivel se e somente se $\rho_{C}(u, v)=\rho_{C}^{\prime}(u, v)$ para todo $(u, v) \in[0,1]^{2}$.

Observação 3.1. Pela expressão (3.1) pode-se concluir que $\rho_{C}$ é uma medida de dependência local que fornece para cada par $(u, v) \in[0,1]^{2}$ uma medida do quão afastada uma cópula $C$ está da cópula da independência $\Pi(u, v)=u v$.

Observação 3.2. A medida de dependência local $\rho_{C}$ pode interpretado como um Spearman "local". Para podermos mostrar a conexão entre a função Spearman $\rho_{C} e$ 
o coeficiente de Spearman $\rho_{S}$, primeiro note que se substituirmos $g(u, v)=C(u, v)-$ $u v$, então,

$$
\rho_{S}=12 \int_{0}^{1} \int_{0}^{1} g(u, v) d u d v
$$

veja Nelsen (1999, p. 138). Portanto, $g(u, v)$ poderia ser um candidato para a medida de dependência local, entretanto, sua imagem não é o intervalo $[-1,1]$. O fator $\sqrt{u v(1-u)(1-v)}$ "normaliza" a função $g(u, v)$, i.e., $\frac{g(u, v)}{\sqrt{u v(1-u)(1-v)}}$ é uma função limitada no intervalo $[-1,1]$. Esta "normalização" é importante para que possamos comparar $\rho_{C}$ com outras medidas de dependência que também são normalizadas.

Pode ser verificado facilmente que $\rho_{S}$ e $\rho_{C}$ têm a seguinte relação,

$$
\rho_{S}=12 \int_{0}^{1} \int_{0}^{1} \rho_{C}(u, v) \sqrt{u v(1-u)(1-v)} d u d v
$$

A função Spearman $\rho_{C}$ também pode ser vista como uma versão da medida de dependência local de quadrante, veja Nelsen (1999, p. 153).

A função Spearman $\rho_{C}$ também poderia ser chamada de função "SchweizerWolff", ou função "Blomqvist" pois $\rho_{C}(1 / 2,1 / 2)=4 C(1 / 2,1 / 2)-1=\beta$, ou função "Hoeffding" (veja (5.3.1) na pg. 171, (5.1.27) na pg. 148 e (5.3.4) na pg. 172, respectivamente em Nelsen (1999)), ou qualquer outro coeficiente baseado na quantidade $C(u, v)-u v$.

O próximo resultado é uma conseqüência direta da Proposição 3.1.

Corolário 3.1. Sejam $C_{1}$ e $C_{2}$ duas cópulas. Se $\rho_{C_{1}}(u, v)=\rho_{C_{2}}(u, v)$ para todo $(u, v) \in[0,1]^{2}$, então $C_{1}(u, v)=C_{2}(u, v)$ para todo $(u, v) \in[0,1]^{2}$.

A seguir damos dois exemplos para melhor compreensão da função Spearman $\rho_{C}$. 
Exemplo 3.1. Consideremos a cópula da família de Farlie-Gumbel-Morgenstein, i.e., $C(u, v)=u v+\theta u v(1-u)(1-v),-1 \leq \theta \leq 1$, veja Nelsen (1999, p. 68). Da relação (3.1) obtemos a seguinte função Spearman associada

$$
\rho_{C}(u, v)=\theta \sqrt{u v(1-u)(1-v)}
$$

para todo $(u, v) \in[0,1]^{2}$. Neste caso, note que $\rho_{C} \in[0, \theta / 4]$, se $\theta>0$, e $\rho_{C} \in[\theta / 4,0]$, se $\theta<0$.

Na parte superior da Figura 3.1 tem-se o gráfico e as curvas de nível da função Spearman dada por (3.4) para $\theta=0.5$ e na parte inferior para $\theta=-0.75$.
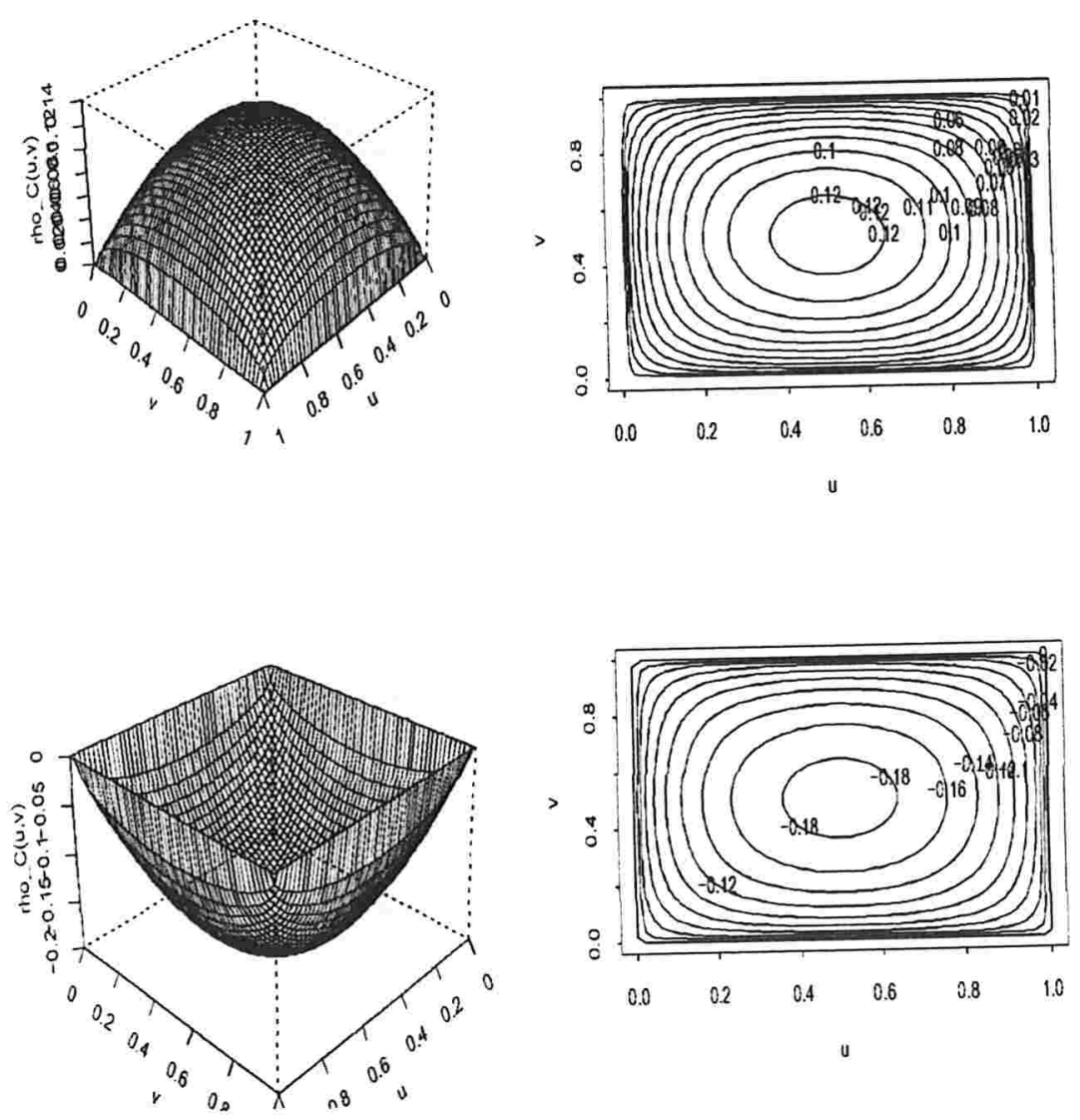

Figura 3.1: Gráfico da função Spearman $\rho_{C}$ e curvas de nível para a família de cópulas FGM. 
Observação 3.3. Do Exemplo 3.1 note que para $\theta>0$ tem-se $\rho_{C}(u, v) \geq 0$ e para $\theta<0$ tem-se $\rho_{C}(u, v) \leq 0$ para todo $(u, v) \in[0,1]^{2}$. Deste modo, podemos melhorar os limites inferior ou superior de Fréchet. Em geral se $\rho_{C}(u, v) \geq 0$ para todo $(u, v) \in[0,1]^{2}$, então

$$
u v \leq C(u, v) \leq \min (u, v)
$$

pela Proposição 3.1. Analogamente, se $\rho_{C}(u, v) \leq 0$, então

$$
\max (u+v-1,0) \leq C(u, v) \leq u v
$$

Note também que a condição $\rho_{C}(u, v) \geq 0$ implica que $C$ é "positive quadrante dependent $(P Q D)$ " de modo análogo $\rho_{C}(u, v) \leq 0$ implica que $C$ é "negative quadrante dependent (NQD)", veja Joe (1997, p. 20)

Exemplo 3.2. Seja C um membro da familia de cópulas de Fréchet (Mardia), i.e. $C(u, v)=a M(u, v)+(1-a-b) u v+b W(u, v)$, em que $M(u, v)=\min (u, v)$, $W(u, v)=\max (u+v-1,0)$ e os coeficientes $a$ e $b$ satisfazem $a \geq 0, b \geq 0 e$ $a+b \leq 1$, veja Nelsen (1999, p. 12). Da relação (3.1) obtemos a seguinte função Spearman associada tem-se que,

$$
\begin{aligned}
\rho_{C}(u, v) & =\frac{a M(u, v)+(1-a-b) u v+b W(u, v)-u v}{\sqrt{u v(1-u)(1-v)}} \\
& =\frac{a M(u, v)-a u v}{\sqrt{u v(1-u)(1-v)}}+\frac{b W(u, v)-b u v}{\sqrt{u v(1-u)(1-v)}} \\
& =a \rho_{M}(u, v)+b \rho_{W}(u, v)
\end{aligned}
$$

em que

$$
\rho_{W}(u, v)=-\min \left(\sqrt{\frac{u v}{(1-u)(1-v)}}, \sqrt{\frac{(1-u)(1-v)}{u v}}\right)
$$

e

$$
\rho_{M}(u, v)=\min \left(\sqrt{\frac{u(1-v)}{v(1-u)}}, \sqrt{\frac{v(1-u)}{u(1-v)}}\right)
$$

são as funções Spearman associadas com os limites inferior e superior de Fréchet, respectivamente. 

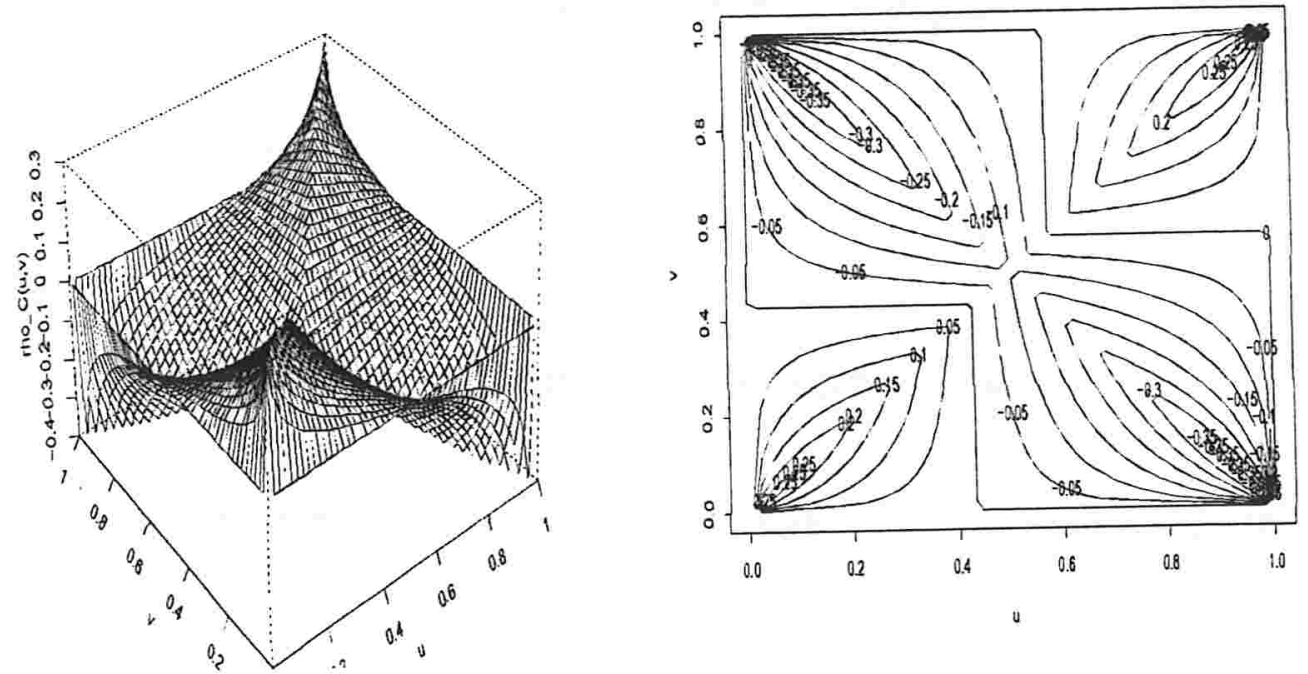

Figura 3.2: Gráfico da função Spearman $\rho_{C}$ e curvas de nível para a família de cópulas de Fréchet.

Na Figura 3.2 são apresentados o gráfico e as curvas de nível da função Spearman do Exemplo 3.2 para $a=0.3$ e $b=0.4$. Note que para esta família tem-se uma dependência positiva para $(u, v) \in\left[0, \frac{a}{a+b}\right]^{2} \cup\left[\frac{b}{a+b}, 1\right]^{2}$ e uma dependência negativa no espaço restante.

Observação 3.4. O Exemplo 3.2 poderia ser estendido mostrando que se $C$ é uma combinação convexa de cópulas então o mesmo é verdadeiro para $\rho_{C}$.

Observação 3.5. Um dos problemas relacionados em Kotz e Nadarajah (2003) com a medida $\gamma_{S}$ é a dificuldade em se encontrar uma forma analítica fechada na maioria dos casos além de não oferecer um representação explícita para cópulas. A função Spearman $\rho_{C}$, como pode-se notar, contorna estes dois problemas.

Portanto, precisamos agora fornecer as condições para que uma função $\rho_{C}$ : $[0,1]^{2} \mapsto[0,1]$ seja uma função Spearman associada a uma cópula $C$. 


\subsection{Caracterização e propriedades da função Spearman}

Nesta seção apresentamos as condições necessárias e suficientes para que uma função seja uma função Spearman e estudamos suas propriedades.

\subsubsection{Caracterização da função Spearman}

Proposição 3.2. Uma função $\rho_{C}:[0,1]^{2} \rightarrow[-1,1]$ é uma função Spearman associada a cópula $C$, se e somente se, ela satisfaz as seguintes condições:

(c1) $\rho_{C}(0, v)=\rho_{C}(u, 0)=0$

(c2) $\rho_{C}(1, v)=\rho_{C}(u, 1)=0$

(c3) Para todo $\left(u_{1}, v_{1}\right),\left(u_{2}, v_{2}\right) \in[0,1]^{2}$ tal que $u_{1} \leq u_{2}$ e $v_{1} \leq v_{2}$, tem-se

$$
\begin{aligned}
& \left(\rho_{C}\left(u_{2}, v_{2}\right) \sqrt{u_{2} v_{2}\left(1-u_{2}\right)\left(1-v_{2}\right)}-\rho_{C}\left(u_{2}, v_{1}\right) \sqrt{u_{2} v_{1}\left(1-u_{2}\right)\left(1-v_{1}\right)}\right)- \\
& \left(\rho_{C}\left(u_{1}, v_{2}\right) \sqrt{u_{1} v_{2}\left(1-u_{1}\right)\left(1-v_{2}\right)}-\rho_{C}\left(u_{1}, v_{1}\right) \sqrt{u_{1} v_{1}\left(1-u_{1}\right)\left(1-v_{1}\right)}\right) \geq \\
& \left(u_{1}-u_{2}\right)\left(v_{2}-v_{1}\right)
\end{aligned}
$$

Prova: Para (c1) observe que,

$$
\rho_{C}(0, v)=\frac{C(0, v)-0}{0}=\frac{0}{0} \quad e \quad \rho_{C}(u, 0)=\frac{C(u, 0)-0}{0}=\frac{0}{0} .
$$

Assim, pela propriedade (p2) na página 8 e utilizando a regra de l'Hospital, obtém-

se

$$
\lim _{u \rightarrow 0} \rho_{C}(u, v)=\frac{2\left[\frac{\partial C(u, v)}{\partial u}-v\right] \sqrt{u v(1-u)(1-v)}}{v(1-v)(1-2 u)}=0
$$

e 


$$
\lim _{v \rightarrow 0} \rho_{C}(u, v)=\frac{2\left[\frac{\partial C(u, v)}{\partial v}-u\right] \sqrt{u v(1-u)(1-v)}}{u(1-u)(1-2 v)}=0 .
$$

Do mesmo modo, pela propriedade (p2) na página 8 e utilizando a regra de l'Hospital, tem-se para (c2) que

$$
\begin{aligned}
& \lim _{u \rightarrow 1} \rho_{C}(u, v)=\frac{2\left[\frac{\partial C(u, v)}{\partial u}-v\right] \sqrt{u v(1-u)(1-v)}}{v(1-v)(1-2 u)}=0 \\
& \lim _{v \rightarrow 1} \rho_{C}(u, v)=\frac{2\left[\frac{\partial C(u, v)}{\partial v}-u\right] \sqrt{u v(1-u)(1-v)}}{u(1-u)(1-2 v)}=0 .
\end{aligned}
$$

e

Para verificar $(\mathbf{c 3})$ note primeiro que para todo $\left(u_{1}, v_{1}\right),\left(u_{2}, v_{2}\right) \in[0,1]^{2}$ tal que $u_{1} \leq u_{2}$ e $v_{1} \leq v_{2}$, tem-se que,

$$
\left(C\left(u_{2}, v_{2}\right)-C\left(u_{2}, v_{1}\right)\right)-\left(C\left(u_{1}, v_{2}\right)-C\left(u_{1}, v_{1}\right)\right) \geq 0
$$

Pela proposição 3.1 e a equação 3.5 , tem-se

$$
\begin{aligned}
& \left(u_{2} v_{2}+\rho_{C}\left(u_{2}, v_{2}\right) \sqrt{u_{2} v_{2}\left(1-u_{2}\right)\left(1-v_{2}\right)}-u_{2} v_{1}-\rho_{C}\left(u_{2}, v_{1}\right) \sqrt{u_{2} v_{1}\left(1-u_{2}\right)\left(1-v_{1}\right)}\right)- \\
& \left(u_{1} v_{2}+\rho_{C}\left(u_{1}, v_{2}\right) \sqrt{u_{1} v_{2}\left(1-u_{1}\right)\left(1-v_{2}\right)}-u_{1} v_{1}-\rho_{C}\left(u_{1}, v_{1}\right) \sqrt{u_{1} v_{1}\left(1-u_{1}\right)\left(1-v_{1}\right)}\right) \geq 0 .
\end{aligned}
$$

Da expressão acima, pode-se concluir para todo $\left(u_{1}, v_{1}\right),\left(u_{2}, v_{2}\right) \in[0,1]^{2}$ tal que $u_{1} \leq u_{2}$ e $v_{1} \leq v_{2}$ que,

$$
\begin{aligned}
& \left(\rho_{C}\left(u_{2}, v_{2}\right) \sqrt{u_{2} v_{2}\left(1-u_{2}\right)\left(1-v_{2}\right)}-\rho_{C}\left(u_{2}, v_{1}\right) \sqrt{u_{2} v_{1}\left(1-u_{2}\right)\left(1-v_{1}\right)}\right)- \\
& \left(\rho_{C}\left(u_{1}, v_{2}\right) \sqrt{u_{1} v_{2}\left(1-u_{1}\right)\left(1-v_{2}\right)}-\rho_{C}\left(u_{1}, v_{1}\right) \sqrt{u_{1} v_{1}\left(1-u_{1}\right)\left(1-v_{1}\right)}\right) \geq \\
& \left(u_{1}-u_{2}\right)\left(v_{2}-v_{1}\right)
\end{aligned}
$$

A volta deste resultado é a seguinte: Seja $\rho_{C}$ uma função satisfazendo as condições (c1), (c2) e (c3), então existe uma cópula $C$ associada a $\rho_{C}$ que satisfaz a seguinte relação para todo $(u, v) \in[0,1]^{2}$,

$$
C(u, v)=u v+\rho_{C}(u, v) \sqrt{u v(1-u)(1-v)} .
$$


De fato, note que a função $C$ definida acima é uma cópula, pois

1. Da condição (c1) segue que,

$$
\begin{array}{r}
C(0, v)=0 v+\underbrace{\rho_{C}(0, v)}_{=0} \sqrt{0 v(1-0)(1-v)}=0 \\
e \quad C(0, v)=u 0+\underbrace{\rho_{C}(u, 0)}_{=0} \sqrt{u 0(1-u)(1-0)}=0
\end{array}
$$

2. Da condição (c2) tem-se,

$$
\begin{array}{r}
C(1, v)=v+\underbrace{\rho_{C}(1, v)}_{=0} \sqrt{v(1-1)(1-v)}=v \\
e \quad C(0, v)=u+\underbrace{\rho_{C}(u, 1)}_{=0} \sqrt{u(1-u)(1-1)}=u
\end{array}
$$

3. Finalmente da condição (c3) segue-se que,

$$
\begin{array}{r}
{\left[C\left(u_{2}, v_{2}\right)-C\left(u_{2}, v_{1}\right)\right)-\left(C\left(u_{1}, v_{2}\right)-C\left(u_{1}, v_{1}\right)\right]=} \\
{\left[u_{2} v_{2}+\rho_{C}\left(u_{2}, v_{2}\right) \sqrt{u_{2} v_{2}\left(1-u_{2}\right)\left(1-v_{2}\right)}-\right.} \\
\left.u_{2} v_{1}-\rho_{C}\left(u_{2}, v_{1}\right) \sqrt{u_{2} v_{1}\left(1-u_{2}\right)\left(1-v_{1}\right)}\right]- \\
{\left[u_{1} v_{2}+\rho_{C}\left(u_{1}, v_{2}\right) \sqrt{u_{1} v_{2}\left(1-u_{1}\right)\left(1-v_{2}\right)}-\right.} \\
\left.u_{1} v_{1}-\rho_{C}\left(u_{1}, v_{1}\right) \sqrt{u_{1} v_{1}\left(1-u_{1}\right)\left(1-v_{1}\right)}\right]= \\
{\left[\rho_{C}\left(u_{2}, v_{2}\right) \sqrt{u_{2} v_{2}\left(1-u_{2}\right)\left(1-v_{2}\right)}-\rho_{C}\left(u_{2}, v_{1}\right) \sqrt{u_{2} v_{1}\left(1-u_{2}\right)\left(1-v_{1}\right)}\right]-} \\
{\left[\rho_{C}\left(u_{1}, v_{2}\right) \sqrt{u_{1} v_{2}\left(1-u_{1}\right)\left(1-v_{2}\right)}-\rho_{C}\left(u_{1}, v_{1}\right) \sqrt{u_{1} v_{1}\left(1-u_{1}\right)\left(1-v_{1}\right)}\right]+} \\
{\left[\rho_{C}\left(u_{2}, v_{2}\right) \sqrt{u_{2} v_{2}\left(1-u_{2}\right)\left(1-v_{1}\right)-\left(u_{1} v_{2}-u_{1} v_{1}\right)}-\rho_{C}\left(u_{2}, v_{1}\right) \sqrt{u_{2} v_{1}\left(1-u_{2}\right)\left(1-v_{1}\right)}\right]-} \\
{\left[\rho_{C}\left(u_{1}, v_{2}\right) \sqrt{u_{1} v_{2}\left(1-u_{1}\right)\left(1-v_{2}\right)}-\rho_{C}\left(u_{1}, v_{1}\right) \sqrt{u_{1} v_{1}\left(1-u_{1}\right)\left(1-v_{1}\right)}\right] \geq 0} \\
\text { para todo }\left(u_{1}, v_{1}\right),\left(u_{2}, v_{2}\right) \in[0,1]^{2} \text { tal } \text { que } u_{1} \leq u_{2} \text { e } v_{1} \leq v_{2} .
\end{array}
$$


Portanto, como a função $C$ definida por $\rho_{C}$ é uma cópula, segue que $\rho_{C}$ é a função Spearman associada a $C$.

Conseqüentemente, para toda função $\rho_{C}:[0,1]^{2} \mapsto[-1,1]$ que satisfaça as condições da Proposição 3.2 tem-se uma cópula $C$ dada pela Proposição 3.1 associada e por conseguinte uma família de função distribuição conjunta $H$ com marginais $F$ e $G$ associada a $\rho_{H}$.

A condição (c3) da Proposição 3.2 pode ser trocada por

$$
\frac{\partial^{2}}{\partial u \partial v} \rho_{C}(u, v) \sqrt{u v(1-u)(1-v)} \geq-1
$$

se existir $\frac{\partial^{2} C(u, v)}{\partial u \partial v}>0$. Esta condição é mais simples de ser verificada, como demonstraremos nos exemplos seguintes.

Exemplo 3.3 (continuação Exemplo 3.1). No Exemplo 3.1 obtemos a função Spearman associada com a cópula da familia FGM. É fácil ver que as condições (c1) e (c2) da Proposição 3.2 são satisfeitas. A condição (3.6) é dada por,

$$
\frac{\partial^{2}}{\partial u \partial v} \rho_{C}(u, v) \sqrt{u v(1-u)(1-v)}=\theta(1-2 u)(1-2 v) \geq-1
$$

para todo $(u, v) \in[0,1]^{2}$ e $|\theta| \leq 1$.

Exemplo 3.4. Considere a seguinte função,

$$
\rho_{C}(u, v)=\theta[u v(1-u)(1-v)]^{\alpha} \quad \text { para } \quad \alpha \in[0.5, \infty) .
$$

Neste caso tem-se que $\rho_{C}(u, 0)=\rho_{C}(0, v)=0, \rho_{C}(u, 1)=\rho_{C}(1, v)=0 e$

$$
\begin{aligned}
\frac{\partial^{2}}{\partial u \partial v} \rho_{C}(u, v) \sqrt{u v(1-u)(1-v)} & =\frac{\partial^{2}}{\partial u \partial v}\left[\theta[u v(1-u)(1-v)]^{\alpha+1 / 2}\right] \\
& =\frac{\theta[u v(1-u)(1-v)]^{\alpha-1 / 2}}{4}(2 \alpha+1)^{2}(1-2 v)(1-2 u)
\end{aligned}
$$


que é maior que - 1 para todo $(u, v) \in[0,1]^{2}$ para

$$
|\theta| \leq \max _{(u, v) \in[0,1]^{2}}\left|\frac{(2 \alpha+1)^{2}}{4}[u v(1-u)(1-v)]^{\alpha-1 / 2}(1-2 v)(1-2 u)\right| .
$$

\subsubsection{Propriedades da função Spearman}

Apresentamos algumas propriedades da função Spearman em função da cópula associada. Note que as propriedades das medidas de dependência global vistas na Seção 2.4.2 diferem um pouco das propriedades de medidas de dependência locais, pois estas propriedades são essencialmente locais.

(p1) Para toda função $\rho_{C}$ e todo $\operatorname{par}(u, v) \in[0,1]^{2}$ tem-se:

$$
\rho_{W}(u, v) \leq \rho_{C}(u, v) \leq \rho_{M}(u, v)
$$

em que $\rho_{W}(u, v)$ e $\rho_{M}(u, v)$ são as funções Spearman associadas aos limites de Fréchet $W(u, v)=\max (u+v-1,0)$ e $M(u, v)=\min (u, v)$ dadas no Exemplo 3.2

(p2) $\rho_{C}(u, v)=0$ para todo $\operatorname{par}(u, v) \in[0,1]^{2}$, se e somente se a cópula associada é a cópula da independência;

(p3) $\rho_{C}$ é simétrica se e somente se $C$ for simétrica, i.e.,

$$
\rho_{C}(u, v)=\rho_{C}(v, u) \Longleftrightarrow C(u, v)=C(v, u)
$$

(p4) Denotando por $\rho_{C_{X, Y}}$ a função Spearman associada a estrutura de dependència das variáveis aleatórias $X$ e $Y$. Seja $\alpha$ e $\beta$ funções estritamente crescente e decrescente respectivamente, no suporte de $X$ então,

$$
\rho_{C_{\alpha(X), Y}}(u, v)=\rho_{C_{X, Y}}(u, v)
$$


e

$$
\rho_{C_{\beta(X), Y}}(u, v)=-\rho_{C_{X, Y}}(1-u, v)
$$

(p5) Como $\rho_{C}$ é igual ao coeficiente de correlação linear entre as variáveis indicadoras $\mathbf{I}\{U \leq u\}$ e $\mathbf{I}\{V \leq v\}$ para todo $(u, v) \in[0,1]^{2}$, e portanto $\rho_{C} \in[-1,1]$.

Prova: As propriedades (p1) a (p4) são resultados simples que decorrem diretamente da Proposição 3.1 e das propriedades das cópulas.

\subsection{Representação para cópulas via cópula gaussiana local}

Nesta seção apresentamos uma nova medida de dependência local $r(u, v)$ que pode ser interpretada como uma medida da distância entre uma cópula $C$ e a cópula gaussiana $\Phi_{r}$ e exibimos algumas propriedades.

Proposição 3.3. Seja C uma cópula e seja $\Phi_{r}\left(\Phi^{-1}(u), \Phi^{-1}(v)\right)$ a cópula gaussiana com coeficiente de correlação linear $r$. Então para cada par $(u, v) \in[0,1]^{2}$ existe um único valor,

$$
r(u, v)=\inf \left\{r: \Phi_{r}\left(\Phi^{-1}(u), \Phi^{-1}(v)\right) \geq C(u, v)\right\} \in[-1,1],
$$

tal que

$$
C(u, v)=\Phi_{r(u, v)}\left(\Phi^{-1}(u), \Phi^{-1}(v)\right) .
$$

Prova: Para provar esta proposição é suficiente mostrar que para toda cópula $C$ e todo par $(u, v) \in[0,1]^{2}$ existe $r \in[-1,1]$ tal que $C(u, v)=\Phi_{r}\left(\Phi^{-1}(u), \Phi^{-1}(v)\right)$.

De fato, primeiro note que,

$$
C(0, v)=\Phi_{r}\left(\Phi^{-1}(0), \Phi^{-1}(v)\right)=C(u, 0)=\Phi_{r}\left(\Phi^{-1}(u), \Phi^{-1}(0)\right)=0
$$


e

$$
C(1, v)=\Phi_{r}\left(\Phi^{-1}(1), \Phi^{-1}(v)\right)=v \quad e \quad C(u, 1)=\Phi_{r}\left(\Phi^{-1}(u), \Phi^{-1}(1)\right)=u
$$

para todo $(u, v) \in[0,1]^{2}$ e $r \in[-1,1]$.

Agora, fixe $(u, v) \in(0,1)^{2}$, então,

$$
\Phi_{r}\left(\Phi^{-1}(u), \Phi^{-1}(v)\right)=\int_{\Phi^{-1}(u)}^{\infty} \int_{\Phi^{-1}(v)}^{\infty} \frac{1}{2 \pi \sqrt{1-r^{2}}} \exp \left\{-\frac{\xi^{2}-2 r \xi \varsigma+\varsigma^{2}}{2\left(1-r^{2}\right)}\right\} d \varsigma d \xi
$$

é uma função contínua para $r \in(-1,1) e$

$$
\lim _{r \rightarrow-1} \Phi_{r}\left(\Phi^{-1}(u), \Phi^{-1}(v)\right)=\max (u+v-1,0)
$$

$e$

$$
\lim _{r \rightarrow 1} \Phi_{r}\left(\Phi^{-1}(u), \Phi^{-1}(v)\right)=\min (u, v)
$$

Agora, a partir dos fatos acima e do fato que

$$
\max (u+v-1,0) \leq C(u, v) \leq \min (u, v),
$$

para todo $(u, v) \in[0,1]^{2}$, segue que existe $r \in[-1,1]$ tal que $C(u, v)=\Phi_{r}\left(\Phi^{-1}(u), \Phi^{-1}(v)\right)$ para toda cópula $C$ e todo par $(u, v) \in[0,1]^{2}$.

Observação 3.6. Note que a Proposição 3.3 não afirma que toda cópula C é uma cópula gaussiana, mas que para cada par $(u, v)$ sempre existe um membro da familia gaussiana com coeficiente de correlação $r=r(u, v)$ tal que (3.8) é sempre válido. Note que, $r(u, v)$ definido em (3.7) é também uma medida de dependência local que está relacionada com o afastamento entre a cópula $C$ e a cópula gaussiana, de tal forma que quando uma cópula for um membro da familia gaussiana com coeficiente de correlação $r$ então $r(u, v)=r$ caso contrário $r(u, v)$ será uma função de $(u, v)$. $\square$ 


\subsubsection{Propriedades}

A seguir damos algumas propriedade de $r(u, v)$, observe que algumas são iguais às da função Spearman $\rho_{C}$, veja Seção 3.2.2.

(p1) Para toda função $r(u, v)$ e todo par $(u, v) \in[0,1]^{2}$ tem-se:

$$
-1 \leq r(u, v) \leq 1,
$$

esses casos limites estão associados aos limites de Fréchet $W(u, v)=\max (u+$ $v-1,0)$ e $M(u, v)=\min (u, v)$, veja a prova da Proposição 3.3;

(p2) $r(u, v)=0$ para todo par $(u, v) \in[0,1]^{2}$, se e somente se a cópula associada a $r(u, v)$ é a cópula da independência;

(p3) $r(u, v)=r$ para todo par $(u, v) \in[0,1]^{2}$, se e somente se a cópula associada a $r(u, v)$ é um membro da família de cópulas gaussiana com coeficiente de correlação $r$;

(p4) $r(u, v)$ é simétrica se e somente se a cópula associada $C$ for simétrica, i.e.,

$$
r(u, v)=r(v, u) \Longleftrightarrow C(u, v)=C(v, u)
$$

(p5) Denotando por $r_{C_{X, Y}}(u, v)$ a função $r(u, v)$ associada a estrutura de dependència das variáveis aleatórias $X$ e $Y$. Seja $\alpha$ e $\beta$ funções estritamente crescente e decrescente respectivamente, no suporte de $X$ então,

$$
r_{C_{\alpha(X), Y}}(u, v)=r_{C_{X, Y}}(u, v)
$$

e

$$
r_{C_{B(X), \beta(Y)}}(u, v)=r_{\bar{C}_{X, Y}}(u, v)
$$

em que $\bar{C}_{X, Y}$ é a cópula da sobrevivência; 
Prova: As propriedades (p1) a (p5) são resultados simples que decorrem diretamente da Proposição 3.3 e das propriedades das cópulas.

Neste capítulo apresentamos duas novas medidas de dependência locais sendo que a função Spearman tem uma simples representação em termos de cópulas e a outra que pode ser interpretada como um medida de dependência local que mede a distancia entre uma cópula $C$ e a cópula gaussiana $\Phi_{r}$. Todos estes resultados podem também ser encontrados em Anjos e Kolev (2005b) e Kolev et al. (2005). 


\section{Capítulo 4}

\section{Cópula para estatísticas de ordem de vetores bivariados}

Uma das estruturas de dependência que tem particular importància na estatística é a estrutura de dependência das estatística de ordem. Entretanto, na literatura não existem muitos resultados sobre estatísticas de ordem e sua cópula associada. Por exemplo em Nelsen (1999, p. 25) encontramos os seguintes resultados para as estatísticas de ordem $\max (X, Y)$ e $\min (X, Y)$ do vetor aleatório $(X, Y)$,

$$
P(\max (X, Y) \leq t)=C(F(t), G(t))
$$

e

$$
P(\min (X, Y) \leq t)=F(t)+G(t)-C(F(t), G(t)) .
$$

A relação acima é generalizada em George et al. (2001) como segue: Seja $\left(X_{1}, \ldots, X_{n}\right)$ ım vetor aleatório com $F_{i}(x)=P\left(X_{i} \leq x\right)$ contínua para $i=1, \ldots, n$ e cópula associada $C_{n}$. Seja $X_{r: n}$ a $r$-ésima estatística de ordem $(1 \leq r \leq n)$ do vetor $\left(X_{1}, \ldots, X_{n}\right)$. Então a função distribuição $F_{r: n}(t)=P\left(X_{r: n} \leq t\right)$ é dada por

$$
F_{r: n}(t)=\sum_{k=r}^{n}\left[\sum_{l=r}^{k}(-1)^{k-l}\left(\begin{array}{l}
k \\
l
\end{array}\right) \sum C_{n}\left(F_{1}(t), \ldots, F_{n}(t)\right)\right],
$$


em que $\sum$ denota a somatória sobre o conjunto

$$
\left\{\left(v_{1}, \ldots, v_{n}\right) \in[0,1]^{n}: v_{i} \in\left\{F_{i}(t), 1\right\}, \sum_{i=1}^{n} \delta_{\{1\}}\left(v_{i}\right)=n-k\right\}
$$

$\operatorname{com} \delta_{\{1\}}\left(v_{i}\right)=1$ se $v_{i}=1$, e 0 caso contrário. Da relação (4.1) tem-se que

$$
F_{1: n}(t)=1-\overline{C_{n}}\left(S_{1}(t), \ldots, S_{n}(t)\right)
$$

onde $\overline{C_{n}}$ é a cópula da sobrevivència e $S_{i}(t)=1-F_{i}(t)$. Note também que a função distribuição $F_{n: n}$ de $X_{n: n}=\max \left(X_{1}, \ldots, X_{n}\right)$ é a seção diagonal da cópula $C_{n}$ associada ao vetor $\left(X_{1}, \ldots, X_{n}\right)$, i.e..

$$
F_{n: n}(t)=C_{n}\left(F_{1}(t), \ldots, F_{n}(t)\right)
$$

Pode-se também estar interessado em outras estatísticas que são relevantes em confiabilidade, análise de sobrevivência ou análise de risco. Por exemplo, poderíamos estar interessados em $X_{n: n}-X_{1: n}$ ou $X_{r_{1}: n}-X_{r_{2}: n}$ para $r_{1}>r_{2}$. Entretanto, para que possamos estudar tais estatísticas precisamos da distribuição conjunta de $X_{r_{1}: n}$ e $X_{r_{2}: n}$. No caso em que as variáveis são independentes e identicamente distribuídas Balakrishnan e Cohen (1991) fornecem expressões mais amigáveis para a densidade. Nelsen (2003) apresenta a cópula $C_{1, n}$ para $X_{1: n}$ e $X_{n: n}$

$$
C_{1, n}(u, v)=v-\left[\max \left\{(1-u)^{\frac{1}{n}}+v^{\frac{1}{n}}-1,0\right\}\right]^{n} .
$$

No caso geral, uma solução é usar a simulação de Monte Carlo como sugerido em George et al. (2001). Recentemente, Avérous et al. (2005) estudaram o grau de associação entre as estatísticas de ordem de vetores bivariados.

Neste capítulo apresentamos alguns resultados envolvendo estatísticas de ordem e sua cópula associada. Na Seção 4.1 mostramos a cópula para a r-ésima e $s$-ésima estatísticas de ordem do vetor $(X, Y)$ em uma amostra aleatória de tamanho $n$. Na 
Seção 4.2 apresentamos para o caso em que $r / n \rightarrow \lambda_{1}$ e $s / n \rightarrow \lambda_{2}$ quando $n \rightarrow \infty$ tal que $0 \leq \lambda_{1}, \lambda_{2}<1$ ou $\lambda_{1}=0$ e $\lambda_{2}=1$. Na Seção 4.3 mostramos uma relação de recorrência para as cópulas de estatísticas de ordem apresentadas na Seção 4.1. Finalmente, na Seção 4.4 apresentamos os limites de Fréchet relacionados com estas cópulas. Os resultados apresentados são ilustrados com alguns exemplos numéricos.

\subsection{Cópula para estatísticas de ordem}

Seja $\left\{\left(X_{1}, Y_{1}\right), \ldots,\left(X_{n}, Y_{n}\right)\right\}$ uma amostra aleatória do vetor $(X, Y)$, com funções distribuição marginais $F$ e $G$ contínuas, função distribuição conjunta $H$ e cópula associada $C$. Seja $\left\{X_{1: n}, \ldots, X_{n: n}\right\}$ e $\left\{Y_{1: n}, \ldots, Y_{n: n}\right\}$ as estatísticas de ordem associada a amostra. Nesta seção apresentamos a cópula $C_{X_{r: n}, Y_{s: n}}$ associada com as estatísticas de ordem $X_{r: n}$ e $Y_{s: n}$, como segue.

Denotando,

$$
R_{x}=\sum_{j=1}^{n} I\left\{X_{j} \leq x\right\} \quad \text { e } \quad R_{y}=\sum_{j=1}^{n} I\left\{Y_{j} \leq y\right\},
$$

para todo par $(x, y) \in[-\infty, \infty]^{2}$, em que $I\{A\}$ é a função indicadora do conjunto A. Portanto, $R_{x} \sim \operatorname{Bin}(n, p)$ e $R_{y} \sim \operatorname{Bin}(n, q), \operatorname{com} p=P(X \leq x)=F(x)$ e $q=P(Y \leq y)=G(y)$.

Fazendo a transformação $\left(F\left(X_{i}\right), G\left(Y_{i}\right)\right)$ em cada coordenada da amostra aleatória $\left\{\left(X_{1}, Y_{1}\right), \ldots,\left(X_{n}, Y_{n}\right)\right\}$, obtém-se $\left\{\left(U_{1}, V_{1}\right), \ldots,\left(U_{n}, V_{n}\right)\right\}$, que pela transformada integral da probabilidade sabemos que $U_{i} \sim U(0,1)$ e $V_{i} \sim U(0,1)$ para todo $i=1 \ldots n$, pois $F$ e $G$ são contínuas. Agora note que, fixos $x, y, r$ e $s$ tem-se para todo $\omega \in \Omega$ que,

$$
\left\{\omega: R_{x}(\omega) \geq r\right\}=\left\{\omega: X_{r: n}(\omega) \leq x\right\} \quad \text { e } \quad\left\{\omega: R_{y}(\omega) \geq s\right\}=\left\{\omega: X_{s: n}(\omega) \leq y\right\} .
$$


Conseqüientemente,

$$
\left\{\omega: R_{x}(\omega) \geq r, R_{y}(\omega) \geq s\right\}=\left\{\omega: X_{r: n}(\omega) \leq u, Y_{s: n}(\omega) \leq v\right\}
$$

Como a transformações $F\left(X_{i}\right)$ e $G\left(Y_{i}\right)$ são monótonas não decrescentes, tem-se para todo $(u, v) \in[0,1]^{2}$ para o qual $u=F(x)$ e $v=G(y)$,

$$
\begin{aligned}
& R_{u}(\omega)=\sum_{j=1}^{n} I\left\{U_{j}(\omega) \leq u\right\}=\sum_{j=1}^{n} I\left\{X_{j}(\omega) \leq x\right\}=R_{x}(\omega) \\
& R_{v}(\omega)=\sum_{j=1}^{n} I\left\{V_{j}(\omega) \leq v\right\}=\sum_{j=1}^{n} I\left\{Y_{j}(\omega) \leq y\right\}=R_{y}(\omega)
\end{aligned}
$$

para todo $\omega \in \Omega$. Portanto,

$$
\left\{\omega: R_{u}(\omega) \geq r, R_{v}(\omega) \geq s\right\}=\left\{\omega: R_{x}(\omega) \geq r, R_{y}(\omega) \geq s\right\}
$$

Denotando por $\left\{U_{1: n}, \ldots, U_{n: n}\right\}$ e $\left\{V_{1: n}, \ldots, V_{n: n}\right\}$ as estatística de ordem da amostra aleatória $\left\{\left(U_{1}, V_{1}\right), \ldots,\left(U_{n}, V_{n}\right)\right\}$ das variáveis $U_{i}=F\left(X_{i}\right)$ e $V_{i}=G\left(Y_{i}\right)$. tem-se de modo análogo a relação (4.2) que,

$$
\left\{\omega: R_{u}(\omega) \geq r\right\}=\left\{\omega: U_{r: n}(\omega) \leq u\right\} \quad \text { e } \quad\left\{\omega: R_{v}(\omega) \geq s\right\}=\left\{\omega: V_{s: n}(\omega) \leq v\right\}
$$

conseqüentemente, fixos $u=F(x), v=G(y), r$ e $s$ tem-se,

$$
\left\{\omega: R_{u}(\omega) \geq r, R_{v}(\omega) \geq s\right\}=\left\{\omega: U_{r: n}(\omega) \leq u, V_{s: n}(\omega) \leq v\right\}
$$

para todo $\omega \in \Omega$. Nestas condições, segue das relações (4.3), (4.4) e (4.5) que,

$$
P\left(X_{r: n} \leq x, Y_{s: n} \leq y\right)=P\left(R_{F(x)} \geq r, R_{G(y)} \geq s\right)=P\left(U_{r: n} \leq F(x), V_{s: n} \leq G(y)\right)
$$

on de modo semelhante,

$$
P\left(X_{r: n} \leq F^{-1}(u), Y_{s: n} \leq G^{-1}(v)\right)=P\left(R_{u} \geq r, R_{v} \geq s\right)=P\left(U_{r: n} \leq u, V_{s: n} \leq v\right)
$$


Portanto, para $u=F(x)$ e $v=G(y)$ a seguinte relação é válida

$$
H_{X_{r: n}, Y_{s: n}}(x, y)=H_{U_{r: n}, V_{s: n}}(F(x), G(y))
$$

o que implica $C_{X_{r: n}, Y_{s: n}}=C_{U_{r: n}, V_{s: n}}$.

A função geradora de probabilidades para $\left(R_{u}, R_{v}\right)$ é dada por

$$
\begin{aligned}
\Pi_{R_{u}, R_{v}}\left(t_{1}, t_{2}\right) & =E\left(t_{1}^{R_{u}} t_{2}^{R_{v}}\right) \\
& =\left[E\left(t_{1}^{I\left\{U_{j} \leq u\right\}} t_{2}^{I\left\{V_{j} \leq v\right\}}\right)\right]^{n} \quad \text { amostra i.i.d. } \\
& =\left[1+u\left(t_{1}-1\right)+v\left(t_{2}-1\right)+C(u, v)\left(t_{1}-1\right)\left(t_{2}-1\right)\right]^{n} .
\end{aligned}
$$

Portanto a função distribuição conjunta de $\left(R_{u}, R_{v}\right)$ é uma binomial bivariada. O coeficiente de correlação entre $R_{u}$ e $R_{v}$ é dado por.

$$
\rho_{R_{u}, R_{v}}=\frac{C(u, v)-u v}{\sqrt{u v(1-v)(1-v)}}=\rho_{C}(u, v)
$$

Portanto o coeficiente de correlação entre $R_{u}$ e $R_{v}$ é igilal a função Spearman associada a cópula $C$.

Sendo assim, a função distribuição conjunta de $\left(U_{r: n}, V_{s: n}\right)$ é dada por

$$
\begin{aligned}
P\left(U_{r: n} \leq u, V_{s: n} \leq v\right) & =P\left(R_{u} \geq r, R_{v} \geq s\right) \\
& =\sum_{j=r}^{n} \sum_{k=s}^{n} \sum_{m} \frac{n ! C(u, v)^{m}(u-C(u, v))^{j-m}(v-C(u, v))^{k-m}}{m !(j-m) !(k-m) !(n-j-k+m) !} \\
& \times(1-u-v+C(u, v))^{n-j-k+m},
\end{aligned}
$$

em que $m$ é o número de pares $\left(U_{i}, V_{i}\right)$ tal que $U_{i} \leq u$ e $V_{i} \leq v, i=1, \ldots n$. com $\max (0, j+k-n) \leq m \leq \min (j, k)$.

Da equação (4.7) pode-se obter as funções geradoras de probabilidade das marginais fazendo $t_{1}=1$ e $t_{2}=1$. Deste modo, para $R_{u}$ tem-se

$$
\Pi_{R_{u} \cdot R_{v}}\left(t_{1}, 1\right)=\Pi_{R_{u}}\left(t_{1}\right)=\left[1+u\left(t_{1}-1\right)^{n}\right.
$$


conseqüentemente

$$
P\left(U_{r: n} \leq u\right)=P\left(R_{u} \geq r\right)=\sum_{j=r}^{n}\left(\begin{array}{l}
n \\
j
\end{array}\right) u^{j}(1-u)^{n-j}
$$

Derivando a equação (4.10) com relação a $u$ obtém-se a densidade de $U_{r: n}$, assim

$$
f_{U_{r: n}}(u)=\frac{d P\left(U_{r: n} \leq u\right)}{d u}=\frac{\Gamma(n+1)}{\Gamma(r) \Gamma(n-r+1)} u^{r-1}(1-u)^{(n-r+1)-1}
$$

Deste modo, $U_{r: n}$ é uma Beta $(r, n-r+1)$. Do mesmo modo, obtém-se que $V_{s: n}$ é ıma $\operatorname{Beta}(s, n-s+1)$. Portanto, as distribuições marginais da distribuição conjunta $P\left(U_{r: n} \leq u, V_{s: n} \leq v\right)$ são distribuições Beta. Do mesmo modo, podemos obter a densidade conjunta de $U_{r: n}, V_{s: n}$, calculando

$$
f_{U_{r: n}, V_{s: n}}(u, v)=\frac{\partial^{2} P\left(U_{r: n} \leq u, V_{s: n} \leq v\right)}{\partial u \partial v}
$$

No entanto o cálculo desta densidade não resulta em nenhuma distribuição conhecida. Da equação (4.9) podemos obter a cópula de $\left(U_{r: n}, V_{s: n}\right)$ e por conseguinte de $\left(X_{r: n}, Y_{s: n}\right)$. Seja $\beta_{r, n-r+1}^{-1}(w)$ e $\beta_{s, n-s+1}^{-1}(t)$ as inversas das distribuições Beta, para $w . t \in[0,1]$, então,

$$
\begin{aligned}
C_{X_{r: n}, Y_{s: n}}(w, t) & =C_{U_{r: n}, V_{s: n}}(w, t)=H_{U_{r: n}, V_{s: n}}\left(\beta_{r, n-r+1}^{-1}(w), \beta_{s, n-s+1}^{-1}(t)\right) \\
& =\sum_{j=r}^{n} \sum_{k=s}^{n} \sum_{m} \frac{n ! C\left(\beta_{r, n-r+1}^{-1}(w), \beta_{s, n-s+1}^{-1}(t)\right)^{m}}{n_{u v} !(j-m) !(k-m) !(n-j-k+m) !} \\
& \times\left(\beta_{r, n-r+1}^{-1}(w)-C\left(\beta_{r, n-r+1}^{-1}(w), \beta_{s, n-s+1}^{-1}(t)\right)\right)^{j-m} \\
& \times\left(\beta_{s, n-s+1}^{-1}(t)-C\left(\beta_{r, n-r+1}^{-1}(w), \beta_{s, n-s+1}^{-1}(t)\right)\right)^{k-m} \\
& \times\left(1-\beta_{r, n-r+1}^{-1}(w)-\beta_{s, n-s+1}^{-1}(t)+C\left(\beta_{r, n-r+1}^{-1}(w), \beta_{s, n-s+1}^{-1}(t)\right)\right)^{n-j-k+m} .
\end{aligned}
$$

\subsection{Cópula Assintótica}

Em Barakat (2001) propriedades da distribuição conjunta assintótica de $\left(R_{u}, R_{v}\right)$ sào investigados e como conseqüència são obtidas distribıições limites para o vetor 
$\left(X_{r: n}, Y_{s: n}\right)$ para $1 \leq r, s \leq n$ para cada tipo de taxa de convergència. Ao todo são apresentados dez casos. Nesta seção, consideremos apenas o caso em que $r / n \rightarrow \lambda_{1}$ e $s / n \rightarrow \lambda_{2}$ quando $n \rightarrow \infty$ tal que $0 \leq \lambda_{1}, \lambda_{2}<1$ ou $\lambda_{1}=0, \lambda_{2}=1$, mas o método elaborado aqui pode ser utilizado de maneira similar no outros casos.

A idéia é simples, extraímos a cópula assintótica, que denotaremos por $C^{a}$, associada com a distribuição limite de $\left(R_{u}, R_{v}\right)$ e usamo-la para determinar uma aproximação para a função distribuição conjunta de $\left(X_{r: n}, Y_{s: n}\right)$.

O resultado básico é o Teorema 2.2 em Barakat (2001) que fornece as condições para que tenhamos a convergència em distribuição para a normal bivariada. Considerando as nossas notações o teorema fica da seguinte forma.

Teorema 4.1 (Barakat (2001)). Seja $\min (n-r, r) \rightarrow \infty$ e $\min (n-s, s) \rightarrow \infty$ quando $n \rightarrow \infty$. Além do mais, seja $r / n \rightarrow \lambda_{1}$ es $s \rightarrow \lambda_{2}$ quando $n \rightarrow \infty$ tal que $0 \leq \lambda_{1}, \lambda_{2}<1$ ou $\lambda_{1}=0$ e $\lambda_{2}=1$. Se

$$
\rho_{R_{u}, R_{v}}=\operatorname{Corr}\left(R_{u}, R_{v}\right) \stackrel{n \rightarrow \infty}{\longrightarrow} \rho=\frac{C(u, v)-u v}{\sqrt{u v(1-u)(1-v)}}
$$

para constante $\rho$ tal que $|\rho| \leq 1$,

$$
\frac{r-n u}{\sqrt{n u(1-u)}} \stackrel{n \rightarrow \infty}{\longrightarrow} \tau_{1} \quad \text { e } \frac{s-n v}{\sqrt{n v(1-v)}} \stackrel{n \rightarrow \infty}{\longrightarrow} \tau_{2}
$$

é válida para constante $\tau_{1}$ e $\tau_{2}$ fixas, então

$P\left(\frac{R_{u}-n u}{\sqrt{n u(1-u)}} \leq \frac{r-n u}{\sqrt{n u(1-u)}}, \frac{R_{v}-n v}{\sqrt{n v(1-v)}} \leq \frac{s-n v}{\sqrt{n v(1-v)}}\right) \stackrel{n \rightarrow \infty}{\longrightarrow} \Phi_{\rho}\left(\tau_{1}, \tau_{2}\right)$, em que $\Phi_{\rho}$ é a função distribuição da normal bivariada com coeficiente de correlação $\rho$.

Sobre as condiçōes do Teorema 4.1 e do fato que $C(w, t)=H\left(F^{-1}(w), G^{-1}(t)\right)$. a cópula assintótica $C^{a}$ de $\left(R_{u}, R_{v}\right)$ é dado por

$$
C^{a}(w, t)=\Phi_{\rho}\left(\Phi^{-1}(w), \Phi^{-1}(t)\right)
$$


e a correspondente cópula da sobrevivència

$$
\bar{C}^{a}(w, t)=\bar{\Phi}_{\rho}\left(\bar{\Phi}^{-1}(w), \bar{\Phi}^{-1}(t)\right)
$$

para $w, t \in[0,1]$. Usando a cópula da sobrevivência assintótica $\bar{C}^{a}$ podemos determinar uma aproximação para a função sobrevivència de $\left(R_{u}, R_{v}\right)$. As distribuiçōes marginais de $\left(R_{u}, R_{v}\right)$ e $\left(U_{r: n}, V_{s: n}\right)$ tèm a seguinte relação

$$
P\left(R_{u} \geq r\right)=P\left(U_{r: n} \leq u\right) \quad \text { e } \quad P\left(R_{v} \geq s\right)=P\left(V_{s: n} \leq v\right)
$$

Portanto,

$$
\bar{H}_{R_{u}, R_{v}}(r, s) \approx \bar{\Phi}_{\rho}\left(\bar{\Phi}^{-1}\left(\beta_{r, n-r+1}(u)\right), \bar{\Phi}^{-1}\left(\beta_{s, n-s+1}(v)\right)\right) .
$$

Mas $H_{U_{r: n}, V_{s: n}}(u, v)=\bar{H}_{R_{u}, R_{v}}(r, s)$, assim sobre as condições do Teorema 4.1 segue que

$$
H_{U_{r: n}, V_{s: n}}(u, v) \approx \bar{\Phi}_{\rho}\left(\bar{\Phi}^{-1}\left(\beta_{r, n-r+1}(u)\right), \bar{\Phi}^{-1}\left(\beta_{s, n-s+1}(v)\right)\right) .
$$

Agora utilizando a conhecida relação $\bar{C}(w, t)=w+t-1+C(1-w, 1-t)$ em que $\bar{C}(w, t)$ é a cópula da sobrevivência, tem-se

$$
\begin{aligned}
H_{U_{r: n}, V_{s: n}}(u, v) \approx & \beta_{r, n-r+1}(u)+\beta_{s, n-s+1}(v)-1+ \\
& \Phi_{\rho}\left(\Phi^{-1}\left(1-\beta_{r, n-r+1}(u)\right), \Phi^{-1}\left(1-\beta_{s, n-s+1}(v)\right)\right) .
\end{aligned}
$$

Finalmente, da relação (4.6) obtemos,

$$
\begin{aligned}
H_{X_{r: n}, Y_{s: n}}(x, y) \approx & \beta_{r, n-r+1}(F(x))+\beta_{s, n-s+1}(G(y))-1+ \\
& \Phi_{\rho}\left(\Phi^{-1}\left(1-\beta_{r, n-r+1}(F(x))\right), \Phi^{-1}\left(1-\beta_{s, n-s+1}(G(y))\right)\right) .
\end{aligned}
$$

Exemplo 4.1. Considere uma amostra aleatória do vetor $(X, Y)$ que tem distribuição normal bivariada padrão com coeficiente de correlaçăo -0.5. Desejamos calcular $P\left(X_{9: 10} \leq x, Y_{10: 10} \leq y\right)$. Para $x=1.5$ e $y=1.8$, temos $F(x)=0.93319$, 
$G(y)=0.96406$ e $C(F(x), G(y))=0.897319$. O valor exato de $P\left(X_{9: 10} \leq x, Y_{10: 10} \leq\right.$ y) pode ser calculado pela equação (4.9), i.e.

$$
\begin{aligned}
P\left(X_{9: 10} \leq x, Y_{10: 10} \leq y\right) & =P\left(U_{9: 10} \leq u, V_{10: 10} \leq v\right) \\
& =P\left(U_{9: 10} \leq F(x), V_{10: 10} \leq G(y)\right) \\
& =10 \times C(F(x), G(y))^{9}[G(y)-C(F(x), G(y))]+C(F(x), G(y))^{10} \\
& =0.5902 .
\end{aligned}
$$

Para este caso temos,

$$
\rho=\frac{C(F(x), G(y))-F(x) G(y)}{\sqrt{F(x) G(y)(1-G(y))(1-F(x))}}=-0.0504337
$$

Da equação (4.12) obtém-se

$$
\begin{aligned}
H_{X_{r: n}, Y_{s: n}}(x, y) & \approx \bar{\Phi}_{\rho}\left(\bar{\Phi}^{-1}\left(\beta_{9,10-9+1}(F(x))\right), \bar{\Phi}^{-1}\left(\beta_{10,10-10+1}(G(y))\right)\right) \\
& =\bar{\Phi}_{\rho}\left(\bar{\Phi}^{-1}(0.85942), \bar{\Phi}^{-1}(0.69356)\right) \\
& =0.85942+0.69356-1+\Phi_{\rho}\left(\Phi^{-1}(1-0.85942), \Phi^{-1}(1-0.69356)\right) \\
& =0.5922 .
\end{aligned}
$$

A Tabela 4.1 apresenta uma comparação entre o valor exato obtido pela equação (4.9) com o valor aproximado obtido pela utilização da cópula assintótica dada pela equação equação (4.12). Para todos os cálculos utilizamos, $n=10, r=4, s=6$ e,

$$
\rho=\frac{C(u, v)-u v}{\sqrt{u v(1-v)(1-v)}} .
$$




\begin{tabular}{|c|c|c|c|c|c|}
\hline$C(u, v)$ & $u$ & $v$ & $\rho$ & Exato & Aprox. \\
\hline \hline $\max (u+v-1,0)$ & 0.60 & 0.40 & -1 & 0.11148 & 0.11148 \\
\hline $\max \left(1-\left[(1-u)^{1.1}+(1-v)^{1.1}\right]^{-\frac{1}{1.1}}, 0\right)$ & 0.63 & 0.38 & -0.75 & 0.0925 & 0.0938 \\
\hline$\frac{1}{10} \ln \left(1+\frac{\left(e^{10 u}-1\right)\left(e^{10 v}-1\right)}{c^{10}-1}\right)$ & 0.30 & 0.85 & -0.54 & 0.3408 & 0.3415 \\
\hline$u v+u v(1-u)(1-v)$ & 0.45 & 0.55 & -0.25 & 0.3362 & 0.3376 \\
\hline$u v$ & 0.35 & 0.70 & 0 & 0.41311 & 0.41311 \\
\hline$\frac{u v}{u+v-u v}$ & 0.42 & 0.75 & 0.25 & 0.6305 & 0.6286 \\
\hline $\exp \left(-\left((-\ln (u))^{3}+(-\ln (v))^{3}\right)^{\frac{1}{3}}\right)$ & 0.83 & 0.58 & 0.51 & 0.58221 & 0.58222 \\
\hline$\left(1+\left[\left(u^{-1}-1\right)^{5}+\left(v^{-1}-1\right)^{5}\right]^{\frac{1}{5}}\right)^{-1}$ & 0.71 & 0.58 & 0.74 & 0.58222 & 0.58218 \\
\hline $\min (u, v)$ & 0.50 & 0.50 & 1 & 0.37695 & 0.37695 \\
\hline
\end{tabular}

Tabela 4.1: Comparação entre o valor exato e aproximado de $H_{U_{4: 10}, V_{6: 10}}$.

\subsection{Relação de recorrência}

No caso univariado, existe um grande número de relações de recorrència entre as densidades e os momentos de estatísticas de ordem, veja Balakrishnan e Cohen (1991) e David (1981). Nesta seção iremos apresentar resultados similares em termos de cópulas das estatísticas de ordem.

Considere os seguinte eventos,

$$
\begin{aligned}
& A=\left\{U_{r-1: n-1} \leq u<U_{r: n-1}, V_{s: n-1} \leq v\right\}, \\
& B=\left\{U_{r-1: n-1} \leq u<U_{r: n-1}, V_{s-1: n-1} \leq v<V_{s: n-1}\right\}
\end{aligned}
$$

e

$$
D=\left\{U_{r: n} \leq u<U_{r+1: n}, V_{s: n} \leq v\right\}
$$

para $u, v \in[0,1$. Então.

$$
P(A)=P\left(U_{r-1: n-1} \leq u, V_{s: n-1} \leq v\right)-P\left(U_{r: n-1} \leq u, V_{s: n-1} \leq v\right) .
$$




$$
\begin{aligned}
P(B) & =P\left(U_{r-1: n-1} \leq u, V_{s-1: n-1} \leq v\right)-P\left(U_{r-1: n-1} \leq u, V_{s: n-1} \leq v\right) \\
& -P\left(U_{r: n-1} \leq u, V_{s-1: n-1} \leq v\right)+P\left(U_{r: n-1} \leq u, V_{s: n-1} \leq v\right)
\end{aligned}
$$

e

$$
P(D)=P\left(U_{r: n} \leq u, V_{s: n} \leq v\right)-P\left(U_{r+1: n} \leq u, V_{s: n} \leq v\right)
$$

Utilizando os resultados da Seção 4.1 obtém-se o seguinte,

$$
\begin{aligned}
P(A) & =C_{U_{r-1: n-1}, V_{s: n-1}}\left(\beta_{r-1, n-r-1}(u), \beta_{s, n-s}(v)\right)-C_{U_{r: n-1}, V_{s: n-1}}\left(\beta_{r, n-r}(u), \beta_{s, n-s}(v)\right), \\
P(B) & =C_{U_{r-1: n-1}, V_{s-1: n-1}}\left(\beta_{r-1, n-r-1}(u), \beta_{s, n-s-1}(v)\right)-C_{U_{r: n-1}, V_{s-1: n-1}}\left(\beta_{r, n-r}(u), \beta_{s, n-s-1}(v)\right) \\
& -C_{U_{r-1: n-1}, V_{s: n-1}}\left(\beta_{r-1, n-r-1}(u), \beta_{s, n-s}(v)\right)+C_{U_{r: n-1}, V_{s: n-1}}\left(\beta_{r, n-r}(u), \beta_{s, n-s}(v)\right)
\end{aligned}
$$

e

$$
P(D)=C_{U_{r: n}, V_{s: n}}\left(\beta_{r, n-r+1}(u), \beta_{s, n-s+1}(v)\right)-C_{U_{r+1: n}, V_{s: n}}\left(\beta_{r+1, n-r+2}(u), \beta_{s, n-s+1}(v)\right) .
$$

Teorema 4.2. Considerando as notações acima, a seguinte relação é válida,

$$
n P(B) C(u, v)=r P(D)+n P(A) u
$$

para $2 \leq r, s \leq n-1, n \geq 2$.

Prova. Considere uma amostra aleatória de tamanho $n$ para a qual o evento $D$ ocorre. Particionando esta amostra em duas sub-amostras. uma de tamanho $n-1$ e outra de tamanho 1 . que denotaremos de $\left(U_{l}, V_{l}\right)$. Seja

$$
E=\left\{U_{l} \leq u\right\}
$$

Então

$$
P(D \cap E)=P(E \mid D) P(D)=\frac{r}{n} P(D) .
$$


Seja $Q_{A}$ e $Q_{B}$ os eventos

$$
Q_{A}=\left\{\begin{array}{c}
\text { o evento A ocorre para a sub-amostra de tamanho } n-1 \\
\text { e } U_{l} \leq u
\end{array}\right\}
$$

e

$$
Q_{B}=\left\{\begin{array}{c}
\text { o evento B ocorre para a amostra de tamanho } n-1 \\
\text { e }\left\{U_{l} \leq u, V_{l} \leq u\right\}
\end{array}\right\}
$$

Claramente, $Q_{A}$ e $Q_{B}$ são disjuntos, e o evento $D \cap E$ ocorre se e somente se $Q_{A}$ ou $Q_{B}$ ocorre. Deste modo,

$$
\begin{aligned}
P(D \cap E) & =\frac{r P(D)}{n}=P\left(Q_{A} \cup Q_{B}\right)=P\left(Q_{A}\right)+P\left(Q_{B}\right) \\
& =u P(A)+P(U \leq u, V \leq v) P(B) \\
& =u P(A)+C(u, v) P(B) .
\end{aligned}
$$

\subsection{Fronteiras de Fréchet}

A fórmula de inclusão-exclusão afirma que se $A_{1}, \ldots, A_{n}$ são $n$ eventos, e se a probabilidade de ocorrència de no mínimo $r$ deles é denotado por $P(r ; n)$, então

$$
P(r ; n)=\sum_{m=r}^{n}(-1)^{m-r}\left(\begin{array}{c}
m-1 \\
r-1
\end{array}\right) W(m), \quad \text { onde } W(m)=\sum_{1 \leq i_{1}<\ldots<i_{m} \leq n} P\left(\cap_{j=1}^{m} A_{i_{j}}\right) .
$$

veja Feller (1968). Seja $A_{i}=\left\{U_{i} \leq u\right\}, i=1, \ldots, n$, então para a correspondente estatística de ordem $U_{r: n}$ tem-se que

$$
P\left(U_{r: n} \leq u\right)=\sum_{m=r}^{n}(-1)^{m-r}\left(\begin{array}{c}
m-1 \\
r-1
\end{array}\right) \sum_{1 \leq i_{1}<\cdots<i_{m} \leq n} P\left(\cap_{j=1}^{m}\left\{U_{i_{j}} \leq u\right\}\right) .
$$

Para encontrar a função distribuição conjunta $P\left(U_{r: n} \leq u, V_{s: n} \leq u\right)$ em termos la fórmula de inclısão-exclusão para as variáveis aleatórias dependentes $U$ e $V$, 
teremos que considerar uma extensão de (4.14) para o caso de $K \geq 2$ (finito) classes de eventos.

Seja $A_{1}, \ldots, A_{n}$ e $B_{1}, \ldots, B_{n}$ duas classes de eventos. Para inteiros $r$ e $s, 1 \leq$ $r, s \leq n$ defina

$$
P[r, s ; n]=P\left\{\text { exatamente } r A_{i} \text { 's e exatamente } s B_{i} \text { 's ocorrem }\right\}
$$

e

$$
P(r, s ; n)=P\left\{\text { no mínimo } r A_{i} \text { 's e no mínimo } s B_{i} \text { 's ocorrem }\right\} \text {. }
$$

Seja

$$
W(r, s)=\sum P\left(\cap_{j=1}^{r} A_{i_{j}} \cap_{i=1}^{s} B_{j_{i}}\right)
$$

em que $\sum$ denota a somatória sobre os índices $1 \leq i_{1}<\cdots<i_{r} \leq n ; 1 \leq j_{1}<$ $\cdots<i_{s} \leq n$. Em Meyer (1969) encontramos os seguintes resultados,

$$
P[r, s ; n]=\sum_{t=r+s}^{2 n} \sum_{i+j=t}(-1)^{t-r-s}\left(\begin{array}{l}
i \\
r
\end{array}\right)\left(\begin{array}{l}
j \\
s
\end{array}\right) W(i, j)
$$

e,

$$
P(r, s ; n)=\sum_{\alpha=r}^{n} \sum_{\beta=s}^{n} P[\alpha, \beta ; n]=\sum_{i=r}^{n} \sum_{j=s}^{n}(-1)^{i+j-(r+s)}\left(\begin{array}{l}
i-1 \\
r-1
\end{array}\right)\left(\begin{array}{l}
j-1 \\
s-1
\end{array}\right) W(i, j) .
$$

Deste modo, a forma bivariada das desigualdades de Bonferroni para qualquer inteiro não negativo $k \geq 0$ é dado por

$$
\sum_{t=r+s}^{r+s+2 k+1} \sum_{i+j=t} g(i, j, t) \leq P(r, s ; n) \leq \sum_{t=r+s}^{r+s+2 k} \sum_{i+j=t} g(i, j, t)
$$

em que

$$
g(i, j, t)=(-1)^{t-(r+s)}\left(\begin{array}{l}
i-1 \\
r-1
\end{array}\right)\left(\begin{array}{l}
j-1 \\
s-1
\end{array}\right) W(i, j),
$$

veja Meyer (1969). Para $2 k \geq n-r-s$ as fronteiras coincidem.

O objetivo é determinar fronteiras para $C_{U_{r: n} . V_{s: n}}$ utilizando $(4.15)$ e para tanto precisamos especificar a função $W(i, j)$. 
Considere os eventos

$$
A_{i}=\left\{U_{i} \leq \beta_{r, n-r+1}^{-1}(w)\right\} \quad \text { e } \quad B_{i}=\left\{V_{i} \leq \beta_{s, n-s+1}^{-1}(t)\right\}
$$

para $U_{i}, V_{i} \sim U(0,1), i=1, \ldots, n$, distribuídos numa matriz $2 \times n$. Entào,

$$
P(r, s ; n)=P\left(U_{r: n} \leq \beta_{r, n-r+1}^{-1}(w), V_{s: n} \leq \beta_{s, n-s+1}^{-1}(t)\right)=C_{U_{r: n}, V_{s: n}}(w, t) .
$$

Os elementos de diferentes colunas são independentes e cada elemento em qualquer coluna tem a mesma probabilidade. Então a contribuição para $W(i, j)=$ $\sum_{d} T(i, j ; d)$, deste elementos consiste de $d$ pares $A_{i} \cap B_{i}$ e os restantes são tomados de diferentes colunas, i.e.,

$$
\begin{aligned}
T(i, j ; d) & =\left(\begin{array}{l}
n \\
d
\end{array}\right)\left[C\left(\beta_{r, n-r+1}^{-1}(w), \beta_{s, n-s+1}^{-1}(t)\right)\right]^{d} \\
& \times\left(\begin{array}{c}
n-d \\
i-d
\end{array}\right)\left[\beta_{r, n-r+1}^{-1}(w)\right]^{i-d} \times\left(\begin{array}{c}
n-i \\
j-d
\end{array}\right)\left[\beta_{s, n-s+1}^{-1}(t)\right]^{j-d},
\end{aligned}
$$

veja Galambos (1975). A equação (4.15) pode parecer complicada, entretanto seu cálculo não é difícil. por exemplo, para $k=0$ em (4.15) obtém-se as seguintes fronteiras,

$$
W(r, s)-r W(r+1, s)-s W(r, s+1) \leq C_{U_{r: n}, V_{s: n}}(w, t) \leq W(r, s) .
$$

Para ilustrar a metodologia, damos dois exemplos numéricos.

Exemplo 4.2. Para os dados do exemplo 4.1 calculamos $w=0.8594, t=0.6936$ e o valor exato $C_{C_{9: 10}, V_{10: 10}}(w, t)=0.5902$. Deste modo, encontramos as seguintes fronteiras:

$$
\begin{array}{ll}
k=0: & 0.5902 \leq C_{U_{9: 10}, V_{10: 10}}(w, t) \leq 3.6361 \\
k=1: & 0.5902 \leq C_{U_{9: 10}, V_{10: 10}}(w, t) \leq 0.5902
\end{array}
$$

e nenhuma correçào pode ser obtida pelo aumento de $k$ em (4.15). 
Exemplo 4.3. Consideremos os seguintes valores: $n, r=2, s=3 w=0.1405798$ e $t=0.9980501$. O valor exato de $C_{U_{2: 10}, V_{3: 10}}(w, t)$ é 0.1398483 e as fronteiras de Fréchet são os seguintes:

$$
\begin{array}{ll}
k=3: & 0.1255057 \leq C_{U_{2: 10}, V_{3: 10}}(w, t) \leq 0.3034248 \\
k=4: & 0.1398175 \leq C_{U_{2: 10}, V_{3: 10}}(w, t) \leq 0.1406579 ; \\
k=5: & 0.1398482 \leq C_{U_{2: 10}, V_{3: 10}}(w, t) \leq 0.1398491 ; \\
k=6: & 0.1398483 \leq C_{U_{2: 10}, V_{3: 10}}(w, t) \leq 0.1398483
\end{array}
$$

e nenhuma correção pode ser obtida pelo aumento de $k$ em (4.15).

Neste capítulo apresentamos alguns resultados relacionados com a estrutura de dependència entre estatísticas de ordem de vetores bivariados $(X, Y)$ que resultaram nos seguintes artigos Anjos et al. (2004b) e Kolev et al. (2005). 


\section{Capítulo 5}

\section{Cópulas com marginais multivariadas}

A teoria de cópulas é fundamentada no Teorema de Sklar e nos fornece ferramentas para construirmos famílias de distribuições multivariadas a partir de um conjunto de funções distribuição univariadas conhecidas. A teoria de cópulas portanto, nos fornece várias ferramentas para que possamos estudar a estrutura de dependència entre variáveis aleatórias. Entretanto, se desejarmos estudar a estrutura de dependència entre vetores aleatórios, não encontramos nenhuma ferramenta tão geral e efetiva quanto a teoria de cópulas.

Quando as marginais são multivariadas, i.e., quando temos vetores aleatórios, as pesquisas tèm sido direcionadas principalmente para o estudo da compatibilidade entre as distribuições multivariadas de vetores que possuem variáveis em comum, veja Joe (1997, Capítulo 3). Por exemplo para $n=3$. considere a classe $\mathcal{H}\left(H_{X_{1} X_{2}}, H_{X_{1} X_{3}}\right)$ com marginais bivariadas fixas ou conhecidas $H_{X_{1} X_{2}}$ e $H_{X_{1} X_{3}}$, neste caso, assumimos que a variável em comım é $X_{1}$, portanto $H_{X_{1} X_{2}}(., \infty)=$ $H_{X_{1} X_{3}}(., \infty)=F_{X_{1}}($.$) . Esta classe é sempre não vazia pois ela contém distribuiçòes$ trivariadas tais que $X_{2}$ e $X_{3}$ sào condicionalmente independentes dado $X_{1}$, i.e.. sem- 
pre é possível encontrar a distribuição conjunta

$$
H\left(x_{1}, x_{2}, x_{3}\right)=\int_{-\infty}^{x_{1}} H_{X_{2} \mid X_{1}}\left(x_{2} \mid x\right) H_{X_{3} \mid X_{1}}\left(x_{3} \mid x\right) d F_{X_{1}}(x) .
$$

Além disso, note que em tais casos os limitantes de Fréchet,

$$
\min \left\{F_{X_{1}}\left(x_{1}\right), F_{X_{2}}\left(x_{2}\right), F_{X_{3}}\left(x_{3}\right)\right\} \quad \text { e } \max \left\{F_{X_{1}}\left(x_{1}\right)+F_{X_{2}}\left(x_{2}\right)+F_{X_{3}}\left(x_{3}\right)-2,0\right\}
$$

podem ser melhorados, veja Joe (1997). A relação (5.1) pode ser estendida para uma distribuição $n$-variada, dada duas $(n-1)$-dimensional marginais, contendo $(n-2)$ variáveis em comum.

Se considerarmos a classe $\mathcal{H}\left(H_{X_{1} X_{2}}, H_{X_{1} X_{3}}, H_{X_{2} X_{3}}\right)$ com marginais bivariadas fixas ou conhecidas $H_{X_{1} X_{2}}, H_{X_{2} X_{3}}$ e $H_{X_{2} X_{3}}$, então as condições de compatibilidade são obtidas considerando duas das trés marginais arbitrárias e a terceira marginal bivariada tendo restrições dadas pelas outra duas marginais. As condições de unicidade e compatibilidade são discutidas em Joe (1997), veja também Dall'Aglio (1972) para algumas condições necessárias.

Portanto, não pode-se simplesmente selecionar uma família paramétrica de funções e esperar que elas gerem uma nova distribuição multidimensional válida. De um modo geral, uma família de distribuições multivariadas pode ser construída utilizando métodos tais como misturas, representações estocásticas, etc, veja Joe (1997, Capítulo 4).

A teoria de cópulas, entretanto, é devotada ao estudo de classes de distribuiçòes $n$-variadas $\mathcal{H}\left(F_{X_{1}}, \ldots, F_{X_{n}}\right)$, em que as marginais fixas ou conhecidas são univariadas $F_{X_{1}}, \ldots, F_{X_{n}}$. Portanto, as cópulas são uma das ferramentas mais íteis para lidar com distribuições multivariadas com marginais unidimensionais fixas ou conhecidas. 
A insuficiência das cópulas para lidar com distribuições multivariadas cujas marginais fixas ou conhecidas são multivariadas é ilustrada no paradoxo da cópula de Nutshell discutido em Genest et al. (1995). Eles mostram que a única possibilidade para que,

$$
H\left(x_{1}, \ldots, x_{n_{1}}, x_{n_{1}+1}, \ldots, x_{n_{1}+n_{2}}\right)=C\left(H_{n_{1}}\left(x_{1}, \ldots, x_{n_{1}}\right), H_{n_{2}}\left(x_{n_{1}+1}, \ldots, x_{n_{1}+n_{2}}\right)\right)
$$

defina uma distribuição $\left(n_{1}+n_{2}\right)$-dimensional válida, $n_{1}+n_{2} \geq 3$, para todo $H_{n_{1}}$ e $H_{n_{2}}$ (com dimensões $n_{1}$ e $n_{2}$, respectivamente) é $C(u, v)=u v$, i.e. cópula da independência.

As dificuldades de se construir uma distribuição marginal com marginais multivariadas fixas ou conhecidas são bem conhecidas. Alguns aspectos deste problema são discutidos em Dall'Aglio (1972). O problema da existència foi resolvido em Kellerer (1964a) mas a solução é essencialmente teórica e não permite o uso dos resultados na maioria dos casos práticos. Os casos que permitem uma construção simples são classificados em Kellerer (1964b). Um exemplo típico é quando uma distribuição pode ser construída como uma distribuição de uma cadeia de Markov.

Marco e Ruiz-Rivas (1992) estudam o seguinte problema: dado $d$ possíveis distribuições marginais associadas a vetores que não possuem termos em comum $H_{1}, \ldots, H_{d}$ de dimensões $n_{1}, \ldots, n_{d}$, respectivamente, quais as condições para que uma função $d$-dimensional $C_{d}$ deve satisfazer para que $C_{d}\left(H_{1}, \ldots, H_{d}\right)$ seja uma função distribuição $\left(\sum_{i=1}^{d} n_{i}\right)$-dimensional. Neste mesmo artigo encontramos $1 \mathrm{~m}$ procedimento para se construir tal função.

Cuadras (1992) fornece ım método para construir distribuições multivariadas em que ambas as marginais multivariadas e a matriz de correlação são dadas. Este método prodız uma família de distribuições que são totalmente regressivas lineares e 
podem ser úteis para gerar modelos para testes estatísticos, e.g. modelos estruturais em que a estrutura de covariància é dada mas a distribuição de probabilidade é diferente da normal multivariada.

Li et al. (1996) introduziram uma nova ferramenta, chamada "linkage" (baseado em distribuições condicionais), que é útil para lidar com distribuições multivariadas cujas marginais especificadas não são univariadas. Esta ferramenta é particularmente interessante quando nem todas as relações entre as variáveis são igualmente importantes, mas somente as relações entre os vetores aleatórios são relevantes. Em Li et al. (1999) os autores fazem uma extensão da função "linkage" para "linkage" dinâmica, que pode ser usada para modelar distribuições multivariadas de variáveis aleatórias não negativas, com a vantagem de levar em conta a dinâmica temporal dos tempos de vida associados, i.e. esta modelagem leva em conta a ordem temporal em que os eventos acorrem.

Cohen (1984) sugere um procedimento que fornece distribuições conjuntas para dadas marginais multivariadas especificadas para o caso em que os vetores aleatórios associados a estas distribuição não possuem termos em comum. Na Seção 5.1 adaptamos o método de Cohen para cópulas.

Na Seção 5.2 apresentamos uma nova ferramenta que é completamente diferente das metodologias sugeridas até o momento. A proposta é fornecer um método simples, baseado na distribuição de Kendall veja Nelsen et al. (2003), a fim de modelar a estrutura de dependència entre $d$ vetores aleatórios $\left(X_{1}, \ldots, X_{m_{1}}\right), \ldots,\left(X_{m_{d-1}+1}, \ldots\right.$, $\left.X_{m_{d}}\right)$, com $m_{d}=n$ utilizando uma cópula $d$-dimensional $(2 \leq d \leq n)$ 


\subsection{O método de Cohen para cópulas}

Cohen (1984) apresenta um método simples para obter uma densidade conjunta $h$ do vetor aleatório $\left(X_{1}, \ldots, X_{n_{1}+n_{2}}\right)$ a qual tem densidades marginais fixas ou conhecidas $h_{1}$ de $\left(X_{1}, \ldots, X_{n_{1}}\right)$ e $h_{2}$ de $\left(X_{n_{1}+1}, \ldots, X_{n_{1}+n_{2}}\right)$. Considera-se apenas os casos em que os vetores não tèm variáveis em comum.

Apresentamos a seguir o método de Cohen adaptado para cópulas. Suponha que conhecemos as cópulas $C_{1}$ e $C_{2}$ associados aos vetores aleatórios $\left(X_{1}, \ldots, X_{n_{1}}\right)$ e $\left(X_{n_{1}+1}, \ldots, X_{n_{1}+n_{2}}\right)$, respectivamente. Deseja-se obter a cópula $C$ associada ao vetor $\left(X_{1}, \ldots, X_{n_{1}+n_{2}}\right)$ consistente com as marginais multivariadas dadas, i.e.,

$$
C_{1}\left(u_{1}, \ldots, u_{n_{1}}\right)=C\left(u_{1}, \ldots, u_{n_{1}}, 1, \ldots, 1\right)
$$

e

$$
C_{2}\left(u_{n_{1}+1}, \ldots, u_{n_{1}+n_{2}}\right)=C\left(1, \ldots 1, u_{n_{1}+1}, \ldots, u_{n_{1}+n_{2}}\right)
$$

Para tanto. escolhe-se uma função $C_{D}$ que pode ser uma cópula absolutamente contínua ou qualquer outra função que satisfaça

$$
\int_{0}^{1} \cdots \int_{0}^{1} c_{D}\left(u_{1}, \ldots, u_{n_{1}+n_{2}}\right)=1
$$

em que

$$
c_{D}=\frac{\partial^{n_{1}+n_{2}} C_{D}\left(u_{1}, \ldots u_{n_{1}+n_{2}}\right)}{\partial u_{1} \cdots \partial u_{n_{1}+n_{2}}}
$$

fornecerá a estrutura de dependència entre os vetores $\left(X_{1} \ldots \ldots X_{n_{1}}\right)$ e $\left(X_{n_{1}+1} \ldots \ldots X_{n_{1}+n_{2}}\right)$.

Um número infinito de estruturas de dependència satisfazendo as condiçoes (5.2) 
e (5.3) podem ser gerado da seguinte forma:

$$
\begin{aligned}
C\left(u_{1}, \ldots, u_{n_{1}+n_{2}}\right) & =C_{1}\left(u_{1}, \ldots, u_{n_{1}}\right) C_{2}\left(u_{n_{1}+1}, \ldots, u_{n_{1}+n_{2}}\right) \\
& +a \int_{0}^{u_{1}} \ldots \int_{0}^{u_{n_{1}+n_{2}}} c_{1}\left(v_{1}, \ldots, v_{n_{1}}\right) c_{2}\left(v_{n_{1}+1}, \ldots, v_{n_{1}+n_{2}}\right) \\
& \times \delta\left(w_{1}, \ldots, w_{n_{1}+n_{2}}\right) d v_{1} \cdots d v_{n_{1}+n_{2}} .
\end{aligned}
$$

em que

$$
c_{1}\left(u_{1}, \ldots, u_{n_{1}}\right)=\frac{\partial^{n_{1}} C_{1}\left(u_{1}, \ldots, u_{n_{1}}\right)}{\partial u_{1} \cdots \partial u_{n_{1}}}
$$

e

$$
c_{2}\left(u_{n_{1}+1}, \ldots, u_{n_{1}+n_{2}}\right)=\frac{\partial^{n_{2}} C_{2}\left(u_{n_{1}+1}, \ldots, u_{n_{1}+n_{2}}\right)}{\partial u_{n_{1}+1} \cdots \partial u_{n_{1}+n_{2}}}
$$

são as densidades das cópulas $C_{1}$ e $C_{2}$ respectivamente,

$$
\begin{aligned}
& w_{i}=w_{i}\left(u_{1}, \ldots, u_{n_{1}}\right), \quad i=1, \ldots, n_{1} \\
& w_{i}=w_{i}\left(u_{n_{1}+1}, \ldots, u_{n_{1}+n_{2}}\right), \quad i=n_{1}+1, \ldots, n_{1}+n_{2}
\end{aligned}
$$

e

$$
\begin{aligned}
\delta\left(w_{1}, \ldots, w_{n_{1}+n_{2}}\right) & =c_{D}\left(w_{1}, \ldots, w_{n_{1}+n_{2}}\right)-c_{D}\left(w_{1}, \ldots, w_{n_{1}}\right) \\
& -c_{D}\left(w_{n_{1}+1}, \ldots, w_{n_{1}+n_{2}}\right)+1 .
\end{aligned}
$$

Na relação (5.4) os argumentos $w_{i}$ 's são determinados em termos de $u_{i}$ 's de tal forma que o jacobiano da transformação satisfaça $\mathbf{J}_{1}=c_{1}\left(u_{1}, \ldots, u_{n_{1}}\right)$ para $i=1, \ldots, n_{1} \mathrm{e}$ $\mathrm{J}_{2}=c_{2}\left(u_{n_{1}+1}, \ldots, u_{n_{1}+n_{2}}\right)$ para $i=n_{1}+1, \ldots, n_{1}+n_{2}$. Uma possível transformação que satisfaz estas condições é a seguinte:

$$
w_{i}=\frac{\frac{\partial^{n_{1}-i} C_{1}\left(u_{i}, \ldots, u_{n_{1}}\right)}{\partial u_{i+1} \cdots \partial u_{n_{1}}}}{\frac{\partial^{n_{1}-i} C_{1}\left(u_{i+1}, \ldots, u_{n_{1}}\right)}{\partial u_{i+1} \cdots \partial u_{n_{1}}}}=\frac{\frac{\partial^{n_{1}-i} C_{1}\left(u_{i}, \ldots, u_{n_{1}}\right)}{\partial u_{i+1} \cdots \partial u_{n_{1}}}}{c_{1}\left(u_{i+1}, \ldots, u_{n_{1}}\right)}, \quad i=1, \ldots, n_{1}-2
$$




$$
w_{i}=\frac{\frac{\partial^{n_{2}-i} C_{2}\left(u_{i}, \ldots, u_{n_{1}+n_{2}}\right)}{\partial u_{i+1} \cdots \partial u_{n_{1}+n_{2}}}}{\frac{\partial^{n_{2}-i} C_{2}\left(u_{i+1}, \ldots, u_{n_{1}+n_{2}}\right)}{\partial u_{i+1} \cdots \partial u_{n_{1}+n_{2}}}}=\frac{\frac{\partial^{n_{2}-i} C_{2}\left(u_{i}, \ldots, u_{n_{1}+n_{2}}\right)}{\partial u_{i+1} \cdots \partial u_{n_{1}+n_{2}}}}{c_{2}\left(u_{i+1}, \ldots, u_{n_{1}+n_{2}}\right)}, \quad i=n_{1}+1, \ldots, n_{1}+n_{2}-2
$$

e

$$
w_{i}= \begin{cases}\frac{\partial C\left(u_{n_{1}-1}, u_{n_{1}}\right)}{\partial u_{n_{1}}} & \text { se } i=n_{1}-1 \\ u_{n_{1}} & \text { se } i=n_{1} \\ \frac{\partial C\left(u_{n_{1}+n_{2}-1}, u_{n_{1}+n_{2}}\right)}{\partial u_{n_{1}+n_{2}}} & \text { se } i=n_{1}+n_{2}-1 \\ u_{n_{1}+n_{2}} & \text { se } i=n_{1}+n_{2}\end{cases}
$$

Nestas condições, segue que

$$
\frac{\partial w_{i}}{\partial u_{j}}=0 \quad \text { para } \quad j<i
$$

portanto o jacobiano da transformação será o produto dos elementos da diagonal. i.e..

$$
\mathbf{J}_{\mathbf{1}}=\frac{\partial w_{1}}{\partial u_{1}} \times \frac{\partial w_{2}}{\partial u_{2}} \times \cdots \times \frac{\partial w_{n_{1}}}{\partial u_{n_{1}}}
$$

e

$$
\mathrm{J}_{2}=\frac{\partial w_{n_{1}+1}}{\partial u_{n_{1}+1}} \times \frac{\partial w_{n_{1}+2}}{\partial u_{n_{1}+2}} \times \cdots \times \frac{\partial w_{n_{1}+n_{2}}}{\partial u_{n_{1}+n_{2}}} .
$$

Das relações (5.5), (5.6) e (5.7) obtém-se,

$$
\frac{\partial w_{i}}{\partial u_{i}}= \begin{cases}\frac{c_{1}\left(u_{i}, \ldots, u_{n_{1}}\right)}{c_{1}\left(u_{i+1}, \ldots, u_{n_{1}}\right)} & \text { se } i=1, \ldots, n_{1}-2 \\ c\left(u_{n_{1}-1}, u_{n_{1}}\right) & \text { se } i=n_{1}-1 \\ 1 & \text { se } i=n_{1}\end{cases}
$$

e

$$
\frac{\partial w_{i}}{\partial u_{i}}= \begin{cases}\frac{c_{2}\left(u_{i}, \ldots, u_{n_{1}+n_{2}}\right)}{c_{2}\left(u_{i+1} \ldots, u_{n_{1}+n_{2}}\right)} & \text { se } i=n_{1}+1, \ldots, n_{1}+n_{2}-2 \\ c\left(u_{n_{1}+n_{2}-1}, u_{n_{1}+n_{2}}\right) & \text { se } i=n_{2}-1 \\ 1 & \text { se } i=n_{2}\end{cases}
$$

Substituindo as relações (5.10) e (5.11) em (5.8) e (5.9), segue que

$$
\begin{aligned}
J_{1} & =\frac{c_{1}\left(u_{1}, \ldots, u_{n_{1}}\right)}{c_{1}\left(u_{2}, \ldots, u_{n_{1}}\right)} \times \frac{c_{1}\left(u_{2}, \ldots, u_{n_{1}}\right)}{c_{1}\left(u_{3}, \ldots, u_{n_{1}}\right)} \times \cdots \\
& \times \frac{c_{1}\left(u_{n_{1}-2}, u_{n_{1}-1}, u_{n_{1}}\right)}{c_{1}\left(u_{n_{1}-1}, u_{n_{1}}\right)} \times c_{1}\left(u_{n_{1}-1}, u_{n_{1}}\right) \\
& =c_{1}\left(u_{1}, \ldots, u_{n_{1}}\right)
\end{aligned}
$$


e

$$
\begin{aligned}
\mathbf{J}_{2} & =\frac{c_{2}\left(u_{n_{1}+1}, \ldots, u_{n_{1}+n_{2}}\right)}{c_{2}\left(u_{n_{1}+2}, \ldots, u_{n_{1}+n_{2}}\right)} \times \frac{c_{2}\left(u_{n_{1}+2}, \ldots, u_{n_{1}+n_{2}}\right)}{c_{2}\left(u_{n_{1}+3}, \ldots, u_{n_{1}+n_{2}}\right)} \times \ldots \\
& \times \frac{c_{2}\left(u_{n_{1}+n_{2}-2}, u_{n_{1}+n_{2}-1}, u_{n_{1}+n_{2}}\right)}{c_{2}\left(u_{n_{1}+n_{2}-1}, u_{n_{1}+n_{2}}\right)} \times c_{2}\left(u_{n_{1}+n_{2}-1}, u_{n_{1}+n_{2}}\right) \\
& =c_{2}\left(u_{n_{1}+1}, \ldots, u_{n_{1}+n_{2}}\right)
\end{aligned}
$$

A partir dessas condições, verifica-se que,

$$
\begin{aligned}
& c\left(u_{1}, \ldots, u_{n_{1}}\right)=\int_{0}^{1} \ldots \int_{0}^{1} c_{1}\left(u_{1}, \ldots, u_{n_{1}}\right) c_{2}\left(u_{n_{1}+1}, \ldots, u_{n_{1}+n_{2}}\right) \\
& \times\left[1+a \delta\left(w_{1}, \ldots, w_{n_{1}+n_{2}}\right)\right] d u_{n_{1}+1} \cdots d u_{n_{1}+n_{2}} \\
& =c_{1}\left(u_{1}, \ldots, u_{n_{1}}\right)[\underbrace{\int_{0}^{1} \ldots \int_{0}^{1} c_{2}\left(u_{n_{1}+1}, \ldots, u_{n_{1}+n_{2}}\right) d u_{n_{1}+1} \cdots d u_{n_{1}+n_{2}}}_{=1} \\
& \left.+a \int_{0}^{1} \ldots \int_{0}^{1} c_{2}\left(u_{n_{1}+1}, \ldots, u_{n_{1}+n_{2}}\right) \delta\left(w_{1}, \ldots, w_{n_{1}+n_{2}}\right) d u_{n_{1}+1} \cdots d u_{n_{1}+n_{2}}\right] \\
& c\left(u_{1}, \ldots, u_{n_{1}}\right)=c_{1}\left(u_{1}, \ldots, u_{n_{1}}\right)[1+ \\
& +a \underbrace{\int_{0}^{1} \ldots \int_{0}^{1} c_{2}\left(u_{n_{1}+1}, \ldots, u_{n_{1}+n_{2}}\right) c_{D}\left(w_{1}, \ldots, w_{n_{1}+n_{2}}\right) d u_{n_{1}+1} \cdots d u_{n_{1}+n_{2}}}_{A_{1}} \\
& -a c_{D}\left(w_{1}, \ldots, w_{n_{1}}\right) \underbrace{\int_{0}^{1} \cdots \int_{0}^{1} c_{2}\left(u_{n_{1}+1}, \ldots, u_{n_{1}+n_{2}}\right) d u_{n_{1}+1} \cdots d u_{n_{1}+n_{2}}}_{=1} \\
& -a \underbrace{\int_{0}^{1} \ldots \int_{0}^{1} c_{2}\left(u_{n_{1}+1}, \ldots, u_{n_{1}+n_{2}}\right) c_{D}\left(w_{n_{1}+1}, \ldots, w_{n_{1}+n_{2}}\right) d u_{n_{1}+1} \cdots d u_{n_{1}+n_{2}}}_{A_{2}} \\
& +a \underbrace{\int_{0}^{1} \cdots \int_{0}^{1} c_{2}\left(u_{n_{1}+1}, \ldots, u_{n_{1}+n_{2}}\right) d u_{n_{1}+1} \cdots d u_{n_{1}+n_{2}}}_{=1}]
\end{aligned}
$$


Das relações (5.9) e (5.13) tem-se para a integral $A_{1}$ o seguinte,

$$
\begin{aligned}
A_{1} & =\int_{0}^{1} \ldots \int_{0}^{1} c_{D}\left(w_{1}, \ldots, w_{n_{1}+n_{2}}\right) \frac{\partial w_{n_{1}+1}}{\partial u_{n_{1}+1}} \times \frac{\partial w_{n_{1}+2}}{\partial u_{n_{1}+2}} \times \cdots \times \frac{\partial w_{n_{1}+n_{2}}}{\partial u_{n_{1}+n_{2}}} d u_{n_{1}+1} \cdots d u_{n_{1}+n_{2}} \\
& =\int_{0}^{1} \ldots \int_{0}^{1} c_{D}\left(w_{1}, \ldots, w_{n_{1}+n_{2}}\right) d w_{n_{1}+1} \cdots d w_{n_{1}+n_{2}} \\
& =c_{D}\left(w_{1}, \ldots, w_{n_{1}}\right)
\end{aligned}
$$

e para $A_{2}$,

$$
\begin{aligned}
A_{2} & =\int_{0}^{1} \cdots \int_{0}^{1} c_{D}\left(w_{n_{1}+1}, \ldots, w_{n_{1}+n_{2}}\right) \frac{\partial w_{n_{1}+1}}{\partial u_{n_{1}+1}} \times \frac{\partial w_{n_{1}+2}}{\partial u_{n_{1}+2}} \times \cdots \times \frac{\partial w_{n_{1}+n_{2}}}{\partial u_{n_{1}+n_{2}}} d u_{n_{1}+1} \cdots d u_{n_{1}+n_{2}} \\
& =\int_{0}^{1} \cdots \int_{0}^{1} c_{D}\left(w_{n_{1}+1}, \ldots, w_{n_{1}+n_{2}}\right) d w_{n_{1}+1} \cdots d w_{n_{1}+n_{2}} \\
& =1 .
\end{aligned}
$$

A partir destes fatos, segue que $C\left(u_{1}, \ldots, u_{n_{1}}\right)=C_{1}\left(u_{1}, \ldots, u_{n_{1}}\right)$. Similarmente, das relações $(5.8)$ e $(5.12)$ verifica-se também que $C\left(u_{n_{1}+1}, \ldots, u_{n_{1}+n_{2}}\right)=C_{2}\left(u_{n_{1}+1}, \ldots, u_{n_{1}+n_{2}}\right)$. $\mathrm{O}$ que prova que a cópula $C$ é consistente com as marginais especificadas $C_{1}$ e $C_{2}$. A constante $a$ é tal que $c\left(u_{1}, \ldots, u_{n_{1}+n_{2}}\right) \geq 0$ para todo $u_{i} \in[0,1], i=1, \ldots, n_{1}+n_{2}$, e portanto $a \in\left[-a_{2}^{-1}, a_{1}^{-1}\right]$, onde $-a_{1}$ e $a_{2}$ são o mínimo e o máximo absolutos de $\delta\left(w_{1}, \ldots, w_{n_{1}+n_{2}}\right)$.

Exemplo 5.1. Seja $C_{1}\left(u_{1}, u_{2}\right)$ e $C_{2}\left(u_{3}, u_{4}\right)$ as cópulas associadas aos pares $\left(U_{1}, U_{2}\right)$ e $\left(U_{3}, U_{4}\right)$, respectivamente. Seja $c_{D}\left(w_{1}, w_{2}, w_{3}, w_{4}\right)=2^{4} w_{1} w_{2} w_{3} w_{4}$ com $n_{1}=n_{2}=2$. E fácil ver que

$$
\int_{0}^{1} \int_{0}^{1} \int_{0}^{1} \int_{0}^{1} c_{D}\left(w_{1}, w_{2}, w_{3}, w_{4}\right) d w_{1} d w_{2} d w_{3} d w_{4}=1
$$

$\mathrm{com}$

$$
c_{D}\left(w_{1}, w_{2}\right)=\int_{0}^{1} \int_{0}^{1} 2^{4} w_{1} w_{2} w_{3} w_{4} d w_{3} d w_{4}=4 w_{1} w_{2}
$$

e

$$
c_{D}\left(w_{3}, w_{4}\right)=\int_{0}^{1} \int_{0}^{1} 2^{4} w_{1} w_{2} w_{3} w_{4} d w_{1} d w_{2}=4 w_{3} u_{4} .
$$


Das relações (5.5) e (5.6) segue que

$$
w_{1}=\frac{\partial C_{1}\left(u_{1}, u_{2}\right)}{\partial u_{2}}, \quad w_{2}=u_{2}, \quad w_{3}=\frac{\partial C_{2}\left(u_{3}, u_{4}\right)}{\partial u_{4}} \quad \text { e } \quad w_{4}=u_{4} .
$$

Assim,

$$
\begin{aligned}
\delta\left(w_{1}, w_{2}, w_{3}, w_{4}\right) & =c_{D}\left(w_{1}, w_{2}, w_{3}, w_{4}\right)-c_{D}\left(w_{1}, w_{2}\right)-c_{D}\left(w_{3}, w_{4}\right)+1 \\
& =\left[c_{D}\left(w_{1}, w_{2}\right)-1\right]\left[c_{D}\left(w_{3}, w_{4}\right)-1\right] \\
& =\left[4 w_{1} w_{2}-1\right]\left[4 w_{3} w_{4}-1\right] .
\end{aligned}
$$

Então a densidade de cópula c associada ao vetor $\left(U_{1}, U_{2}, U_{3}, U_{4}\right)$ é dada por

$$
\begin{aligned}
c\left(u_{1}, u_{2}, u_{3}, u_{4}\right) & =c_{1}\left(u_{1}, u_{2}\right) c_{2}\left(u_{3}, u_{4}\right) \\
& \times\left\{1+a\left[4 u_{2} \frac{\partial C_{1}\left(u_{1}, u_{2}\right)}{\partial u_{2}}-1\right]\left[4 u_{4} \frac{\partial C_{2}\left(u_{3}, u_{4}\right)}{\partial u_{4}}-1\right]\right\} .
\end{aligned}
$$

A constante a dependerá da transformação escolhida da função $C_{D}$ e das cópulas $C_{1} e C_{2}$.

Exemplo 5.2. Seja $C_{D}$ a cópula da independência, i.e., $C_{D}\left(w_{1}, w_{2}, w_{3}, w_{4}\right)=w_{1} w_{2} w_{3} w_{4}$, então $\delta\left(w_{1}, w_{2}, w_{3}, w_{4}\right)=0$. Considerando as mesmas funçóes $w_{1}, w_{2}, w_{3}$ e $w_{4}$ do exemplo anterior, segue que

$$
C\left(u_{1}, u_{2}, u_{3}, u_{4}\right)=C_{1}\left(u_{1}, u_{2}\right) C_{2}\left(u_{3}, u_{4}\right) .
$$

Note que neste caso a cópula $C_{D}$ fornece exatamente a estrutura de dependência entre os vetores $\left(U_{1}, U_{2}\right)$ e $\left(U_{3}, U_{4}\right)$, o que no caso geral nào é verificado.

Utilizando a versão multivariada do coeficiente de Kendall, veja Nelsen (2002). tem-se para o caso em que os vetores $\left(X_{1}, \ldots, X_{n_{1}}\right)$ e $\left(X_{n_{1}+1}, \ldots, X_{n_{1}+n_{2}}\right)$ são inde- 
pendentes, i.e., $\delta\left(w_{1}, w_{2}, w_{3}, w_{4}\right)=0$ o seguinte:

$$
\begin{aligned}
\tau_{S} & =\frac{1}{2^{n_{1}+n_{2}-1}-1}\left[2^{n_{1}+n_{2}} \int_{0}^{1} \cdots \int_{0}^{1} C\left(u_{1}, \ldots, u_{n_{1}+n_{2}}\right) d C\left(u_{1}, \ldots, u_{n_{1}+n_{2}}\right)-1\right] \\
& =\frac{1}{2^{n_{1}+n_{2}-1}-1}\left[2^{n_{1}+n_{2}} \int_{0}^{1} \cdots \int_{0}^{1} C_{1}\left(u_{1}, \ldots, u_{n_{1}}\right) d C_{1}\left(u_{1}, \ldots, u_{n_{1}}\right)\right. \\
& \left.\times \int_{0}^{1} \ldots \int_{0}^{1} C_{2}\left(u_{n_{1}+1}, \ldots, u_{n_{1}+n_{2}}\right) C_{2}\left(u_{n_{1}+1}, \ldots, u_{n_{1}+n_{2}}\right)-1\right]
\end{aligned}
$$

Portanto se os vetores $\left(X_{1}, \ldots, X_{n_{1}}\right)$ e $\left(X_{n_{1}+1}, \ldots, X_{n_{1}+n_{2}}\right)$ forem independentes não implicará $\tau_{S}=0$.

\subsection{Cópulas para vetores aleatórios não sobrepostos}

Considere o seguinte conjunto de variáveis aleatórias $X_{1}, \ldots, X_{n}$ com funções distribuição marginais univariadas contínuas $F_{X_{1}}\left(x_{1}\right), \ldots, F_{X_{n}}\left(x_{n}\right)$. Supondo que estão separadas em $d$ aglomerados $2 \leq d \leq n$, e seja o $l$-ésimo aglomerado contendo $n_{l}$ variáveis $\bar{X}_{l}=\left(X_{m_{l-1}+1}, \ldots, X_{m_{l}}\right)$ com $m_{l}=\sum_{j=1}^{l} n_{j}, m_{0}=0, l=1, \ldots, d$, e $n=n_{1}+\cdots+n_{d}$. Supomos que os aglomerados podem ser inclusive independentes. Assumindo que conhecemos a função distribuição conjunta de cada aglomerado

$$
H_{l}\left(x_{m_{l-1}+1}, \ldots, x_{m_{l}}\right)=P\left(X_{m_{l-1}+1} \leq x_{m_{l-1}+1}, \ldots X_{m_{l}} \leq x_{m_{l}}\right)
$$

e sua cópula associada

$$
C_{H_{l}}\left(F_{X_{m_{l-1}+1}}\left(x_{m_{l-1}+1}\right), \ldots, F_{X_{m_{l}}}\left(x_{m_{l}}\right)\right)=H_{l}\left(x_{m_{l-1}+1}, \ldots, x_{m_{l}}\right)
$$

para $l=1, \ldots, d$. Pela transformada integral da probabilidade tem-se que $U_{m_{l}}=$ $F_{X_{m_{l}}}\left(X_{m_{l}}\right) \sim U(0,1)$, para todo $i=1, \ldots, n$. Seja $\Psi_{l}=H_{l}\left(X_{m_{l-1}+1}, \ldots, X_{m_{l}}\right)=$ $C_{H_{l}}\left(U_{m_{l-1}+1}, \ldots, U_{m_{l}}\right)$, nestas condições segue que a função distribuição de $\Psi_{l}$ é dada 
por

$$
\begin{aligned}
K_{l}\left(\psi_{l}\right) & =P\left(\Psi_{l} \leq \psi_{l}\right) \\
& =P\left(H_{l}\left(X_{m_{l-1}+1}, \ldots, X_{m_{l}}\right) \leq \psi_{l}\right) \\
& =P\left(C_{H_{l}}\left(U_{m_{l-1}+1}, \ldots, U_{m_{l}}\right) \leq \psi_{l}\right), \quad \psi_{l} \in[0,1],
\end{aligned}
$$

em que $K_{l}\left(\psi_{l}\right)$ é nestas condições contínua e é chamada de função distribuição de Kendall, veja Nelsen et al. (2003). Portanto, $W_{l}=K_{l}\left(\Psi_{l}\right) \sim U(0,1)$ para $l=$ $1, \ldots, d$.

A distribuição de Kendall $K_{l}$ é função somente da cópula $C_{H_{l}}$ associada a $H_{l}$ e portanto, $K_{l}$ é um resumo unidimensional da estrutura de dependência dada por $C_{H_{l}}$. Note que se $n_{l}=1$ para algum $l=1, \ldots, d$, tem-se

$$
K_{l}\left(\psi_{l}\right)=P\left(\Psi_{l} \leq \psi_{l}\right)=P\left(U_{m_{l-1}+1} \leq \psi_{l}\right)=\psi_{l}, \quad \psi_{l} \in[0,1]
$$

Como $K_{l}$ 's são contínuos, de acordo com o Teorema de Sklar existe uma única cópula $d$-dimensional $C_{d}$ que é a função distribuição do vetor $\left(W_{1}, \ldots, W_{d}\right)$, i.e.

$$
C_{d}\left(w_{1}, \ldots, w_{d}\right)=P\left(W_{1} \leq w_{1}, \ldots, W_{d} \leq w_{d}\right)
$$

para $\left(w_{1}, \ldots, w_{d}\right) \in[0,1]^{d}$. Com base nestes resultados a seguinte proposição é válida.

Proposição 5.1. Sejam $\bar{X}_{1}, \ldots, \bar{X}_{d}$, d vetores aleatórios, com $H_{1}, \ldots, H_{d}$ suas funções distribuição conjunta. Seja $C_{H_{1}}, \ldots, C_{H_{d}}$ as suas cópulas correspondentes e $K_{1}\left(\psi_{1}\right), \ldots, K_{d}\left(\psi_{d}\right)$ suas distribuições de Kendall associadas. Seja $G$ a função distribuição conjunta de $\left(\Psi_{1}, \ldots, \Psi_{d}\right)$. Então existe uma única cópula d-dimensional $C_{\Psi_{1} \ldots, \Psi_{d}}$, que fornece a estrutura de dependência entre os aglomerados $\bar{X}_{1}, \ldots, \bar{X}_{d}$. 
Para ilustrar a Proposição 5.1 consideremos três exemplos. Para todos eles, considere o vetor aleatório $\left(X_{1}, X_{2}, X_{3}, X_{4}\right)$, com marginais bivariadas conhecidas $H_{1}\left(x_{1}, x_{2}\right)=P\left(X_{1} \leq x_{1}, X_{2} \leq x_{2}\right)$ e $H_{2}\left(x_{3}, x_{4}\right)=P\left(X_{3} \leq x_{3}, X_{4} \leq x_{4}\right)$ e cópulas associadas $C_{1}$ e $C_{2}$ respectivamente. Seja $G$ a distribuição conjunta das variáveis aleatórias $\Psi_{1}=C_{H_{1}}\left(U_{1}, U_{2}\right)$ e $\Psi_{2}=C_{H_{2}}\left(U_{3}, U_{4}\right)$, i.e. $G\left(\psi_{1}, \psi_{2}\right)=P\left(\Psi_{1} \leq \psi_{1}, \Psi_{2} \leq\right.$ $\psi_{2}$ ) e $C_{G}$ sua cópula associada.

Exemplo 5.3. Seja $C_{H_{1}}\left(u_{1}, u_{2}\right)=\min \left(u_{1}, u_{2}\right)$ e $C_{H_{2}}\left(u_{3}, u_{4}\right)=\min \left(u_{3}, u_{4}\right)$. Seja

$$
\left.C_{G}\left(v_{1}, v_{2}\right)\right)=v_{1} v_{2}+\theta v_{1} v_{2}\left(1-v_{1}\right)\left(1-v_{2}\right)
$$

a cópula associada a estrutura de dependência entre os aglomerados $\left(X_{1}, X_{2}\right)$ e $\left(X_{3}, X_{4}\right)$. A distribuição de Kendall associada ao vetor $\left(X_{1}, X_{2}\right)$ é:

$$
\begin{aligned}
K_{1}\left(\psi_{1}\right) & =P\left(\Psi_{1} \leq \psi_{1}\right)=P\left(C_{H_{1}}\left(U_{1}, U_{2}\right) \leq \psi_{1}\right)=P\left(\min \left(U_{1}, U_{2}\right) \leq \psi_{1}\right) \\
& =1-P\left(\min \left(U_{1}, U_{2}\right)>\psi_{1}\right)=1-P\left(U_{1}>\psi_{1}, U_{2}>\psi_{1}\right) \\
& =1-\left[1-P\left(U_{1} \leq \psi_{1}, U_{2} \leq 1\right)-P\left(U_{1} \leq 1, U_{2} \leq \psi_{1}\right)\right. \\
& \left.+P\left(U_{1} \leq \psi_{1}, U_{2} \leq \psi_{1}\right)\right] \\
& =1-\left[1-C\left(\psi_{1}, 1\right)-C\left(1, \psi_{1}\right)+C\left(\psi_{1}, \psi_{1}\right)\right] \\
& =1-\left(1-\psi_{1}-\psi_{1}+\min \left(\psi_{1}, \psi_{1}\right)\right) \\
& =1-\left(1-2 \psi_{1}+\psi_{1}\right)=\psi_{1}, \quad \psi_{1} \in[0,1]
\end{aligned}
$$

do mesmo modo temos que a distribuição de Kendall associada ao vetor $\left(X_{3}, X_{4}\right)$ é dado por $K_{2}\left(\psi_{2}\right)=\psi_{2}, \psi_{2} \in[0,1]$. Assim, a distribuiçào conjunta do vetor aleatório $\left(\Psi_{1}, \Psi_{2}\right)$ é dada por

$$
G\left(\psi_{1}, \psi_{2}\right)=C_{G}\left(K_{1}\left(\psi_{1}\right), K_{2}\left(\psi_{2}\right)\right)=\psi_{1} \psi_{2}+\theta \psi_{1} \psi_{2}\left(1-\psi_{1}\right)\left(1-\psi_{2}\right),
$$


ou equivalentemente,

$$
\begin{aligned}
G\left(C_{H_{1}}\left(u_{1}, u_{2}\right), C_{H_{2}}\left(u_{3}, u_{4}\right)\right) & =\min \left(u_{1}, u_{2}\right) \min \left(u_{3}, u_{4}\right)+\theta \min \left(u_{1}, u_{2}\right) \\
& \times \min \left(u_{3}, u_{4}\right)\left[1-\min \left(u_{1}, u_{2}\right)\right]\left[1-\min \left(u_{3}, u_{4}\right) .\right.
\end{aligned}
$$

Exemplo 5.4. Seja $C_{H_{1}}\left(u_{1}, u_{2}\right)=u_{1} u_{2}$ e $C_{H_{2}}\left(u_{3}, u_{4}\right)=u_{3} u_{4}$. A distribuição de Kendall associada ao vetor $\left(X_{1}, X_{2}\right)$ é:

$$
\begin{aligned}
K_{1}\left(\psi_{1}\right) & =P\left(\Psi_{1} \leq \psi_{1}\right)=P\left(C_{H_{1}}\left(U_{1}, U_{2}\right) \leq \psi_{1}\right)=P\left(U_{1} U_{2} \leq \psi_{1}\right) \\
& =\int_{0}^{\psi_{1}} \underbrace{P\left(U_{1} U_{2} \leq \psi_{1} \mid U_{2}=v\right)}_{=1} d v+\int_{\psi_{1}}^{1} P\left(U_{1} U_{2} \leq \psi_{1} \mid U_{2}=v\right) d v \\
& =\int_{0}^{\psi_{1}} d v+\int_{\psi_{1}}^{1} P\left(U_{1} \leq \frac{\psi_{1}}{v} \mid U_{2}=v\right) d v \\
& =\psi_{1}+\int_{\psi_{1}}^{1} \frac{\psi_{1}}{v} d v=\psi_{1}+\left.\psi_{1} \ln (v)\right|_{\psi_{1}} ^{1} \\
& =\psi_{1}-\psi_{1} \ln \left(\psi_{1}\right), \quad \psi_{1} \in[0,1]
\end{aligned}
$$

do mesmo modo temos que a distribuição de Kendall associada ao vetor $\left(X_{3}, X_{4}\right)$ é dado por $K_{2}\left(\psi_{2}\right)=\psi_{2}-\psi_{2} \ln \left(\psi_{2}\right), \psi_{2} \in[0,1]$. Se $C_{G}\left(v_{1}, v_{2}\right)=\min \left(v_{1}, v_{2}\right)$ é a cópula associada a estrutura de dependência entre os aglomerados $\left(X_{1}, X_{2}\right)$ e $\left(X_{3}, X_{4}\right)$. Deste modo, $G\left(\psi_{1}, \psi_{2}\right)=C_{G}\left(K_{1}\left(\psi_{1}\right), K_{2}\left(\psi_{2}\right)\right)=\min \left(\psi_{1}-\psi_{1} \ln \left(\psi_{1}\right), \psi_{2}-\right.$ $\left.\psi_{2} \ln \left(\psi_{2}\right)\right), o u$

$$
G\left(C_{H_{1}}\left(u_{1}, u_{2}\right), C_{H_{2}}\left(u_{3}, u_{4}\right)\right)=\min \left(u_{1} u_{2}-u_{1} u_{2} \ln \left(u_{1} u_{2}\right), u_{3} u_{4}-u_{3} u_{4} \ln \left(u_{3} u_{4}\right)\right) \text {. }
$$

Como já foi dito anteriormente, a distribıição de Kendall é 1 m resumo da estrutura de dependéncia do vetor aleatório. É claro que, quando resumimos toda informação em uma dimensão menor que a original, isto pode implicar numa perda de informação. O próximo exemplo demonstra este fato. 
Exemplo 5.5. Seja $C_{H_{1}}\left(u_{1}, u_{2}\right)=\min \left(u_{1}, u_{2}\right) e C_{H_{2}}\left(u_{3}, u_{4}\right)=\max \left(u_{3}+u_{4}-1,0\right)$. Então, a distribuição de Kendall associada a cópula $C_{H_{1}}$ é $K_{1}\left(\psi_{1}\right)=\psi_{1}, \psi_{1} \in[0,1]$, veja Exemplo 5.3 e a distribuição de Kendall associada a cópula $C_{H_{2}}$ é dada por:

$$
\begin{aligned}
K_{2}\left(\psi_{2}\right) & =P\left(\Psi_{2} \leq \psi_{2}\right)=P\left(C_{H_{2}}\left(U_{3}, U_{4}\right) \leq \psi_{2}\right)=P\left(\max \left(U_{3}+U_{4}-1,0\right) \leq \psi_{2}\right) \\
& =P\left(\max \left(U_{3}+U_{4}-1,0\right) \leq \psi_{2} \mid U_{3}+U_{4}=1\right) P\left(U_{3}+U_{4}=1\right) \\
& +P\left(\max \left(U_{3}+U_{4}-1,0\right) \leq \psi_{2} \mid U_{3}+U_{4} \neq 1\right) P\left(U_{3}+U_{4} \neq 1\right)
\end{aligned}
$$

Agora note que, para $\left(U_{3}, U_{4}\right)$ com função distribuição $C\left(u_{3}, u_{4}\right)=\max \left(u_{3}+u_{4}-1,0\right)$ tem-se que $P\left(U_{3}+U_{4}=1\right)=1$ portanto $P\left(U_{3}+U_{4} \neq 1\right)=0$, veja Nelsen (1999). Desde modo,

$$
\begin{aligned}
K_{2}\left(\psi_{2}\right) & =\underbrace{P\left(\max \left(U_{3}+U_{4}-1,0\right) \leq \psi_{2} \mid U_{3}+U_{4}=1\right)}_{=1} \underbrace{P\left(U_{3}+U_{4}=1\right)}_{=1} \\
& +P\left(\max \left(U_{3}+U_{4}-1,0\right) \leq \psi_{2} \mid U_{3}+U_{4} \neq 1\right) \underbrace{P\left(U_{3}+U_{4} \neq 1\right)}_{=0} \\
& =1
\end{aligned}
$$

Se

$$
C_{G}\left(v_{1}, v_{2}\right)=v_{1} v_{2}+\theta v_{1} v_{2}\left(1-v_{1}\right)\left(1-v_{2}\right)
$$

é a cópula associada a estrutura de dependência entre os vetores aleatórios $\left(X_{1}, X_{2}\right)$ $e\left(X_{3}, X_{4}\right)$, então

$$
G\left(v_{1}, v_{2}\right)=C_{G}\left(K_{1}\left(\psi_{1}\right) \cdot K_{2}\left(\psi_{2}\right)\right)=\iota_{1}+\theta v_{1}\left(1-v_{1}\right)(1-1)=v_{1}
$$

ou equivalentemente,

$$
G\left(C_{H_{1}}\left(u_{1}, u_{2}\right), C_{H_{2}}\left(u_{3}, u_{4}\right)\right)=\min \left(u_{1}, u_{2}\right)
$$


Como pode-se notar, a distribuição de Kendall associada a cópula $C_{H_{2}}\left(u_{3}, u_{4}\right)=$ $\max \left(u_{3}+u_{4}-1,0\right)$ é uma distribuição degenerada, isto implica neste caso que foi perdida toda a informação sobre a estrutura de dependència de $\left(X_{3}, X_{4}\right)$. Portanto, a função distribuição conjunta do vetor $\left(\Psi_{1}, \Psi_{2}\right)$ é função somente de $\psi_{1}$ e conseqüentemente depende somente de $u_{1}$ e $u_{2}$.

Na Figura 5.1 mostramos a função distribuição conjunta $G\left(C\left(u_{1}, u_{2}\right), u_{3}\right)$ com cópula associada $C(u, v)=\frac{u v}{u+v-u v}$ do vetor aleatório $\left(U_{1}, U_{2}\right)$ com a variável aleatória $U_{3}$ com $U_{i} \sim U(0,1) i=1,2,3$, considerando primeiro que $\left(U_{1}, U_{2}\right)$ tem função distribuição $C_{1}\left(u_{1}, u_{2}\right)=\min \left(u_{1}, u_{2}\right)$ e depois $C_{2}\left(u_{1}, u_{2}\right)=u_{1} u_{2}$ desta forma a distribuição de Kendall associada a $C_{1}$ é $K_{1}\left(\psi_{1}\right)=\psi_{1}$ e a $C_{2}$ é $K_{2}\left(\psi_{2}\right)=\psi_{2}-\psi_{2} \ln \left(\psi_{2}\right)$, em que $\Psi_{1}=\min \left(U_{1}, U_{2}\right)$ e $\Psi=U_{1} U_{2}$, veja Nelsen et al. (2001). Deste modo, a distribuição conjunta do vetor aleatório $\left(\Psi_{1}, U_{3}\right)$ é

$$
\begin{aligned}
G_{1}\left(\psi_{1}, u_{3}\right) & =C\left(K_{1}\left(\psi_{1}\right), u_{3}\right) \\
& =\frac{\psi_{1} u_{3}}{\psi_{1}+u_{3}-\psi_{1} u_{3}} \\
& =\frac{\min \left(u_{1}, u_{2}\right) u_{3}}{\min \left(u_{1}, u_{2}\right)+u_{3}-\min \left(u_{1}, u_{2}\right) u_{3}}
\end{aligned}
$$

do mesmo modo, para o vetor aleatório $\left(\Psi_{2}, U_{3}\right)$ tem-se

$$
\begin{aligned}
G_{2}\left(\psi_{2}, u_{3}\right) & =C\left(K_{2}\left(\psi_{2}\right), u_{3}\right) \\
& =\frac{\psi_{2}\left(1-\ln \left(\psi_{2}\right)\right) u_{3}}{\psi_{2}\left(1-\ln \left(\psi_{2}\right)\right)+u_{3}-\psi_{1}\left(1-\ln \left(\psi_{2}\right)\right) u_{3}} \\
& =\frac{u_{1} u_{2} u_{3}\left(1-\ln \left(u_{1} u_{2}\right)\right)}{u_{1} u_{2}\left(1-\ln \left(u_{1} u_{2}\right)\right)+u_{3}-u_{1} u_{2} u_{3}\left(1-\ln \left(u_{1} u_{2}\right)\right)}
\end{aligned}
$$

A metodologia apresentada nesta seção fornece ferramentas para que possamos de uma maneira simples estudar a estrutura de dependencia entre vetores aleatórios. Por exemplo, dado que conhecemos a estrutura de dependencia das marginais representadas pelas suas respectivas cópulas $C_{H_{1}}, \ldots, C_{H_{d}}$ então dada uma amostra de 

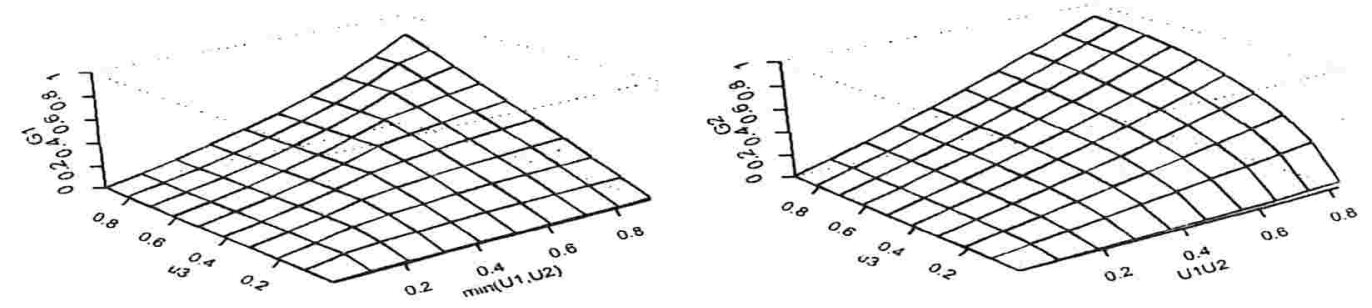

Figura 5.1: Comparação entre as funções distribuição conjunta de $\left(\Psi_{1}, U_{3}\right)$ e $\left(\Psi_{2}, U_{3}\right)$ com mesma cópula associada.

tamanho $m$ do vetor aleatório $\left(X_{1}, \ldots, X_{n}\right)$ pode-se determinar $\left\{\left(\psi_{11}, \ldots, \psi_{d 1}\right), \ldots\right.$, $\left.\left(\psi_{1 m}, \ldots, \psi_{d m}\right)\right\}$ e então utilizar alguma metodologia não paramétrica para determinar as distribuições de Kendall associadas aos vetores $K_{1}, \ldots, K_{d}$ para depois utilizando as metodologias de cópulas estudar a estrutura de dependència entre os vetores.

Neste capítulo apresentamos duas ferramentas que podem ser utilizadas para se estudar a estrutura de dependència entre vetores aleatórios. Estes trabalhos também podem ser encontrados em Anjos et al. (2004a), Anjos e Kolev (2005a) e Anjos e Kolev (2005c). 


\section{Conclusões}

Neste trabalho apresentamos alguns resultados na teoria de cópulas que poderão contribuir para novos desenvolvimentos da área. Em particular, no Capítulo 3 foi apresentado uma representação para cópulas em que a estrutura de dependência agora é condensada em uma função que denominamos função Spearman $\rho_{C}$ associada a cópula $C$ que é uma medida de dependència local "normalizada" i.e., $\rho_{C}:[0,1]^{2} \mapsto$ $[-1,1]$. A relação (3.3) na Proposição 3.1 pode ser vista como uma alternativa bivariada ao Teorema de Sklar, pois afirma de maneira análoga que para toda função distribuição conjunta $H$ existe uma função Spearman $\rho_{H}$ associada e que portanto permite a sua respectiva caracterização. Na Seção 3.3 exibimos uma medida de dependência local $r(u, v)$ que permite uma associação "local" entre uma cópula $C$ e uma cópula gaussiana $\Phi_{r}$. Este resultado pode ser útil na formulação de uma família de cópulas cuja a especificação das estruturas de dependência bivariadas especificam totalmente a cópula $n$-variada, $n \geq 2$. Na prática isto implica que se por exemplo tivermos $\left(X_{1}, X_{2}, X_{3}\right)$ poderemos especificar as cópulas bivariadas $C_{X_{1}, X_{2}}, C_{X_{1} . X_{3}}, C_{X_{2}, X_{3}}$ e a cópula $C_{X_{1}, X_{2}, X_{3}}$ estará automaticamente determinada.

De 1 m modo geral para $\left(X_{1}, \ldots, X_{n}\right)$ tem-se o seguinte:

$$
C_{X_{1} \ldots, Y_{n}}\left(u_{1}, \ldots, u_{n}\right)=\Phi_{\mathbf{r}}\left(\Phi^{-1}\left(u_{1}\right), \ldots, \Phi^{-1}\left(u_{n}\right)\right)
$$


em que

$$
\mathbf{r}=\left(\begin{array}{cccc}
1 & r_{X_{1}, X_{2}}\left(u_{1}, u_{2}\right) & \cdots & r_{X_{1}, X_{n}}\left(u_{1}, u_{n}\right) \\
r_{X_{2}, X_{1}}\left(u_{2}, u_{1}\right) & 1 & \cdots & r_{X_{2}, X_{n}}\left(u_{2}, u_{n}\right) \\
\vdots & \vdots & \ddots & \vdots \\
r_{X_{n}, X_{1}}\left(u_{n}, u_{1}\right) & r_{X_{n}, X_{2}}\left(u_{n}, u_{2}\right) & \cdots & 1
\end{array}\right)
$$

em que $r_{X_{i}, X_{j}}\left(u_{i}, u_{j}\right)=r_{X_{j}, X_{i}}\left(u_{j}, u_{i}\right)=\inf \left\{r: \Phi_{r}\left(\Phi^{-1}\left(u_{i}\right), \Phi^{-1}\left(u_{j}\right)\right) \geq C_{X_{i}, X_{j}}\left(u_{i}, u_{j}\right)\right\}$ e $C_{X_{i}, X_{j}}$ é a estrutura de dependència entre $X_{i}$ e $X_{j}$. Note que a cópula gaussiana é um caso particular desta família quando temos $\mathbf{r}$ constante para todo $\left(u_{1}, \ldots, u_{n}\right) \in$ $[0,1]^{n}$. O problema nesta definição é determinar quais são as condições que devemos ter sobre as cópulas bivariadas $C_{X_{i}, X_{j}}$ para que $C_{X_{1}, \ldots, X_{n}}$ seja uma cópula. além da restrição óbvia que $\mathbf{r}$ deve ser uma matriz positiva definida. Todos os resultados deste capítulo também podem ser encontrados nos seguintes artigos: Anjos e Kolev (2005b) e Kolev et al. (2005).

No Capítulo 4 apresentamos resultados relacionados com as estatísticas de ordem tais como cópula associada, relações de recorrência que podem ser utilizadas para calcular momentos associados assim como suas fronteiras de Fréchet associadas. Os resultados deste capítulo resultaram nos seguintes artigos: Anjos et al. (2004b) e Kolev et al. (2005).

No Capítulo 5 mostramos as dificuldades que se apresentam quando tentamos construir distribuiçōes cujas marginais especificadas são multivariadas e fornecemos duas metodologias para se estudar a dependencia entre vetores aleatórios. uma baseada numa simples adaptação do método proposto em Cohen (1984) e outra totalmente nova baseada na distribuição de Kendall associada. Estes resultados também podem ser vistos em Anjos et al. (2004a), Anjos e Kolev (2005a) e Anjos e Kolev (2005c).

Neste trabalho nảo foi tratada o problema da inferéncia. Esta é uma etapa a ser 
feita no futuro em que iremos avaliar o potencial das ferramentas apresentadas. Por exemplo, como podemos utilizar a função Spearman na escolha da melhor cópula. Um outro problema é o seguinte: Dado uma carteira de ativos, suponha que consigamos dividir os ativos em très grupo por exemplo, e embora não saibamos qual a estrutura de dependência dentro de cada grupo, sabemos qual a estrutura entre os grupos. Qual será a perda máxima esperada desta carteira? Os resultados do Capítulo 5 podem ser utilizados na determinação desta fronteira. 


\section{Referências Bibliográficas}

Anjos, U., Ferreira, F., Kolev, N., Mendes, B. (2004a). Modelando Dependências via Cópulas. Associação Brasileira de Estatística: São Paulo.

Anjos, U., Kolev, N., Tanaka, N. (2004b). Copula associated to order statistics. RT-MAE 2004-16, Universidade de São Paulo.

Anjos, U., Kolev, N. (2005a). Copulas with given nonoverlapping multivariate marginals. RT-MAE 2005-02, Universidade de São Paulo.

Anjos, U., Kolev, N. (2005b). Representation of bivariate copulas via local measure of dependence. RT-MAE 2005-03, Universidade de São Paulo. Submetido.

Anjos, U., Kolev, N. (2005c). An application of Kendall distribution. Tijdschrift voor Economie en Management L(1), 95 - 101.

Arnold, B.C., Castillo, E., Saraiba, J.M. (1999). Conditional specification of statistical model. Springer: New York.

Avérous, J., Genest, C., Kochar, S. (2005). On the dependence structure of order statistics. Journal of Multivariate Analysis 94, 159-171

Balakrishnan, N., Cohen, A.C. (1991). Order Statistics and Inference. Academic Press: San Diego. 
Barakat, H. (2001). The asymptotic distribution theory of bivariate order statistics. Ann. Inst. Stat. Math. 53, 487-497.

Bjerve, S., Doksum, K. (1993). Correlation curves: measures of association as functions of covariate values. Annals of Statistics 21, 890-902.

Blest, D.C. (2000). Rank correlation - an alternative measure. Australian and New Zealand Journal of Statistics 42, 101-110.

Cherubini, U., Luciano, E., Vecchiato, W. (2004). Copula Methods in Finance. Wiley Finance: Chichester.

Cohen, L., (1984). Probability distributions with given multivariate marginals. Journal of Mathematical Physics 25, 2402-2403.

Cuadras, C. (1992). Probability distributions with given multivariate marginals and given dependence structure. Journal of Multivariate Analysis 42, 51-66.

Dall'Aglio, G. (1972). Fréchet classes and compatibility of distribution functions. in Symposia Mathematica IX, 131-150.

David, H. (1981). Order Statistics. 2nd edition. John Wiley \& Sons: New York.

Dhaene, J., Denuit, M., Goovaerts, M.J., Kaas, R., Vyncke, D. (2002). The concept of comonotonicity in actuarial science and finance: theory. Insurance: Mathematics and Economics 31, 3-33.

Doksum, K., Blyth, S., Bradlow, E., Meng, X.L., Zhao, H. (1994). Correlation curves as local measures of variance explained by regression. Journal of the American Statistical Association 89. 571-582.

Embrechts, P., McNeil, A., Strauman, D. (2002). Correlation and dependence in risk management: properties and pitfalls. In Risk Management: Value at Risk and 
Beyond, (Dempster, M., Moffatt, H.K. (Eds.)). Cambridge Univ. Press: Cambridge, $176-223$.

Embrechts, P., Lindskog, F., McNeil, A. (2003). Modelling dependence with copulas and applications to risk management. In Handbook of Heavy Tailed distributions in Finance, (Rachev, S.T. (Ed.)), Elsevier, 329-384.

Embrechts, P., Hoeing, A., Juri, A. (2003). Using Copulae to bound the Value-atRisk for functions of dependent risks. Finance \& Stochastics 7(2), 145-167.

Feller. W. (1968). An Introduction to Probability Theory and Its Applications, Volume I, 3rd edition, John Wiley \& Sons: New York.

Galambos, J. (1975). Order statistics of sample from multivariate distributions. Journal of the American Statistical Association 70, 674-680.

Genest, C., McKay, J. (1986). The Joy of Copulas: Bivariate Distributions with Uniform Marginals. American Statistician 40, 280-283.

Genest, C., Rivest, L. (1993). Statistical inference procedures for bivariate archimedean copulas. Journal of the American Statistical Association 88, 1034-1043.

Genest, C., Quesada-Molina, J.J., Rodrigues-Lallena, J.A. (1995). De l'impossibilité de construire des lois à marges multidimensionnelles données à partir de copules. Comptes rendus de l'Académie des sciences de Paris 320, série I 723-726.

Genest, C., Plante J.-F. (2003). On Blest's measure of rank correlation. The Canadian Journal of Statistics 1, 1-18.

Georges, P., Lamy, A.-G.. Nicolas, G., Quibel, G., Roncalli, T. (2001). Multivariate survival modeling: a unified approach with copulas. Crédit Lyonnais (Working paper), available at http://gro.creditlyonnais.fr/content/wp/copula-survival.pdf. 
Joe, H. (1997). Multivariate Models and Dependence Concepts. Chapman \& Hall: London.

Jones, M.C. (1996). The local dependence function, Biometrika 83, 899-904.

Jones, M.C. (1998). Constant local dependence, Journal of Multivariate Analysis 64, 148-155.

Kellerer, H. (1964a). Masstheoretische Marginalprobleme. Math. Ann. 153, 168-198.

Kellerer, H. (1964b). Verteilungsfunctionen mit gegebenen Marginalverteilungen. Zeit. Wahrscheinlichkeitsth. 3, 247-270.

Kolev, N., Mendes, B.V.M., Anjos, U. (2005). Copulas: a Review and Recent Developments. RT-MAE 2005-07, Universidade de São Paulo. Submetido.

Kotz, S., Nadarajah, S. (2003). Local dependence functions for the elliptically symmetric distributions. Sankhyā A 65, 207-223.

Kowalczyk, T. (2000). Link between grade measure of dependence and of separability in pairs of conditional distributions. Statistics \& Probability Letters 46, 371-379.

Li, H., Scarsini, M., Shaked, M. (1996). Linkages: a tool for the construction of multivariate distributions with given nonoverlapping multivariate marginals. Journal of Multivariate Analysis 56, 20-41.

Li, H., Scarsini, M., Shaked, M. (1999). Dynamic linkages for multivariate distributions with given nonoverlapping multivariate marginals. Journal of Multivariate Analysis 68. 54-77.

Marco, J.M., Ruiz-Rivas, C. (1992). On the construction of multivariate distributions with given nonoverlapping multivariate marginals. Statistics \& Probability Letters 15, 259-265. 
Meyer R. (1969). A note on a "multivariate" form of Bonferroni's inequalities. The Annals of Mathematical Statistics 40, 692-693.

Nelsen, R. (1999). An Introduction to Copulas. Springer: New York.

Nelsen, R., Quesada-Molina, J., Rodrigues-Lallena, J., Ubeda-Flores, M. (2001). Distribution function of copulas: a class of bivariate probability integral transforms. Statistics \& Probability Letters 54, 277-282.

Nelsen, R. (2002). Concordance and copulas: A survey. In Distributions with Given Marginals and Statistical Modelling, (Cuadras, C.M., Fortiana, J., Rodríguez Lallena, J.A. (Eds.)), Kluwer Academic Publishers: Dordrecht, 169-178.

Nelsen, R., Quesada-Molina, J., Rodrigues-Lallena, J., Ubeda-Flores, M. (2003). Kendall distribution functions. Statistics \& Probability Letters 65, 263-268.

Nelsen, R. (2003). Properties and applications of copulas: a brief survey. In Proceedings of the First Brazilian Conference on Statistical Modeling in Insurance and Finance, Dhaene, J., Kolev, N., Morettin, P.A. (eds), University Press USP: São Paulo, 10-28.

Niewiadomska-Bugaj, M., Kowalczyk, T. (2005). On grade transformation and its implications for copulas. A aparecer em Brazilian Journal of Probability and Statistics.

Schweizer. R.. Sklar, E. (1983). Probabilistic Metric Spaces. Elsevier: New York.

Sklar. A. (1959). Fonctions de répartition á $n$ dimensions et leurs marges. Publ. Inst. Statist. Univ. Paris 8. 229-231.

Szczesny, W. (1991). On the performance of a discriminant function. Journal of Classification 8. 201-215. 


\section{Apêndice A}

\section{Programas}

\section{A.1 Programa Seção 4.2}

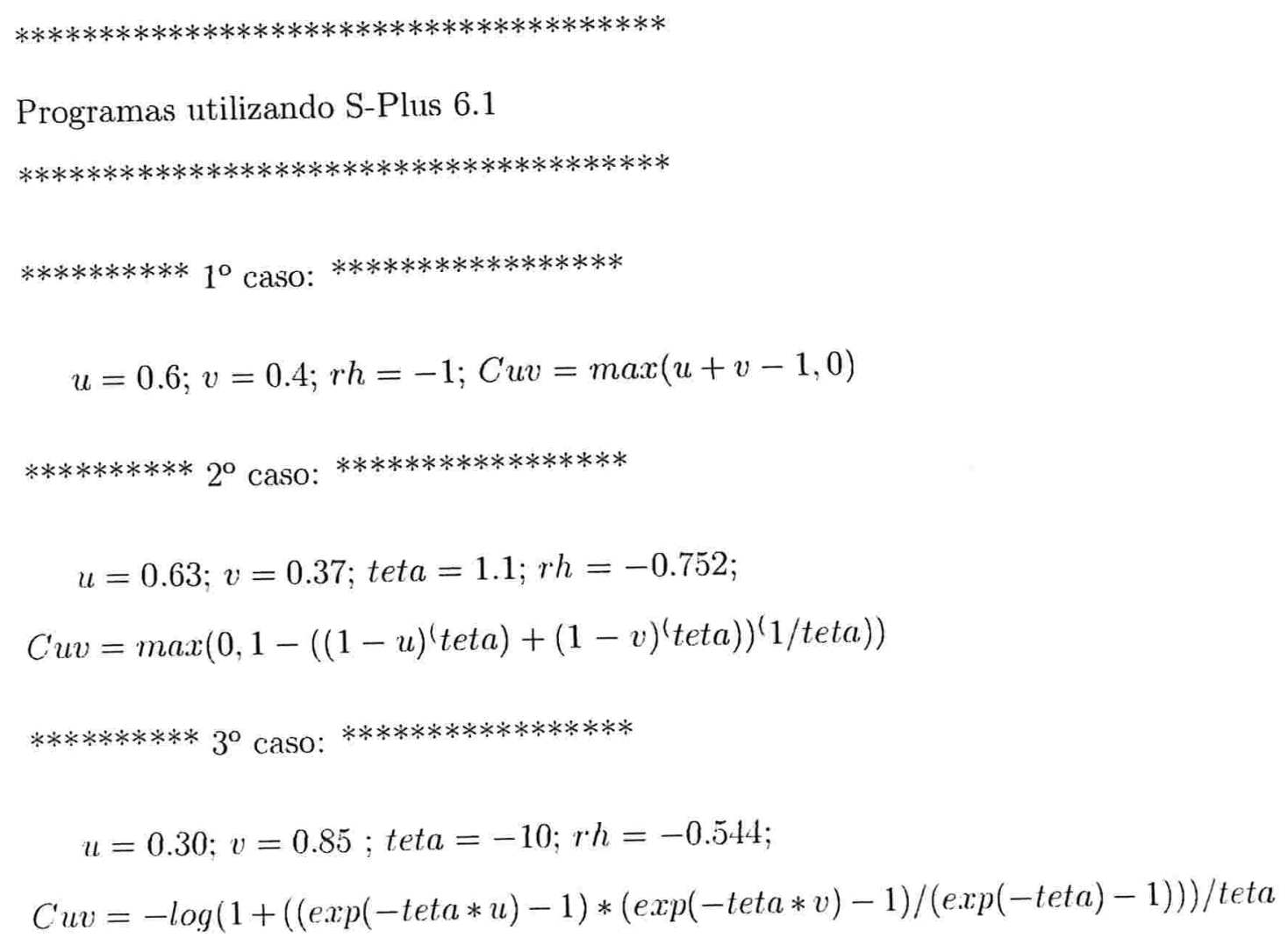




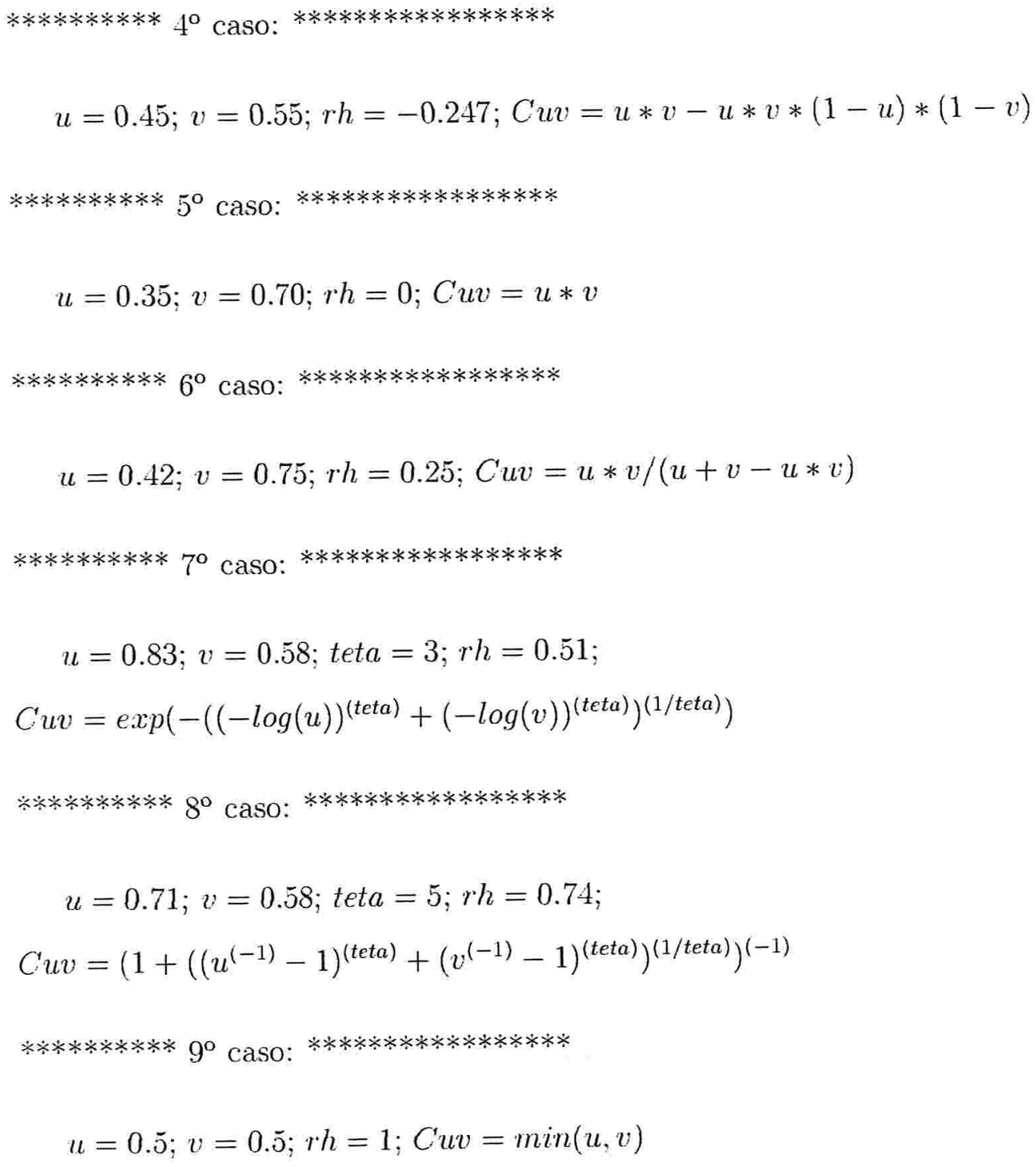

******* Cálculo usando a cópula assintótica $* * * * * * * * *$

$$
\begin{aligned}
& n=10 ; r=4 ; s=6 ; w=\operatorname{pbeta}(u, r, n-r+1) ; t=\operatorname{pbeta}(v, s, n-s+1) \\
& r h=(C u v-u * v) / \operatorname{sqrt}(u * v *(1-u) *(1-v)) \\
& \operatorname{prs} a=w+t-1+\operatorname{pmvnorm}(c(q \operatorname{morm}(1-w), \operatorname{qnorm}(1-t)), r h o=r h)
\end{aligned}
$$




\section{A.2 Programa Seção 4.4}

$\mathrm{n}=10 ; \mathrm{r}=2 ; \mathrm{s}=3 ; \mathrm{u}=\operatorname{pnorm}(-1.5) ; \mathrm{v}=\operatorname{pnorm}(-1) ; \mathrm{w}=\operatorname{pbeta}(\mathrm{u}, \mathrm{r}, \mathrm{n}-\mathrm{r}+1)$;

$\mathrm{t}=\operatorname{pbeta}(\mathrm{v}, \mathrm{s}, \mathrm{n}-\mathrm{s}+1)$

Cuv $=\operatorname{pmvnorm}(\mathrm{c}(\mathrm{qnorm}(\mathrm{u}), \mathrm{qnorm}(\mathrm{v})), \mathrm{rho}=-0.5)$

iw $=\operatorname{matrix}(0, n, n)$ it $=\operatorname{array}(c(0,0,0), \operatorname{dim}=c(n, n, n))$

Cálculo das fronteiras

$* * * * * * * * * * * * * * * * * * * * * * * * * * * * * * * * * * * * * * * * * * * * * * * * *$

$\operatorname{tauk}=0 ; \mathrm{k}=10$;

for $(\mathrm{t} 1$ in $(\mathrm{r}+\mathrm{s}):(\mathrm{r}+\mathrm{s}+\mathrm{k}))$

$\{$ for $(\mathrm{i}$ in $\mathrm{r}: \min (\mathrm{n},(\mathrm{t} 1-\mathrm{s})))$

$\{\mathrm{j}=\mathrm{t} 1-\mathrm{i}$

if $(j<=n)$

$\{\mathrm{li}=\max (0,(\mathrm{i}+\mathrm{j}-\mathrm{n})) \mathrm{ls}=\min (\mathrm{i}, \mathrm{j}) \mathrm{Wij}=0$

for (d in li:ls )

$\{$ Tijd $=(\operatorname{gamma}(\mathrm{n}+1) /(\operatorname{gamma}(\mathrm{d}+1) * \operatorname{gamma}(\mathrm{i}-\mathrm{d}+1) * \operatorname{gamma}(\mathrm{j}-\mathrm{d}+1) * \operatorname{gamma}(\mathrm{n}-$ $\mathrm{i}-\mathrm{j}+\mathrm{d}+1)))^{*} C u v^{d} * u^{(i-d)} * v^{(j-d)}$

$W i j=W i j+T i j d$

it $[\mathrm{i} . \mathrm{j}, \mathrm{d}]=$ Tijd

\}

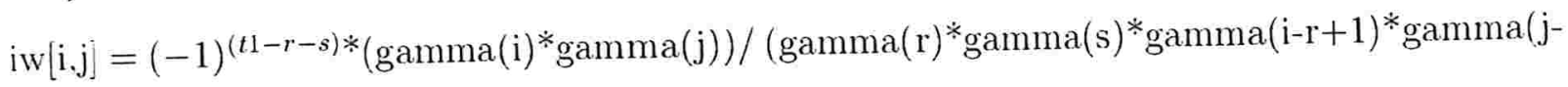


$s+1))^{*} W i j$

$\operatorname{tauk}=\operatorname{tauk}+(-1)^{(t 1-r-s) *}(\operatorname{gamma}(\mathrm{i}) * \operatorname{gamma}(\mathrm{j})) /(\operatorname{gamma}(\mathrm{r}) * \operatorname{gamma}(\mathrm{s}) * \operatorname{gamma}(\mathrm{i}-$ $\mathrm{r}+1) * \operatorname{gamma}(\mathrm{j}-\mathrm{s}+1)) * \mathrm{Wij}$

\} \} \}

Valor exato

tauk $=0$

for (i in $r: n$ )

\{

for ( $\mathrm{j}$ in $s: n)$

\{

$\mathrm{li}=\max (0,(\mathrm{i}+\mathrm{j}-\mathrm{n}))$

ls $=\min (\mathrm{i}, \mathrm{j})$

$\mathrm{Wij}=0$

for (d in li:ls )

\{

Tijd $=\left(\operatorname{gamma}(\mathrm{n}+1) /\left(\operatorname{gamma}(\mathrm{d}+1){ }^{*} \operatorname{gamma}(\mathrm{i}-\mathrm{d}+1)^{*} \operatorname{gamma}(\mathrm{j}-\mathrm{d}+1)^{*}\right.\right.$

$\operatorname{gamma}(\mathrm{n}-\mathrm{i}-\mathrm{j}+\mathrm{d}+1)))^{*} C u v^{d} *(u-C u v)^{(i-d)} *(v-C u v)^{(j-d)} *$

$(1-u-v+C u v)^{(n-i-j+d)}$

$W_{i j}=W i j+T i j d$

\}

tauk $=\operatorname{tauk}+$ Wij

\}

\} 